\title{
Diastereoselective One-Pot Synthesis of Oxazolines Using Sulfur Ylides and Acyl Imines
}

Md Shafaat Al Mehedi and Jetze J. Tepe*

Department of Chemistry, Michigan State University, East Lansing, Michigan 48823, United States

\section{Supporting Information}

\begin{tabular}{|l|c|}
\hline Table of Contents & Page 1 \\
\hline Table S1. Lewis acid optimization & S2 \\
\hline Synthesis of 1-benzotriazolyl- $N$-benzoyl-1-phenyl methylamine & S2 \\
\hline General method to synthesize substituted benzyldimethylsulfonium tetrafluoroborate salts & S3 \\
\hline Synthesis of 1-methoxy- $N$-benzoyl-1-phenyl-methylamine & S5 \\
\hline General method to synthesize tosyl containing amide & S6 \\
\hline Synthesis of Stevens rearrangement product & S10 \\
\hline General method to synthesize substituted benzyltetrahydrothiophenium tetrafluoroborate salts & S11 \\
\hline Synthesis of 1-(4-cyanobenzyl) tetrahydrothiophenium bromide salts & S12 \\
\hline Synthesis of amidine and 1,3-diaza-1,3-butadiene & $S 13$ \\
\hline${ }^{1}$ H and ${ }^{13} C$ Spectra & $S 15$ \\
\hline
\end{tabular}


Table S1. Lewis acid optimization

\begin{tabular}{|c|c|c|c|c|}
\hline L. A. (A) & $\begin{array}{c}(\mathrm{X}) \\
\text { S. Ylides }\end{array}$ & $\mathrm{DBU}(\mathrm{Y})$ & $\begin{array}{c}\text { \% Yield } \\
\text { (Mixture) }\end{array}$ & $\begin{array}{c}\text { Trans Oxazoline: Cis Aziridine: Trans } \\
\text { Aziridine }\end{array}$ \\
\hline $\mathrm{BF}_{3} . \mathrm{OEt}_{2}(5)$ & 3.5 & 5 & 41 & $1: 0: 0$ \\
\hline $\mathrm{BF}_{3} . \mathrm{OEt}_{2}(2)$ & 3.5 & 5 & 84 & $2.4: 0.8: 1$ \\
\hline $\mathrm{BF}_{3} . \mathrm{OEt}_{2}(10)$ & 3.5 & 5 & 51 & 1:0:0 \\
\hline $\mathrm{BF}_{3} \cdot \mathrm{OEt}_{2}(10)$ & 5 & 10 & 33 & 1:0:0 \\
\hline $\mathrm{BF}_{3} . \mathrm{OEt}_{2}(3)$ & 1.5 & 3 & 60 & 1:0:0 \\
\hline $\mathrm{CuCl}_{2}(5)$ & 3.5 & 5 & 70 & 1:0.3:0.03 (2days) \\
\hline $\mathrm{ZnCl}_{2}(1)$ & 5 & 5 & 45 & 2.2:0.6:1 \\
\hline $\mathrm{AlCl}_{3}(5)$ & 3.5 & 5 & 56 & 2.3:0.5:1 \\
\hline TMSCl (5) & 3.5 & 5 & 57 & $3.2: 0.7: 1$ \\
\hline $\mathrm{TiCl}_{4}(5)$ & 3.5 & 5 & 52 & 1:0.15:0.02 \\
\hline
\end{tabular}

Synthesis of 1-benzotriazolyl-N-benzoyl-1-phenyl methylamine $(\mathbf{1})^{1-3}$<smiles>O=C(NC(c1ccccc1)n1nnc2ccccc21)c1ccccc1</smiles>

To a solution of dry toluene $(40 \mathrm{~mL})$ in a $100 \mathrm{~mL}$ dry round bottom flask was added benzotriazole ( $5.45 \mathrm{~g}, 0.05 \mathrm{~mol})$, followed by benzaldehyde ( $5.31 \mathrm{~g}, 0.05 \mathrm{~mol}$ ) and benzamide $(6.05 \mathrm{~g}, 0.05 \mathrm{~mol})$. The solution was refluxed for 24 hours using a Dean-Stark apparatus. After that, the reaction mixture was cooled to room temperature and the resulting white solid was filtered off. The solid was washed with diethyl ether and subsequently toluene to give 1benzotriazolyl- $N$-benzoyl-1-phenyl-methylamine as a white solid (13.6gm, 83\%). Melting point: $163-167{ }^{\circ} \mathrm{C}$. IR: 3329, 3280, 3062, 2963, 1651, 1636, 1517, 1487, $1337 \mathrm{~cm}^{-1} .{ }^{1} \mathrm{H}$ NMR (500 MHz, DMSO-d $\left.)\right) \delta 10.30(\mathrm{~d}, J=8.2 \mathrm{~Hz}$, 1H), 8.26 (d, $J=8.2 \mathrm{~Hz}, 1 \mathrm{H}$ ), 8.11 (d, $J=8.4 \mathrm{~Hz}, 1 \mathrm{H}$ ), $8.02-7.97$ (m, 3H), 7.55 (d, $J=7.5 \mathrm{~Hz}, 2 \mathrm{H}), 7.48$ (d, $J=7.8$ $\mathrm{Hz}, 2 \mathrm{H}), 7.44-7.38$ (m, 6H). ${ }^{13} \mathrm{C}\left\{{ }^{1} \mathrm{H}\right\}$ NMR (126 MHz, DMSO-d $\left.{ }_{6}\right) \delta 167.4,145.8,136.7,133.4,132.6,132.5,129.3$, 129.1, 128.8, 128.5, 128.1, 127.5, 124.7, 119.8, 111.7, 66.6. HRMS (ESI-TOF) m/z: [M-Benzotriazole] calcd for $\left(\mathrm{C}_{14} \mathrm{H}_{12} \mathrm{NO}^{+}\right)$210.0913; Found 210.0917. 
General method to synthesize substituted benzyldimethylsulfonium tetrafluoroborate salts (2)

To a solution of dry dichloromethane $(30 \mathrm{~mL})$ in a $100 \mathrm{~mL}$ dry round bottom flask was added the appropriate alcohol (1 equiv, $10 \mathrm{mmol}$ ) followed by dimethyl sulfide (2 equiv, $20 \mathrm{mmol}$ ) and placed under nitrogen gas. A solution of tetrafluoroboric acid (tetrafluoroboric acid diethyl ether complex) (1 equiv, $10 \mathrm{mmol}$ ) was added dropwise for 5 minutes at $0{ }^{\circ} \mathrm{C}$. The mixture was stirred for 12 hours at room temperature. Evaporation of solvent under reduced pressure gave a colorless, thick oil which was precipitated with diethyl ether to obtain the desired salt as a solid.

2a

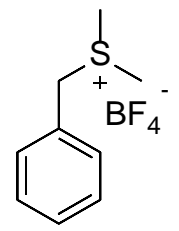

Colorless solid (2.28g, 95\%). Melting point: $101-103{ }^{\circ} \mathrm{C}$ (Lit. value 103-104 $\left.{ }^{\circ} \mathrm{C}\right){ }^{4}$ IR: 3029, 2946, 1433, 1027, 1008 $\mathrm{cm}^{-1} .{ }^{1} \mathrm{H}$ NMR (500 MHz, DMSO-d 6 ) $\delta 7.53-7.42$ (m, 5H), $4.62(\mathrm{~s}, 2 \mathrm{H}), 2.77$ (s, 6H). ${ }^{13} \mathrm{C}\left\{{ }^{1} \mathrm{H}\right\}$ NMR (126 MHz, DMSO-d $\left.{ }_{6}\right) \delta 131.1,130.1,129.8,128.7,46.2,24.2$. HRMS (ESI-TOF) m/z: [M-BF ${ }_{4}^{-}$] calcd for $\left(\mathrm{C}_{9} \mathrm{H}_{13} \mathrm{~S}^{+}\right) 153.0732$; Found 153.0745.

2b

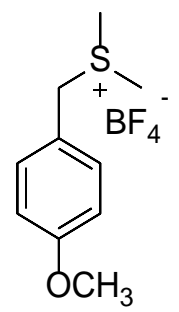

Colorless solid (2.67g, 99\%). Melting point: 58-59 ${ }^{\circ} \mathrm{C}$. IR: 3030, 2975, 1434, 1216, $1030 \mathrm{~cm}^{-1} .{ }^{1} \mathrm{H}$ NMR (500 MHz, DMSO- $\left.d_{6}\right) \delta 7.36(\mathrm{~d}, J=8.7 \mathrm{~Hz}, 2 \mathrm{H}), 7.06-7.00$ (d, $\left.J=8.7 \mathrm{~Hz}, 2 \mathrm{H}\right), 4.56(\mathrm{~s}, 2 \mathrm{H}), 3.77$ (s, 3H), $2.72(\mathrm{~s}, 6 \mathrm{H}) .{ }^{13} \mathrm{C}\left\{{ }^{1} \mathrm{H}\right\}$ NMR $\left(126 \mathrm{MHz}\right.$, DMSO- $\left.d_{6}\right) \delta 160.6,132.6,120.0,115.2,55.7,45.9,23.9$. HRMS (ESI-TOF) m/z: [M-BF ${ }_{4}^{-}$ $\left.\mathrm{S}\left(\mathrm{CH}_{3}\right)_{2}\right]$ calcd for $\left(\mathrm{C}_{8} \mathrm{H}_{9} \mathrm{O}^{+}\right)$121.0648; Found 121.0654.

2c<smiles>C[SH](C)Cc1ccc([N+](=O)[O-])cc1</smiles> 
Yellow solid (0.43g, 15\%). Melting point: $135-137^{\circ} \mathrm{C}$. IR: 3031, 2943, 1525, 1354, $1026 \mathrm{~cm}^{-1} .{ }^{1} \mathrm{H}$ NMR (500 MHz, DMSO- $\left.d_{6}\right) \delta 8.32(\mathrm{~d}, J=8.6 \mathrm{~Hz}, 2 \mathrm{H}), 7.72(\mathrm{~d}, J=8.6 \mathrm{~Hz}, 2 \mathrm{H}), 4.75(\mathrm{~s}, 2 \mathrm{H}), 2.81(\mathrm{~s}, 6 \mathrm{H}) .{ }^{13} \mathrm{C}\left\{{ }^{1} \mathrm{H}\right\} \mathrm{NMR}(126 \mathrm{MHz}$, DMSO- $\left.d_{6}\right) \delta 148.6,136.4,132.6,124.7,45.2,24.5$. HRMS (ESI-TOF) $\mathrm{m} / \mathrm{z}:\left[\mathrm{M}-\mathrm{BF}_{4}{ }^{-}\right]$calcd for $\left(\mathrm{C}_{9} \mathrm{H}_{12} \mathrm{NO}_{2} \mathrm{~S}^{+}\right)$ 198.0583; Found 198.0591.

2d

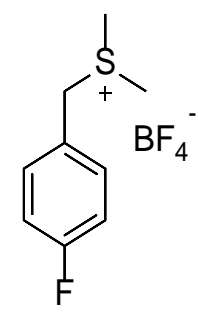

White solid (1.78g, 69\%). Melting point: $105-108{ }^{\circ} \mathrm{C}$. IR: 3033, 2942, 1512, 1226, $1026 \mathrm{~cm}^{-1} .{ }^{1} \mathrm{H}$ NMR (500 MHz, DMSO- $\left.d_{6}\right) \delta 7.50(\mathrm{dd}, J=8.8,5.4 \mathrm{~Hz}, 2 \mathrm{H}), 7.32(\mathrm{t}, J=8.8 \mathrm{~Hz}, 2 \mathrm{H}), 4.61(\mathrm{~s}, 2 \mathrm{H}), 2.76(\mathrm{~s}, 6 \mathrm{H}) .{ }^{13} \mathrm{C}\left\{{ }^{1} \mathrm{H}\right\}$ NMR $(126$ MHz, DMSO- $d_{6}$ ) $\delta 164.2$ (d, $\left.J=246 \mathrm{~Hz}\right), 133.5$ (d, $J=8 \mathrm{~Hz}$ ), 125.0 (d, $\left.J=3 \mathrm{~Hz}\right), 116.7$ (d, $J=22 \mathrm{~Hz}$ ), 45.4, 24.1. HRMS (ESI-TOF) m/z: [M-BF $4_{4}^{-}$calcd for $\left(\mathrm{C}_{9} \mathrm{H}_{12} \mathrm{FS}^{+}\right)$171.0638; Found 171.0644.

2e

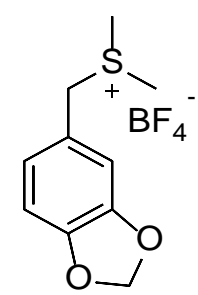

Light grey solid (2.69g, 95\%). Melting point: $115-118{ }^{\circ} \mathrm{C}$. IR: 3034, 2970, 1421, 1054, 1039, $1016 \mathrm{~cm}^{-1} .{ }^{1} \mathrm{H}$ NMR (500 MHz, DMSO-d $\left.d_{6}\right) \delta 7.06-6.98$ (m, 2H), 6.93 (dd, $\left.J=8.0,1.6 \mathrm{~Hz}, 1 \mathrm{H}\right), 6.07$ (s, 2H), $4.53(\mathrm{~s}, 2 \mathrm{H}), 2.75$ (s, 6H). ${ }^{13} \mathrm{C}\left\{{ }^{1} \mathrm{H}\right\}$ NMR (126 MHz, DMSO- $d_{6}$ ) $\delta 148.9,148.3,125.3,121.7,110.9,109.4,102.1,46.4,24.0$. HRMS (ESI-TOF) m/z: $\left[\mathrm{M}-\mathrm{BF}_{4}{ }^{-}-\mathrm{S}\left(\mathrm{CH}_{3}\right)_{2}\right]$ calcd for $\left(\mathrm{C}_{8} \mathrm{H}_{7} \mathrm{O}_{2}{ }^{+}\right)$135.0441; Found 135.0448.

2f

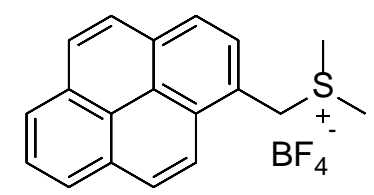

Greenish brown solid (3.28g, 90\%). Melting point: 135-139 ${ }^{\circ} \mathrm{C}$. IR: 3038, 2964, 1603, 1421, $1039 \mathrm{~cm}^{-1} .{ }^{1} \mathrm{H}$ NMR (500 MHz, DMSO- $\left.d_{6}\right) \delta 8.65$ (dd, $J=9.3,2.8 \mathrm{~Hz}, 1 \mathrm{H}$ ), 8.39 (ddd, $J=11.6,9.3,4.3 \mathrm{~Hz}, 4 \mathrm{H}$ ), 8.28 (dd, $J=8.9,6.4 \mathrm{~Hz}, 1 \mathrm{H}$ ), 8.25 - 8.21 (m, 1H), 8.19 - 8.12 (m, 2H), 5.42 (s, 2H), 2.90 (s, 6H). ${ }^{13} \mathrm{C}\left\{{ }^{1} \mathrm{H}\right\}$ NMR (126 MHz, DMSO-d 6 ) $\delta 132.5$, 131.1, 130.6, 130.4, 130.3, 129.3, 129.0, 127.7, 127.3, 126.7, 126.5, 125.6, 124.7, 124.0, 123.3, 122.1, 44.8, 24.8. HRMS (ESI-TOF) m/z: [M-BF $\left.{ }^{-}-\mathrm{S}\left(\mathrm{CH}_{3}\right)_{2}\right]$ calcd for $\left(\mathrm{C}_{17} \mathrm{H}_{11}{ }^{+}\right)$215.0861; Found 215.0865. 


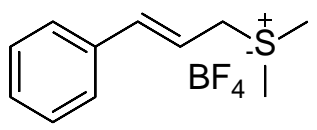

Brownish solid (2.45g, 92\%). Melting point: 48-52 ${ }^{\circ} \mathrm{C}$. IR: 3035, 2942, 1650, 1428, 1047, $1022 \mathrm{~cm}^{-1} .{ }^{1} \mathrm{H}$ NMR (500 MHz, DMSO-d $\left.{ }_{6}\right) \delta 7.55$ (d, $\left.J=7.3 \mathrm{~Hz}, 2 \mathrm{H}\right), 7.38(\mathrm{t}, J=7.3 \mathrm{~Hz}, 2 \mathrm{H}), 7.32(\mathrm{t}, J=7.3 \mathrm{~Hz}, 1 \mathrm{H}), 6.86(\mathrm{~d}, J=15.6 \mathrm{~Hz}$, 1H), 6.34 (dt, $J=15.6,7.7 \mathrm{~Hz}, 1 \mathrm{H}), 4.18$ (d, $J=7.7 \mathrm{~Hz}, 2 \mathrm{H}), 2.82(\mathrm{~s}, 6 \mathrm{H}) .{ }^{13} \mathrm{C}\left\{{ }^{1} \mathrm{H}\right\}$ NMR $(126 \mathrm{MHz}$, DMSO-d 6 ) $\delta$ 140.6, 135.7, 129.3, 129.2, 127.5, 115.7, 44.4, 23.6. HRMS (ESI-TOF) m/z: [M-BF $\left.{ }_{4}^{-}-\mathrm{S}\left(\mathrm{CH}_{3}\right)_{2}\right]$ calcd for $\left(\mathrm{C}_{9} \mathrm{H}_{9}{ }^{+}\right)$ 117.0699; Found 117.0705.

2h

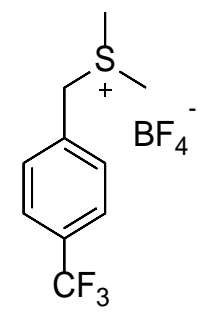

White solid (1.93g, 63\%). Melting point: $112-116{ }^{\circ} \mathrm{C}$. IR: 3028, 2941, 1423, 1323, $1022 \mathrm{~cm}^{-1} .{ }^{1} \mathrm{H}$ NMR (500 MHz, DMSO-d $\left.d_{6}\right) \delta 7.86(\mathrm{~d}, J=8.0 \mathrm{~Hz}, 2 \mathrm{H}), 7.67$ (d, $\left.J=8.0 \mathrm{~Hz}, 2 \mathrm{H}\right), 4.70$ (s, 2H), 2.81 (s, 6H). ${ }^{13} \mathrm{C}\left\{{ }^{1} \mathrm{H}\right\} \mathrm{NMR}(126 \mathrm{MHz}$, DMSO- $\left.d_{6}\right) \delta 133.6,132.0,130.2$ (q, $J=32 \mathrm{~Hz}$ ), 126.7 (q, $\left.J=3.5 \mathrm{~Hz}\right), 123.3$, 45.4, 24.4. HRMS (ESI-TOF) m/z: [M$\left.\mathrm{BF}_{4}^{-}\right]$calcd for $\left(\mathrm{C}_{10} \mathrm{H}_{12} \mathrm{~F}_{3} \mathrm{~S}^{+}\right)$221.0606; Found 221.0616.

Synthesis of 1-methoxy-N-benzoyl-1-phenyl-methylamine (4) ${ }^{1}$<smiles>COC(NC(=O)c1ccccc1)c1ccccc1</smiles>

1-Benzotriazolyl-N-benzoyl-1-phenyl-methylamine (2.47 g, $7.54 \mathrm{mmol})$ was added in one portion to a solution of sodium methoxide in methanol $(9.04 \mathrm{mmol})$ at room temperature. The mixture was stirred at room temperature for 12 hours and poured into water $(80 \mathrm{~mL})$. The resulting precipitate was collected by filtration and dried to give pure 1methoxy- $N$-benzoyl-1-phenyl-methylamine as an off-white solid (1.2g, $66 \%)$. Melting point: $96-97^{\circ} \mathrm{C}$. IR: 3290 , 3031, 2942, 1650, $1088 \mathrm{~cm}^{-1} .{ }^{1} \mathrm{H}$ NMR (500 MHz, DMSO-d $\left.d_{6}\right) \delta 9.17$ (d, $J=9.0 \mathrm{~Hz}, 1 \mathrm{H}$ ), 7.97 - 7.91 (m, 2H), 7.56 7.52 (m, 1H), 7.50 - 7.44 (m, 4H), 7.37 (t, $J=7.4 \mathrm{~Hz}, 2 \mathrm{H}$ ), 7.32 (dd, $J=8.3,6.2 \mathrm{~Hz}, 1 \mathrm{H}), 6.25$ (d, $J=9.0 \mathrm{~Hz}, 1 \mathrm{H}$ ), 3.38 (s, 3H). ${ }^{13} \mathrm{C}\left\{{ }^{1} \mathrm{H}\right\}$ NMR (126 MHz, DMSO- $\left.d_{6}\right) \delta 167.4,140.2,134.1,132.1,128.8,128.6,128.5,128.1,126.8$, 82.3, 55.7. HRMS (ESI-TOF) m/z: [M+Na+] calcd for $\left(\mathrm{C}_{15} \mathrm{H}_{15} \mathrm{NNaO}_{2}{ }^{+}\right)$264.0995; Found 264.1000. 
General method to synthesize tosyl containing amide (5)

To a solution of dry acetonitrile $(300 \mathrm{~mL})$ in a $500 \mathrm{~mL}$ dry round bottom flask was added $p$-toluenesulfinic acid, sodium salt (1.5 equiv, $25 \mathrm{mmol}$ ) and the appropriate amide (1.5 equiv, $25 \mathrm{mmol}$ ). The reaction mixture was placed under nitrogen gas. To this resulting slurry, the appropriate aldehyde (1.0 equiv, $16.7 \mathrm{mmol}$ ) was added in one portion. The mixture was cooled to $10^{\circ} \mathrm{C}$ using an ice bath. Then, chlorotrimethylsilane (TMSCl) (2.0 equiv, $33.3 \mathrm{mmol}$ ) was slowly added to the reaction mixture to maintain an internal temperature below $25{ }^{\circ} \mathrm{C}$ (addition time $15 \mathrm{~min}$ ). After complete addition of the TMSCl, the reaction was warmed to room temperature and stirred for $24 \mathrm{hrs}$. After that, water (300 mL) was added and the resulting suspension was stirred for $30 \mathrm{~min}$. The solid was isolated by filtration and the filter cake was washed with water $(100 \mathrm{~mL})$ and dried under vacuum for 24 hours to give the white powder.

$5 a$<smiles>Cc1ccc(S(=O)(=O)C(NC(=O)c2ccccc2)c2ccccc2)cc1</smiles>

Fine white solid (5.28g, 87\%). IR: 3378, 3056, 2960, 1650, 1317, $1135 \mathrm{~cm}^{-1} .{ }^{1} \mathrm{H}$ NMR (500 MHz, DMSO- $\left.d_{6}\right) \delta 9.73$ (d, $J=10.3 \mathrm{~Hz}, 1 \mathrm{H}$ ), $7.82-7.74$ (m, 4H), $7.69-7.62$ (m, 2H), $7.55-7.51$ (m, 1H), 7.43 (tt, $J=4.4,2.0 \mathrm{~Hz}, 5 \mathrm{H}), 7.38$ (d, $J=8.0 \mathrm{~Hz}, 2 \mathrm{H}), 6.60$ (d, $J=10.3 \mathrm{~Hz}, 1 \mathrm{H}), 2.35$ (s, 3H). ${ }^{13} \mathrm{C}\left\{{ }^{1} \mathrm{H}\right\}$ NMR (126 MHz, DMSO-d 6 ) $\delta 167.2,145.2$, 134.4, 133.7, 132.2, 130.9, 130.5, 130.1, 129.9, 129.5, 128.7, 128.6, 128.3, 73.1, 21.6. HRMS (ESI-TOF) m/z: $\left[\mathrm{M}+\mathrm{Na}^{+}\right]$calcd for $\left(\mathrm{C}_{21} \mathrm{H}_{19} \mathrm{NNaO}_{3} \mathrm{~S}^{+}\right)$388.0978; Found 388.0979.

$5 \mathbf{b}$

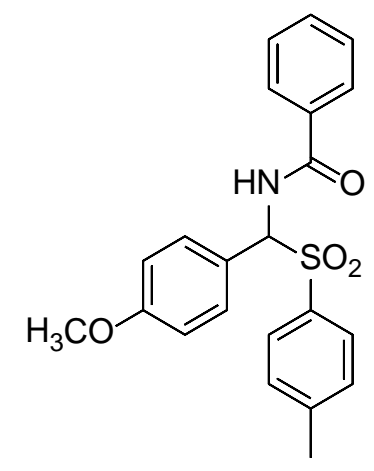

Fine white solid (5.65g, 86\%). IR: 3353, 3054, 2952, 1670, 1509, 1434, 1305, 1250, $1140 \mathrm{~cm}^{-1} .{ }^{1} \mathrm{H}$ NMR (500 MHz, DMSO-d $\left.d_{6}\right) \delta 9.65(\mathrm{~d}, J=10.3 \mathrm{~Hz}, 1 \mathrm{H}), 7.73(\mathrm{~d}, J=8.0 \mathrm{~Hz}, 2 \mathrm{H}), 7.68$ (d, $\left.J=8.8 \mathrm{~Hz}, 2 \mathrm{H}\right), 7.66-7.62(\mathrm{~m}, 2 \mathrm{H}), 7.51$ (t, $J=7.6 \mathrm{~Hz}, 1 \mathrm{H}), 7.42$ (t, $J=7.6 \mathrm{~Hz}, 2 \mathrm{H}), 7.36$ (d, $J=8.0 \mathrm{~Hz}, 2 \mathrm{H}), 6.97$ (d, $J=8.8 \mathrm{~Hz}, 2 \mathrm{H}), 6.51$ (d, $J=10.3 \mathrm{~Hz}$, 
1H), 3.76 (s, 3H), 2.34 (s, 3H). ${ }^{13} \mathrm{C}\left\{{ }^{1} \mathrm{H}\right\}$ NMR (126 MHz, DMSO-d 6 ) $\delta$ 167.1, 160.6, 145.0, 134.5, 133.7, 132.2, 131.9, 130.0, 129.4, 128.6, 128.3, 122.6, 114.1, 72.7, 55.7, 21.6. HRMS (ESI-TOF) m/z: [M-Tosyl+OCH $3+\mathrm{Na}^{+}$] calcd for $\left(\mathrm{C}_{16} \mathrm{H}_{17} \mathrm{NNaO}_{3}{ }^{+}\right)$294.1101; Found 294.1103.

$5 c$<smiles>Cc1ccc(S(=O)(=O)C(NC(=O)c2ccccc2)c2ccc([N+](=O)[O-])cc2)cc1</smiles>

Fine white solid (5.1g, 75\%). IR: 3254, 3057, 2980, 1650, 1519, 1344, 1322, 1146, $1083 \mathrm{~cm}^{-1} .{ }^{1} \mathrm{H}$ NMR (500 MHz, DMSO-d $\left.d_{6}\right) \delta .89$ (d, $\left.J=10.3 \mathrm{~Hz}, 1 \mathrm{H}\right), 8.31$ (d, $\left.J=8.8 \mathrm{~Hz}, 2 \mathrm{H}\right), 8.13(\mathrm{~d}, J=8.8 \mathrm{~Hz}, 2 \mathrm{H}), 7.82(\mathrm{~d}, J=8.2 \mathrm{~Hz}, 2 \mathrm{H})$, 7.67 (d, $J=7.4 \mathrm{~Hz}, 2 \mathrm{H}), 7.54$ (t, $J=7.4 \mathrm{~Hz}, 1 \mathrm{H}), 7.46-7.38(\mathrm{~m}, 4 \mathrm{H}), 6.87$ (d, $J=10.3 \mathrm{~Hz}, 1 \mathrm{H}) .{ }^{13} \mathrm{C}\left\{{ }^{1} \mathrm{H}\right\} \mathrm{NMR}(126$ MHz, DMSO- $\left.d_{6}\right) \delta 167.3,148.6,145.6,138.2,133.9,133.5,132.4,131.9,130.2,129.6,128.6,128.3,123.7,72.4$, 21.6. HRMS (ESI-TOF) m/z: $\left[\mathrm{M}+\mathrm{Na}^{+}\right]$calcd for $\left(\mathrm{C}_{21} \mathrm{H}_{18} \mathrm{~N}_{2} \mathrm{NaO}_{5} \mathrm{~S}^{+}\right)$433.0829; Found 433.0832 .

5d<smiles>Cc1ccc(S(=O)(=O)C(NC(=O)c2ccccc2)c2ccc(C(F)(F)F)cc2)cc1</smiles>

Fine white solid (5.61g, 78\%). IR: 3281, 3061, 2941, 1649, 1526, 1322, 1145, $1065 \mathrm{~cm}^{-1} .{ }^{1} \mathrm{H}$ NMR (500 MHz, DMSO$\left.d_{6}\right) \delta 9.84(\mathrm{~d}, J=10.3 \mathrm{~Hz}, 1 \mathrm{H}), 8.05(\mathrm{~d}, J=8.1 \mathrm{~Hz}, 2 \mathrm{H}), 7.82(\mathrm{t}, J=8.6 \mathrm{~Hz}, 4 \mathrm{H}), 7.65(\mathrm{~d}, J=8.1 \mathrm{~Hz}, 2 \mathrm{H}), 7.52(\mathrm{t}, J=$ $7.4 \mathrm{~Hz}, 1 \mathrm{H}), 7.47-7.35(\mathrm{~m}, 4 \mathrm{H}), 6.78(\mathrm{~d}, J=10.3 \mathrm{~Hz}, 1 \mathrm{H}), 2.34(\mathrm{~s}, 3 \mathrm{H}) .{ }^{13} \mathrm{C}\left\{{ }^{1} \mathrm{H}\right\}$ NMR (126 MHz, DMSO-d $d_{6} \delta$ 167.3, 145.5, 135.6, 134.1, 133.5, 132.3, 131.4, 130.3 (q, $J=31.8$ Hz), 130.1, 129.6, 128.6, 128.3, 125.6 (q, $J=3.4$ $\mathrm{Hz}), 123.4,72.5$, 21.6. HRMS (ESI-TOF) $\mathrm{m} / \mathrm{z}$ : $\left[\mathrm{M}+\mathrm{Na}^{+}\right]$calcd for $\left(\mathrm{C}_{22} \mathrm{H}_{18} \mathrm{~F}_{3} \mathrm{NNaO}_{3} \mathrm{~S}^{+}\right)$456.0852; Found 456.0855 . 
<smiles>Cc1ccc(S(=O)(=O)C(NC(=O)c2ccccc2)c2ccc(F)cc2)cc1</smiles>

Fine white solid (4.93g, 77\%). IR: 3329, 3054, 2964, 1657, 1504, 1308, $1084 \mathrm{~cm}^{-1} .{ }^{1} \mathrm{H}$ NMR (500 MHz, DMSO- $d_{6}$ ) $\delta 9.72(\mathrm{~d}, J=10.3 \mathrm{~Hz}, 1 \mathrm{H}), 7.85$ (d, $J=8.9 \mathrm{~Hz}, 2 \mathrm{H}), 7.76$ (d, $J=8.2 \mathrm{~Hz}, 2 \mathrm{H}), 7.65$ (d, $J=8.4 \mathrm{~Hz}, 2 \mathrm{H}), 7.53(\mathrm{t}, J=$ $7.4 \mathrm{~Hz}, 1 \mathrm{H}), 7.43$ (t, $J=7.4 \mathrm{~Hz}, 2 \mathrm{H}), 7.38$ (d, $J=8.2 \mathrm{~Hz}, 2 \mathrm{H}), 7.28$ (t, $J=8.9 \mathrm{~Hz}, 2 \mathrm{H}), 6.64$ (d, $J=10.3 \mathrm{~Hz}, 1 \mathrm{H}), 2.35$ (s, 3H). ${ }^{13} \mathrm{C}\left\{{ }^{1} \mathrm{H}\right\}$ NMR $(126 \mathrm{MHz}$, DMSO-d 6 ) $\delta 167.2,162.3$ (d, $J=246 \mathrm{~Hz}), 145.2,134.2,133.6,132.8$ (d, $\left.J=8 \mathrm{~Hz}\right)$, 132.3, 130.1, 129.5, 128.6, 128.3, 127.3 (d, $J=3 \mathrm{~Hz}$ ), 115.7 (d, $J=22 \mathrm{~Hz}$ ), 72.3, 21.6. HRMS (ESI-TOF) m/z: $\left[\mathrm{M}+\mathrm{Na}^{+}\right]$calcd for $\left(\mathrm{C}_{21} \mathrm{H}_{18} \mathrm{FNNaO}_{3} \mathrm{~S}^{+}\right)$406.0884; Found 406.0885.

$5 f$<smiles>Cc1ccc(S(=O)(=O)C(NC(=O)c2ccccc2)c2ccc(C#N)cc2)cc1</smiles>

Fine white solid (5.35g, 82\%). IR: 3316, 3063, 2921, 2228, 1661, 1519, 1320, $1146 \mathrm{~cm}^{-1} .{ }^{1} \mathrm{H}$ NMR (500 MHz, DMSO$\left.d_{6}\right) \delta 9.79(\mathrm{~d}, J=10.3 \mathrm{~Hz}, 1 \mathrm{H}), 8.02$ (d, $\left.J=8.3 \mathrm{~Hz}, 2 \mathrm{H}\right), 7.94$ (d, $\left.J=8.3 \mathrm{~Hz}, 2 \mathrm{H}\right), 7.79$ (d, $\left.J=8.1 \mathrm{~Hz}, 2 \mathrm{H}\right), 7.65$ (d, $J$ $=7.4 \mathrm{~Hz}, 2 \mathrm{H}), 7.53$ (t, $J=7.4 \mathrm{~Hz}, 1 \mathrm{H}), 7.43$ (t, $J=7.6 \mathrm{~Hz}, 2 \mathrm{H}), 7.39$ (d, $J=8.1 \mathrm{~Hz}, 2 \mathrm{H}), 6.79$ (d, $J=10.3 \mathrm{~Hz}, 1 \mathrm{H})$, 2.34 (s, 3H). ${ }^{13} \mathrm{C}\left\{{ }^{1} \mathrm{H}\right\}$ NMR (126 MHz, DMSO-d $\left.{ }_{6}\right) \delta 167.3,145.5,136.3,134.0,133.5,132.6,132.3,131.4,130.2$, 129.6, 128.6, 128.3, 119.0, 112.6, 72.5, 21.6. HRMS (ESI-TOF) m/z: $\left[\mathrm{M}+\mathrm{Na}+\right.$ calcd for $\left(\mathrm{C}_{22} \mathrm{H}_{18} \mathrm{~N}_{2} \mathrm{NaO}_{3} \mathrm{~S}^{+}\right)$ 413.0930; Found 413.0930. 
<smiles>COc1ccc(C(=O)NC(c2ccccc2)S(=O)(=O)c2ccc(C)cc2)cc1</smiles>

Fine white solid (6.54g, 99\%). IR: 3345, 3063, 2951, 1645, 1605, 1492, 1456, 1314, $1259 \mathrm{~cm}^{-1} .{ }^{1} \mathrm{H}$ NMR (500 MHz, DMSO-d $\left.d_{6}\right) \delta 9.51(\mathrm{~d}, J=10.3 \mathrm{~Hz}, 1 \mathrm{H}), 7.79-7.68(\mathrm{~m}, 6 \mathrm{H}), 7.41(\mathrm{~m}, 3 \mathrm{H}), 7.35$ (d, $\left.J=8.2 \mathrm{~Hz}, 2 \mathrm{H}\right), 6.96(\mathrm{~d}, J=8.8$ $\mathrm{Hz}, 2 \mathrm{H}), 6.57$ (d, $J=10.3 \mathrm{~Hz}, 1 \mathrm{H}), 3.78$ (s, 3H), $2.33(\mathrm{~s}, 3 \mathrm{H}) .{ }^{13} \mathrm{C}\left\{{ }^{1} \mathrm{H}\right\} \mathrm{NMR}\left(126 \mathrm{MHz}\right.$, DMSO-d $\left.{ }_{6}\right) \delta 166.4,162.5$, 145.1, 134.4, 131.1, 130.5, 130.3, 130.0, 129.8, 129.4, 128.6, 125.6, 113.8, 73.1, 55.9, 21.6. HRMS (ESI-TOF) m/z: $\left[\mathrm{M}-\mathrm{Tosyl}+\mathrm{OCH}_{3}+\mathrm{Na}^{+}\right]$calcd for $\left(\mathrm{C}_{16} \mathrm{H}_{17} \mathrm{NNaO}_{3}{ }^{+}\right)$294.1101; Found 294.1104.

$5 h$<smiles>Cc1ccc(S(=O)(=O)C(NC(=O)c2ccc([N+](=O)[O-])cc2)c2ccccc2)cc1</smiles>

Fine white solid (5.61g, 82\%). IR: 3309, 3061, 2963, 1667, 1514, 1308, 1141, $1084 \mathrm{~cm}^{-1} .{ }^{1} \mathrm{H}$ NMR (500 MHz, DMSO$\left.d_{6}\right) \delta 10.11(\mathrm{~d}, J=10.3 \mathrm{~Hz}, 1 \mathrm{H}), 8.28(\mathrm{~d}, J=8.7 \mathrm{~Hz}, 2 \mathrm{H}), 7.85$ (d, $\left.J=8.8 \mathrm{~Hz}, 2 \mathrm{H}\right), 7.77$ (dd, $\left.J=8.8,4.3 \mathrm{~Hz}, 4 \mathrm{H}\right)$, $7.46-7.41$ (m, 3H), 7.38 (d, $J=8.2 \mathrm{~Hz}, 2 \mathrm{H}), 6.60$ (d, $J=10.3 \mathrm{~Hz}, 1 \mathrm{H}), 2.35$ (s, 3H). ${ }^{13} \mathrm{C}\left\{{ }^{1} \mathrm{H}\right\}$ NMR (126 MHz, DMSO-d $\left._{6}\right) \delta 165.8,149.7,145.4,139.3,134.1,130.5,130.4,130.1,130.0,129.8,129.6,128.7,123.8,73.1$, 21.6. HRMS (ESI-TOF) m/z: [M+Na $]$ calcd for $\left(\mathrm{C}_{21} \mathrm{H}_{18} \mathrm{~N}_{2} \mathrm{NaO}_{5} \mathrm{~S}^{+}\right)$433.0829; Found 433.0834 . 
Synthesis of Stevens rearrangement product (6)

To a solution of dry dichloromethane $(15 \mathrm{~mL})$ in a $50 \mathrm{~mL}$ dry round bottom flask, different substituted benzyldimethylsulfonium tetrafluoroborate salts (1 equiv, $0.25 \mathrm{mmol}$ ) were added, followed by DBU base (1.5 equiv, $0.38 \mathrm{mmol}$ ) at room temperature. The reaction was placed under nitrogen gas and stirred for 12 hours. Then the solvent was evaporated, and crude products were purified using automated CombiFlash chromatography (silica gel, 20-40 microns, gradient 2-5\% ethyl acetate in hexane).

6a

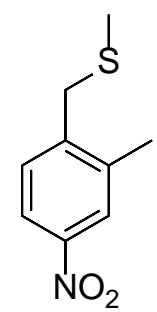

Oil (40mg, 82\%). IR: 3068, 2921, 1582, 1501, 1483, 1346, 1314, $734 \mathrm{~cm}^{-1} .{ }^{1} \mathrm{H}$ NMR $\left(500 \mathrm{MHz}, \mathrm{CHCl}_{3}-d\right) \delta 8.08-$ 7.97 (m, 2H), 7.32 (d, $J=8.3 \mathrm{~Hz}, 1 \mathrm{H}), 3.72(\mathrm{~s}, 2 \mathrm{H}), 2.48$ (s, 3H), 2.05 (s, 3H). ${ }^{13} \mathrm{C}\left\{{ }^{1} \mathrm{H}\right\} \mathrm{NMR}\left(126 \mathrm{MHz}, \mathrm{CHCl}_{3}-d\right) \delta$ 146.1, 144.8, 137.7, 131.4, 124.1, 122.2, 36.0, 19.5, 15.3. HRMS (ESI-TOF) m/z: $\left[\mathrm{M}-\mathrm{SCH}_{3}\right]$ calcd for $\left(\mathrm{C}_{8} \mathrm{H}_{8} \mathrm{NO}_{2}{ }^{+}\right)$ 150.0550; Found 150.0555.

6b

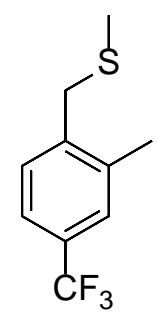

Oil (40mg, 73\%). IR: 3037, 2918, 1619, 1438, 1320, 1286, $1074 \mathrm{~cm}^{-1} .{ }^{1} \mathrm{H}$ NMR (500 MHz, $\left.\mathrm{CHCl}_{3}-d\right) \delta 7.42$ (m, 2H), 7.28 (d, $J=7.9 \mathrm{~Hz}, 1 \mathrm{H}), 3.70$ (s, 2H), 2.45 (s, 3H), 2.04 (s, 3H). ${ }^{13} \mathrm{C}\left\{{ }^{1} \mathrm{H}\right\}$ NMR (126 MHz, $\mathrm{CHCl}_{3}-d$ ) $\delta 140.9,136.8$, 131.0, 128.0 (q, $J=32.3 \mathrm{~Hz}$ ), 126.1 (q, $J=3.7 \mathrm{~Hz}$ ), 125.3, 123.9, 36.1, 19.2, 15.2. HRMS (ESI-TOF) m/z: [M-SCH calcd for $\left(\mathrm{C}_{9} \mathrm{H}_{8} \mathrm{~F}_{3}{ }^{+}\right)$173.0573; Found 173.0575.

6c

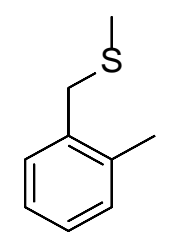

Oil (11mg, 28\%) ${ }^{6}$ (mixture with another compound). IR: 3017, 2913, 1492, 1239, $1049 \mathrm{~cm}^{-1} .{ }^{1} \mathrm{H}$ NMR (500 MHz, $\left.\mathrm{CHCl}_{3}-d\right) \delta 7.34$ (d, $\left.J=6.5 \mathrm{~Hz}, 1 \mathrm{H}\right), 7.21-7.17$ (m, 3H), 3.70 (s, 2H), $2.42(\mathrm{~s}, 3 \mathrm{H}), 2.05$ (s, 3H). ${ }^{13} \mathrm{C}\left\{{ }^{1} \mathrm{H}\right\}$ NMR $(126$ $\left.\mathrm{MHz} \mathrm{CHCl}_{3}-d\right) \delta 136.7,135.9,130.7,129.7,127.3,125.7,36.3,19.2,15.2$. HRMS (ESI-TOF) m/z: [M-SCH${ }_{3}$ calcd for $\left(\mathrm{C}_{8} \mathrm{H}_{9}{ }^{+}\right)$105.0699; Found 105.0704. 
General method to synthesize substituted benzyltetrahydrothiophenium tetrafluoroborate salts (7).

To a solution of dry dichloromethane $(30 \mathrm{~mL})$ in a $100 \mathrm{~mL}$ dry round bottom flask was added the appropriate alcohol (1 equiv, $10 \mathrm{mmol}$ ) followed by tetrahydrothiophene (1 equiv, $10 \mathrm{mmol}$ ). A solution of tetrafluoroboric acid (tetrafluoroboric acid diethyl ether complex) (1 equiv, $10 \mathrm{mmol}$ ) was added dropwise for 10 minutes at $0{ }^{\circ} \mathrm{C}$. The mixture was stirred for 12 hours at room temperature. Evaporation of solvent under reduced pressure gave a colorless, thick oil which was precipitated with diethyl ether to obtain the desired salt as a solid.

$7 \mathbf{a}$

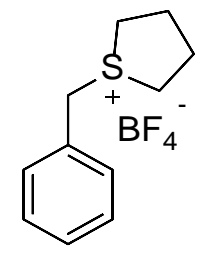

Colorless solid (2.01gm, 76\%). Melting point: $78-80{ }^{\circ} \mathrm{C}$ (Lit. value 77-79 $\left.{ }^{\circ} \mathrm{C}\right)^{7}$. IR: 3011, 2958, 1497, 1263, 1046, $1013 \mathrm{~cm}^{-1} .{ }^{1} \mathrm{H}$ NMR (500 MHz, $\mathrm{CHCl}_{3}-d$ ) $\delta 7.48-7.36$ (m, 5H), 4.54 (s, 2H), 3.54 (dt, J = 13.6, $6.6 \mathrm{~Hz}, 2 \mathrm{H}$ ), 3.42 (dt, $J=12.2,5.7 \mathrm{~Hz}, 2 \mathrm{H}), 2.29$ (qq, $J=13.6,6.6,5.7 \mathrm{~Hz}, 4 \mathrm{H}) .{ }^{13} \mathrm{C}\left\{{ }^{1} \mathrm{H}\right\} \mathrm{NMR}\left(126 \mathrm{MHz}, \mathrm{CHCl}_{3}-d\right) \delta 130.6,130.2$, 129.8, 128.0, 46.0, 42.2, 28.4. HRMS (ESI-TOF) m/z: [M-BF $\left.{ }_{4}^{-}\right]$calcd for $\left(\mathrm{C}_{11} \mathrm{H}_{15} \mathrm{~S}^{+}\right)$179.0889; Found 179.0900.

$7 b$

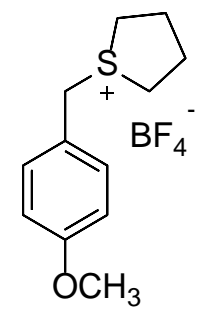

White solid (2.11, 71\%). Melting point: 96-99 ${ }^{\circ} \mathrm{C}$ (Lit. value 99-101 $\left.{ }^{\circ} \mathrm{C}\right) .{ }^{8}$ IR: 3030, 2955, 1608, 1515, 1459, 1254 , $1049 \mathrm{~cm}^{-1} .{ }^{1} \mathrm{H}$ NMR (500 MHz, DMSO-d $\left.{ }_{6}\right) \delta 7.48$ (d, $\left.J=8.6 \mathrm{~Hz}, 2 \mathrm{H}\right), 7.02$ (d, $\left.J=8.6 \mathrm{~Hz}, 2 \mathrm{H}\right), 4.47$ (s, 2H), 3.76 (s, 3H), 3.38 (m, 4H), 2.17 (m, 4H). ${ }^{13} \mathrm{C}\left\{{ }^{1} \mathrm{H}\right\}$ NMR (126 MHz, DMSO-d $\left.{ }_{6}\right) \delta 160.6,132.3,121.7,115.3,55.7,45.3,42.6$, 28.6. HRMS (ESI-TOF) m/z: [M-BF $\left.4_{4}^{-}-\mathrm{SC}_{4} \mathrm{H}_{8}\right]$ calcd for $\left(\mathrm{C}_{8} \mathrm{H}_{9} \mathrm{O}^{+}\right)$121.0648; Found 121.0655.

$7 c$

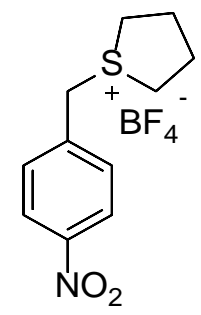

Yellow solid (1.77g, 57\%). Melting point: 101-105 ${ }^{\circ} \mathrm{C}$. IR: 3029, 2951, 1515, 1353, $1024 \mathrm{~cm}^{-1} .{ }^{1} \mathrm{H}$ NMR (500 MHz, DMSO-d $\left.d_{6}\right) \delta 8.31$ (d, $\left.J=8.5 \mathrm{~Hz}, 2 \mathrm{H}\right), 7.85$ (d, $\left.J=8.5 \mathrm{~Hz}, 2 \mathrm{H}\right), 4.65$ (s, 2H), 3.46 (m, 4H), $2.31-2.14$ (m, 4H). 
${ }^{13} \mathrm{C}\left\{{ }^{1} \mathrm{H}\right\}$ NMR $\left(126 \mathrm{MHz}\right.$, DMSO-d 6 ) $\delta$ 148.5, 138.0, 132.3, 124.8, 44.5, 43.6, 28.7. HRMS (ESI-TOF) m/z: [M-BF ${ }_{4}^{-}$ ] calcd for $\left(\mathrm{C}_{11} \mathrm{H}_{14} \mathrm{NO}_{2} \mathrm{~S}^{+}\right)$224.0745; Found 224.0747.

7d

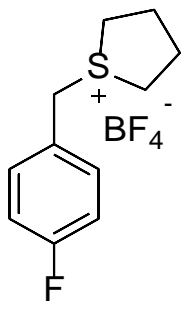

White solid (2.12g, 75\%). Melting point: $107-110{ }^{\circ} \mathrm{C}$ (Lit. value $109{ }^{\circ} \mathrm{C}$ ). ${ }^{9}$ IR: 3012, 2960, 1511, $1423,1227,1022$ $\mathrm{cm}^{-1} .{ }^{1} \mathrm{H}$ NMR (500 MHz, DMSO-d $\left.)\right) \delta .62(\mathrm{dd}, J=8.5,5.5 \mathrm{~Hz}, 2 \mathrm{H}), 7.31(\mathrm{t}, J=8.8 \mathrm{~Hz}, 2 \mathrm{H}), 4.51(\mathrm{~s}, 2 \mathrm{H}), 3.41(\mathrm{~m}$, 4H), $2.31-2.08(\mathrm{~m}, 4 \mathrm{H}) .{ }^{13} \mathrm{C}\left\{{ }^{1} \mathrm{H}\right\}$ NMR (126 MHz, DMSO-d 6 ) $\delta 162.2(\mathrm{~d}, J=246 \mathrm{~Hz}), 133.2(\mathrm{~d}, J=8 \mathrm{~Hz}), 126.7$ (d, $J=3 \mathrm{~Hz}), 116.9$ (d, $J=22 \mathrm{~Hz}$ ), 44.7, 43.0, 28.6. HRMS (ESI-TOF) m/z: [M-BF $4^{-}$] calcd for $\left(\mathrm{C}_{11} \mathrm{H}_{14} \mathrm{FS}^{+}\right.$) 197.0800; Found 197.0804.

$7 \mathbf{h}$

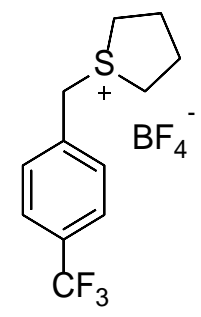

White Solid (1.51g, 45\%). Melting point: 95-98 ${ }^{\circ} \mathrm{C}$. IR: 3076, 2963, 1421, 1326, 1046, $1026 \mathrm{~cm}^{-1} .{ }^{1} \mathrm{H} \mathrm{NMR}(500 \mathrm{MHz}$, DMSO-d $\left.{ }_{6}\right) \delta 7.85$ (d, $\left.J=8.2 \mathrm{~Hz}, 2 \mathrm{H}\right), 7.80$ (d, $\left.J=8.2 \mathrm{~Hz}, 2 \mathrm{H}\right), 4.61$ (s, 2H), 3.49 (m, 2H), 3.40 (m, $\left.2 \mathrm{H}\right), 2.31-2.13$ (m, 4H). ${ }^{13} \mathrm{C}\left\{{ }^{1} \mathrm{H}\right\} \operatorname{NMR}\left(126 \mathrm{MHz}, \mathrm{DMSO}-d_{6}\right) \delta 135.3,131.8,130.4$ (q, $\left.J=32.0 \mathrm{~Hz}\right), 126.7$ (q, $\left.J=3.7 \mathrm{~Hz}\right), 125.5$, 44.7, 43.5, 28.6. HRMS (ESI-TOF) m/z: [M-BF $\left.4^{-}\right]$calcd for $\left(\mathrm{C}_{12} \mathrm{H}_{14} \mathrm{~F}_{3} \mathrm{~S}^{+}\right)$247.0763; Found 247.0769.

Synthesis of 1-(4-cyanobenzyl) tetrahydrothiophenium bromide salts (7i $)^{10}$

To a solution of dry dichloromethane $(30 \mathrm{~mL})$ in a $100 \mathrm{~mL}$ dry round bottom flask was added the 4-cyanobenzyl bromide ( 1 equiv, 20mmol) followed by tetrahydrothiophene (2 equiv, $40 \mathrm{mmol}$ ) for 30 minutes at $0{ }^{\circ} \mathrm{C}$. The mixture was stirred for 24 hours at room temperature. Evaporation of solvent under reduced pressure gave a colorless, thick oil which was precipitated with acetone to obtain the desired salt as a solid.<smiles>N#Cc1ccc(C[SH]2CCCC2Br)cc1</smiles> 
White Solid (2.67g, 47\%). Melting point: $103-105{ }^{\circ} \mathrm{C} .{ }^{1} \mathrm{H}$ NMR $\left(500 \mathrm{MHz}, \mathrm{DMSO}-\mathrm{d}_{6}\right) \delta 7.96(\mathrm{~d}, J=8.3 \mathrm{~Hz}, 2 \mathrm{H})$, 7.83 (d, $J=8.3 \mathrm{~Hz}, 2 \mathrm{H}), 4.70$ (s, 2H), 3.48 (m, 4H), $2.32-2.13$ (m, 4H). ${ }^{13} \mathrm{C}\left\{{ }^{1} \mathrm{H}\right\}$ NMR (126 MHz, DMSO-d $\left.\mathrm{d}_{6}\right) 136.3$, 133.6, 131.9, 118.8, 112.6, 44.7, 43.6, 28.7. HRMS (ESI-TOF) m/z: [M-Br-] calcd for $\left(\mathrm{C}_{12} \mathrm{H}_{14} \mathrm{NS}^{+}\right)$204.0841; Found 204.0848 .

Synthesis of N-(4-methoxyphenyl) benzimidamide (8a) ${ }^{11}$<smiles>COc1ccc(/N=C(/N)c2ccccc2)cc1</smiles>

Under air, a pressure flask (75 mL in volume) equipped with a stirring bar was charged with $p$-methoxyphenylamine (1.0 equiv, $20 \mathrm{mmol}$ ) followed by benzonitrile (1.0 equiv, $20 \mathrm{mmol}$ ). $\mathrm{AlCl}_{3}$ (1.0 equiv, $20 \mathrm{mmol}$ ) was added in one portion while stirring the reaction. The flask was tightly sealed with a screw cap and placed into a preheated oil bath at $150{ }^{\circ} \mathrm{C}$ (Safety shield is recommended while running this reaction). The reaction mixture was stirred for 50 min, and subsequently taken out of the oil bath. $20 \mathrm{~mL}$ of $1 \mathrm{M}$ ice cold hydrochloric acid ( $\mathrm{HCl}$ ) solution was then added after 2 minutes to the mixture under vigorous stirring. Then, concentrated aqueous $\mathrm{NaOH}$ was added until a pH reached above 12. The aqueous layer was extracted with dichloromethane ( $3 \mathrm{X} 50 \mathrm{~mL}$ ). The combined organic layers were washed with water and then brine, and dried over $\mathrm{Na}_{2} \mathrm{SO}_{4}$, filtered, and concentrated to $20 \mathrm{~mL}$ under reduced pressure. After that, $20 \mathrm{~mL}$ of hexane was added in the crude organic layer to precipitate out a solid product. The precipitate was washed with hexane and the desired product was collected and confirmed by NMR.

Whitish pink solid (2.94g, 65\%), Melting point:115-116 ${ }^{\circ} \mathrm{C}$. IR: 3330, 3281, 3046, 2978, 1653, 1610, 1516, 1269, $1089 \mathrm{~cm}^{-1} .{ }^{1} \mathrm{H}$ NMR (500 MHz, DMSO-d $\left.d_{6}\right) \delta 7.95$ (d, $\left.J=7.5 \mathrm{~Hz}, 2 \mathrm{H}\right), 7.48-7.36$ (m, 3H), 6.89 (d, $\left.J=8.7 \mathrm{~Hz}, 2 \mathrm{H}\right)$, 6.78 (d, $J=7.5 \mathrm{~Hz}, 2 \mathrm{H}), 6.19$ (s, 2H), 3.72 (s, 3H). ${ }^{13} \mathrm{C}\left\{{ }^{1} \mathrm{H}\right\}$ NMR (126 MHz, DMSO-d 6 ) $\delta$ 154.9, 154.4, 144.0, 136.5, 130.4, 128.4, 127.4, 122.8, 115.0, 55.5. HRMS (ESI-TOF) m/z: $\left[(\mathrm{M}+\mathrm{H})^{+}\right]$calcd for $\left(\mathrm{C}_{14} \mathrm{H}_{15} \mathrm{~N}_{2} \mathrm{O}^{+}\right)$227.1179; Found 227.1180.

Synthesis of 1,3-diaza-1,3-butadiene $(\mathbf{8 b})^{12}$<smiles>COc1ccc(/N=C(\N=C\c2ccccc2)c2ccccc2)cc1</smiles>

To a solution of dry toluene $(30 \mathrm{~mL})$ in a $100 \mathrm{~mL}$ dry round bottom flask was added $N$-(4-methoxyphenyl) benzimidamide (8a), followed by benzaldehyde (1.1 equiv) and triethylamine (2.75 equiv). The solution was subsequently cooled to $0{ }^{\circ} \mathrm{C}$ under a nitrogen atmosphere. Then $\mathrm{TiCl}_{4}(0.55$ equiv) in dry toluene (1M solution in toluene) was slowly added dropwise for 90 min under a nitrogen atmosphere. After that, the mixture was stirred for 
additional 12 hours. The resulting white yellow suspension was rapidly filtered using an aspirator pressure through a Celite pad and the clear liquid was stored in a sealed flask overnight at $4-6{ }^{\circ} \mathrm{C}$. The cold cloudy solution was re-filtered under reduced pressure over a well-pressed Celite pad and freed from solvent under reduced pressure without heating to give desire $N$-(benzylidene)- $N$ '-(4-methoxyphenyl) benzimidamide as a mixture compound, which is used in next step without further purification.

${ }^{1} \mathrm{H}$ NMR (500 MHz, $\left.\mathrm{CHCl}_{3}-d\right) \delta 8.19(\mathrm{~s}, 1 \mathrm{H}), 7.97-7.94(\mathrm{~m}, 2 \mathrm{H}), 7.79-7.76(\mathrm{~m}, 2 \mathrm{H}), 7.26-7.24(\mathrm{~m}, 2 \mathrm{H}), 7.19-$ 7.17 (m, 4H), $7.03-6.99$ (d, $J=9 \mathrm{~Hz}, 2 \mathrm{H}), 6.79-6.77$ (d, $J=9 \mathrm{~Hz}, 2 \mathrm{H}), 3.74$ (s, 3H). ${ }^{13} \mathrm{C}\left\{{ }^{1} \mathrm{H}\right\}$ NMR $(126 \mathrm{MHz}$, $\left.\mathrm{CHCl}_{3}-d\right) \delta 162.7,162.2,158.4,137.9,132.3,130.7,129.0,128.9,128.7,128.3,128.1,125.3,124.2,113.8,55.3$. HRMS (ESI-TOF) m/z: [(M+H) $]$ calcd for $\left(\mathrm{C}_{21} \mathrm{H}_{19} \mathrm{~N}_{2} \mathrm{O}^{+}\right)$315.1492; Found 315.1499.

\section{REFERENCES}

(1) Aggarwal, V. K.; Vasse, J.-L. Asymmetric Sulfur Ylide Mediated Aziridination: Application in the Synthesis of the Side Chain of Taxol. Org. Lett. 2003, 5 (21), 3987-3990. https://doi.org/10.1021/ol035554w.

(2) Katritzky, A. R.; Fan, W. Q.; Black, M.; Pernak, J. N-[1-(Benzotriazol-1-Yl)alkyl]amides, Versatile Amidoalkylation Reagents. 5. A General and Convenient Route to N-(.alpha.-Alkoxyalkyl)amides. J. Org. Chem. 1992, 57 (2), 547-549. https://doi.org/10.1021/jo00028a028.

(3) Katritzky, A. R.; Pernak, J.; Fan, W. Q.; Saczewski, F. N-(1-Benzotriazol-1-Ylalkyl)amides, Versatile .alpha.Amidoalkylation Reagents. 1. .alpha.-Amidoalkylation of CH Acids. J. Org. Chem. 1991, 56 (14), 4439-4443. https://doi.org/10.1021/jo00014a020.

(4) Forrester, J.; Jones, R. V. H.; Newton, L.; Preston, P. N. Synthesis and Reactivity of Benzylic Sulfonium Salts: Benzylation of Phenol and Thiophenol under near-Neutral Conditions. Tetrahedron 2001, 57 (14), 2871-2884. https://doi.org/10.1016/S0040-4020(01)00137-5.

(5) Murry, J. A.; Frantz, D. E.; Soheili, A.; Tillyer, R.; Grabowski, E. J. J.; Reider, P. J. Synthesis of $\alpha$-Amido Ketones via Organic Catalysis: Thiazolium-Catalyzed Cross-Coupling of Aldehydes with Acylimines. J. Am. Chem. Soc. 2001, 123 (39), 9696-9697. https://doi.org/10.1021/ja0165943.

(6) Padwa, A.; Gasdaska, J. R. Generation of Sulfur Ylides by the Desilylation of $\alpha$-Trimethylsilylbenzyl Sulfonium Salts. Tetrahedron 1988, 44 (13), 4147-4156. https://doi.org/10.1016/S0040-4020(01)86662-X.

(7) Aggarwal, V. K.; Fang, G. Y.; Schmidt, A. T. Synthesis and Applications of Chiral Organoboranes Generated from Sulfonium Ylides. J. Am. Chem. Soc. 2005, 127 (6), 1642-1643. https://doi.org/10.1021/ja043632k.

(8) Fang, G. Y.; Aggarwal, V. K. Asymmetric Synthesis of $\alpha$-Substituted Allyl Boranes and Their Application in the Synthesis of Iso-Agatharesinol. Angew. Chem. Int. Ed. 2007, 46 (3), 359-362. https://doi.org/10.1002/anie.200603659.

(9) Clergue, S.; Rousseau, O.; Delaunay, T.; Dequirez, G.; Tran, T.-V.; El Aakchioui, S.; Barozzino-Consiglio, G.; Robiette, R. Asymmetric Sulfur-Ylide-Mediated Formal [4+1]-Annulation Reaction: Scope and Mechanism. Chem. - Eur. J. 2018, 24 (44), 11417-11425. https://doi.org/10.1002/chem.201801874.

(10) Okazaki, Y.; Ando, F.; Koketsu, J. Generation of Sulfur Ylides from Sulfonium Salts and Their Reactions. Comparative Study of Electrochemical Reduction with the Base Method and Mechanism Elucidation by the MO Method. Bull. Chem. Soc. Jpn. 2003, 76 (11), 2155-2165. https://doi.org/10.1246/bcsj.76.2155.

(11) Veer, S. D.; Katkar, K. V.; Akamanchi, K. G. Sulfated Tungstate Catalyzed Activation of Nitriles: Addition of Amines to Nitriles for Synthesis of Amidines. Tetrahedron Lett. 2016, 57 (36), 4039-4043. https://doi.org/10.1016/j.tetlet.2016.07.073.

(12) Abbiati, G.; Contini, A.; Nava, D.; Rossi, E. Cycloaddition Reactions of 1,3-Diazabuta-1,3-Dienes with Alkynyl Ketenes. Tetrahedron 2009, 65 (24), 4664-4670. https://doi.org/10.1016/j.tet.2009.04.041. 


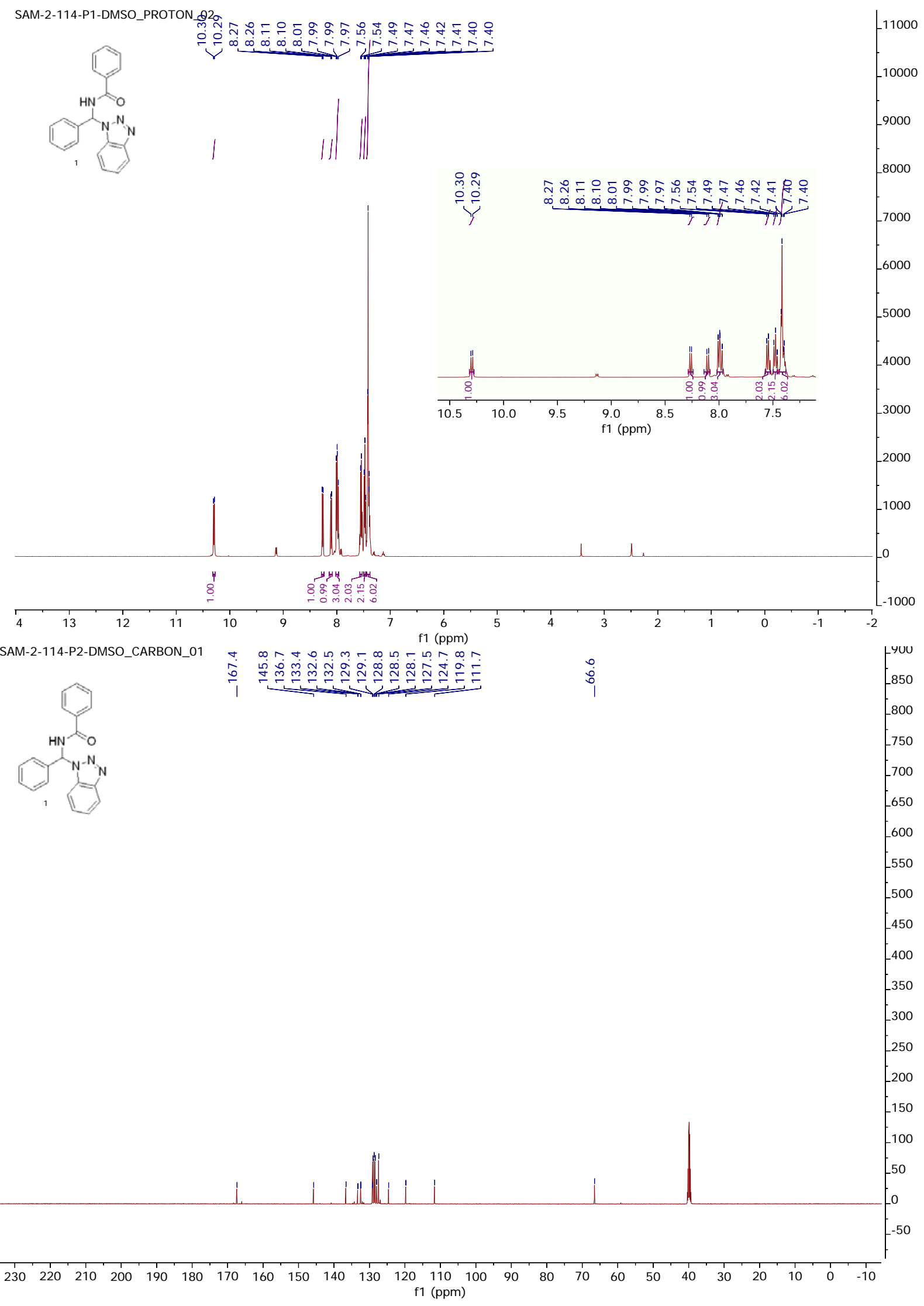

S15 


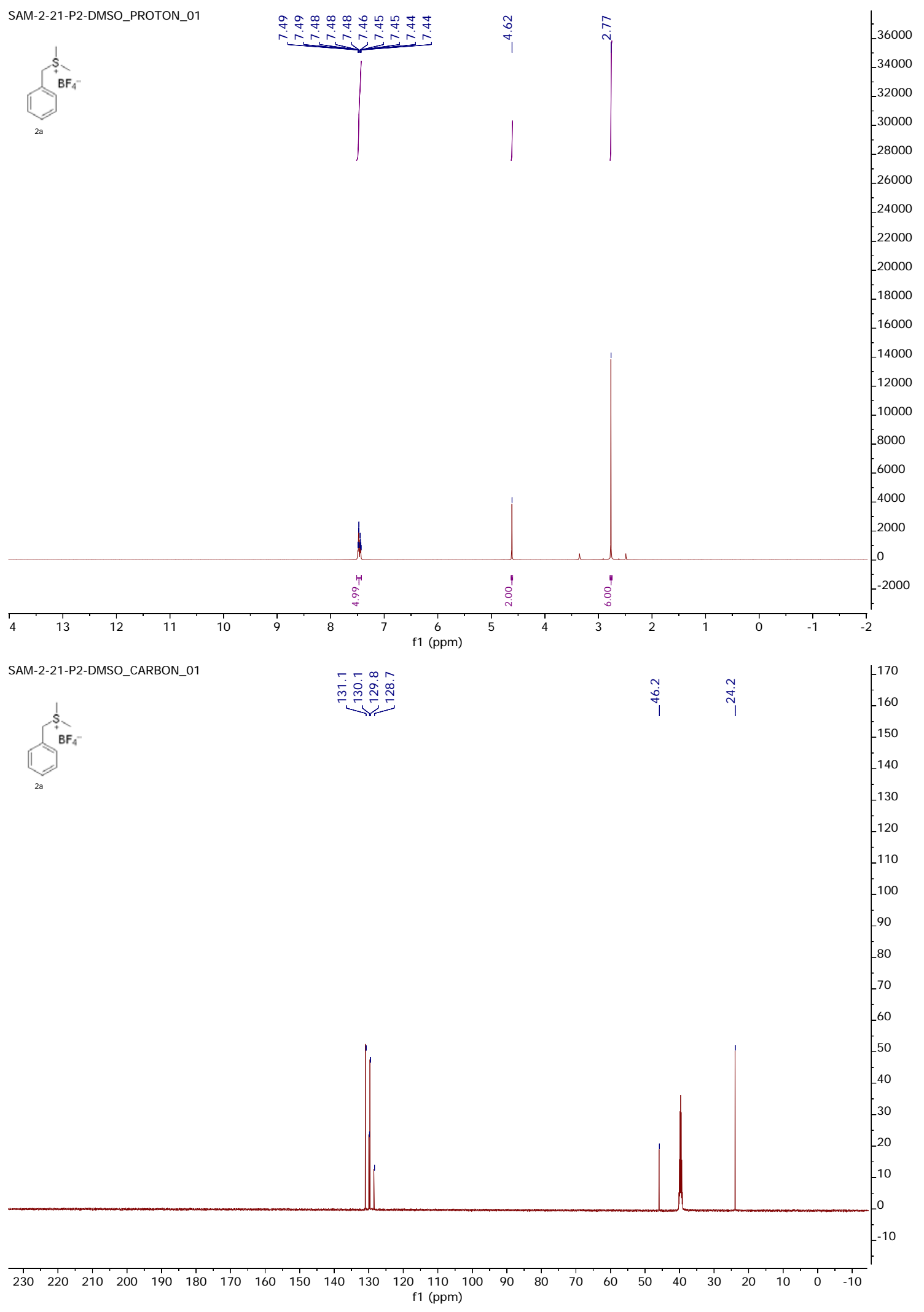




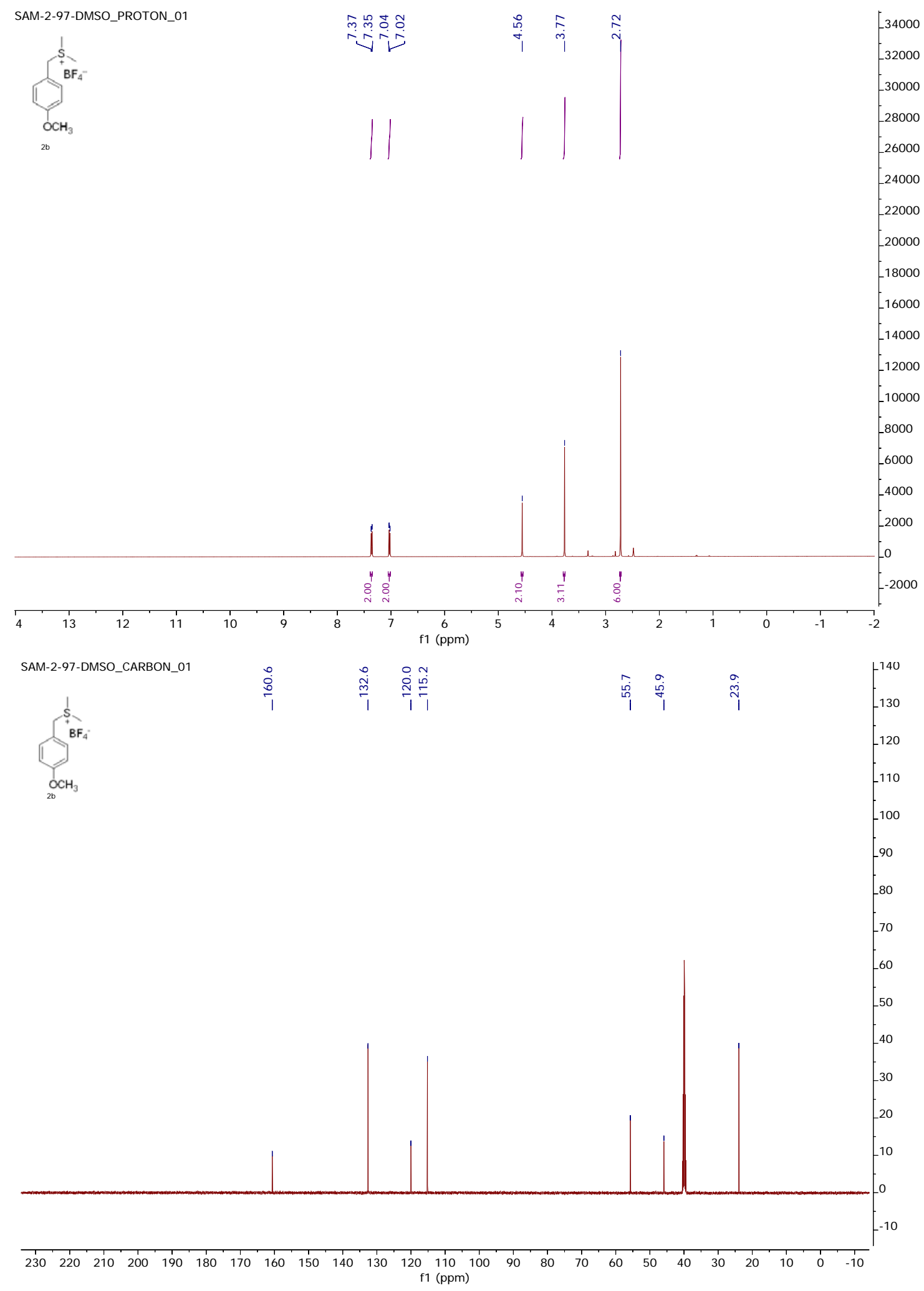




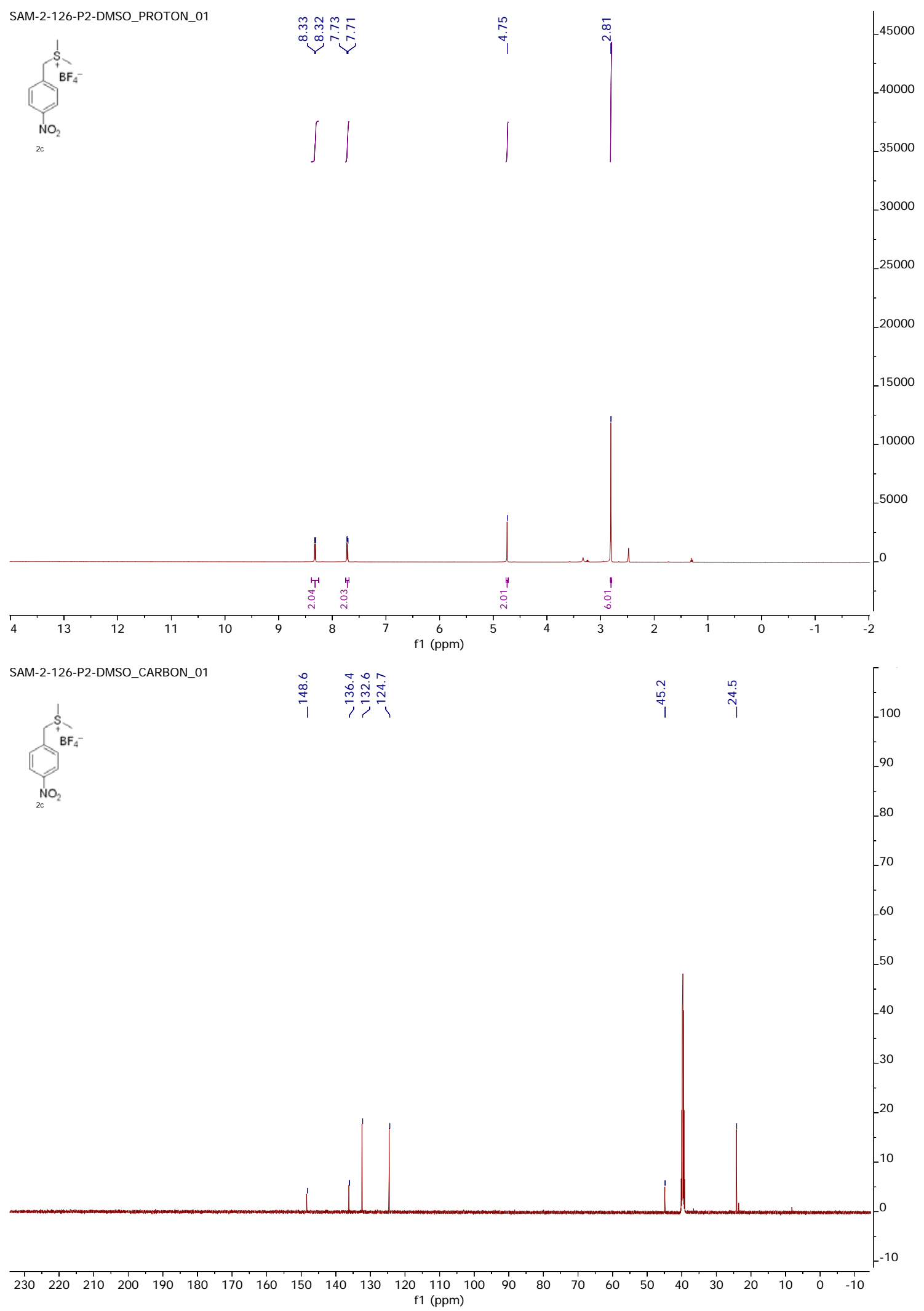

S18 


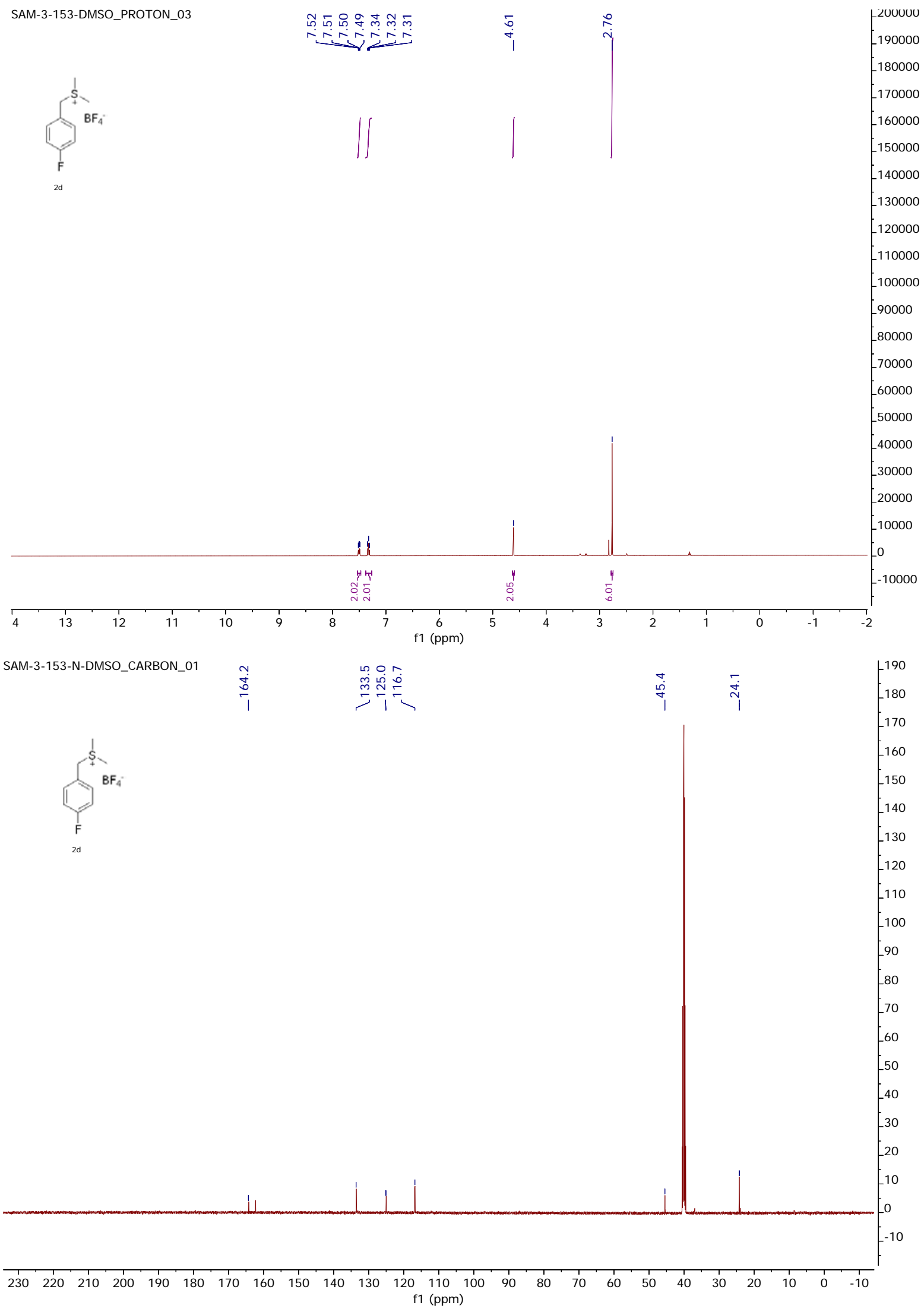



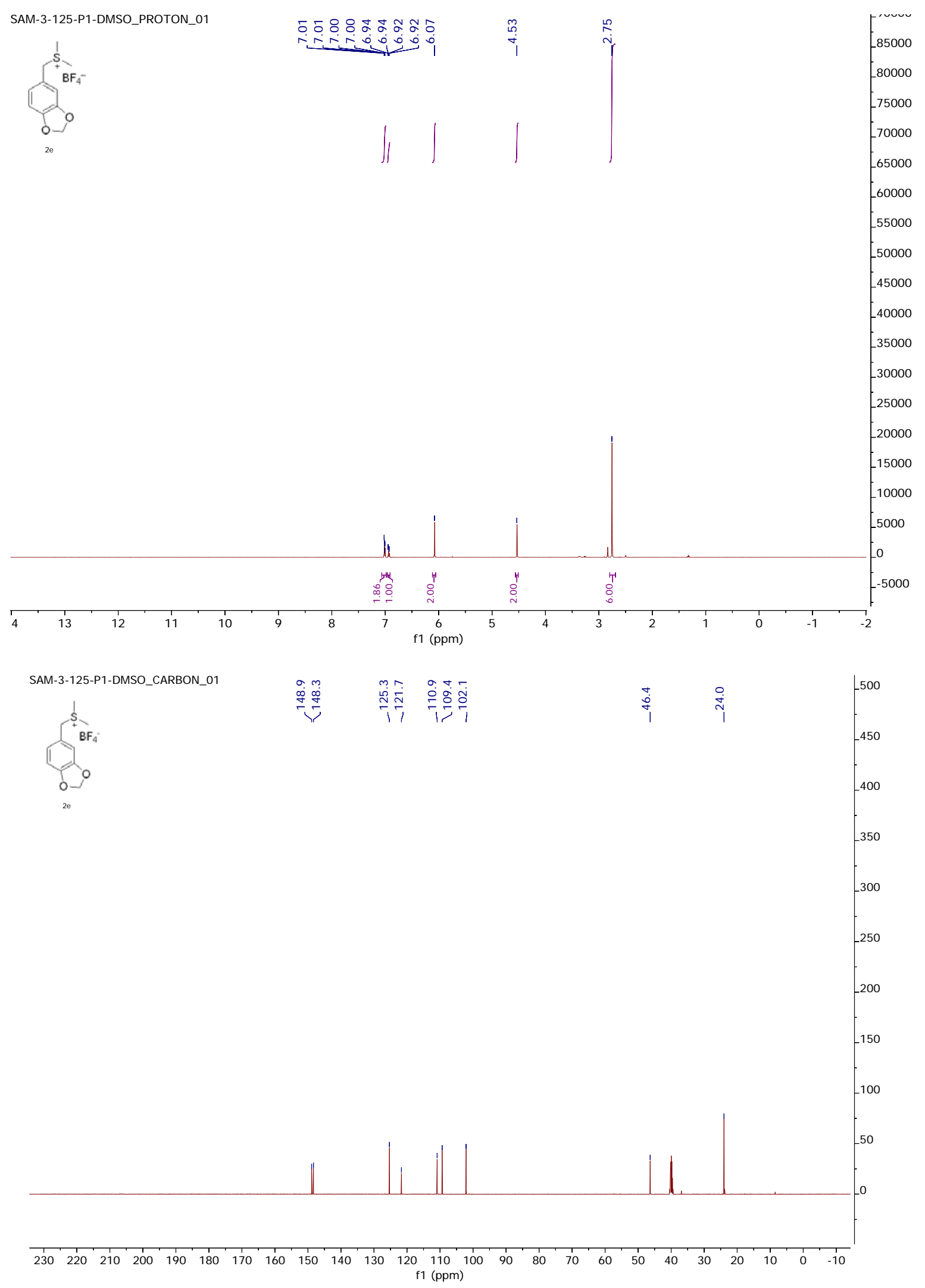


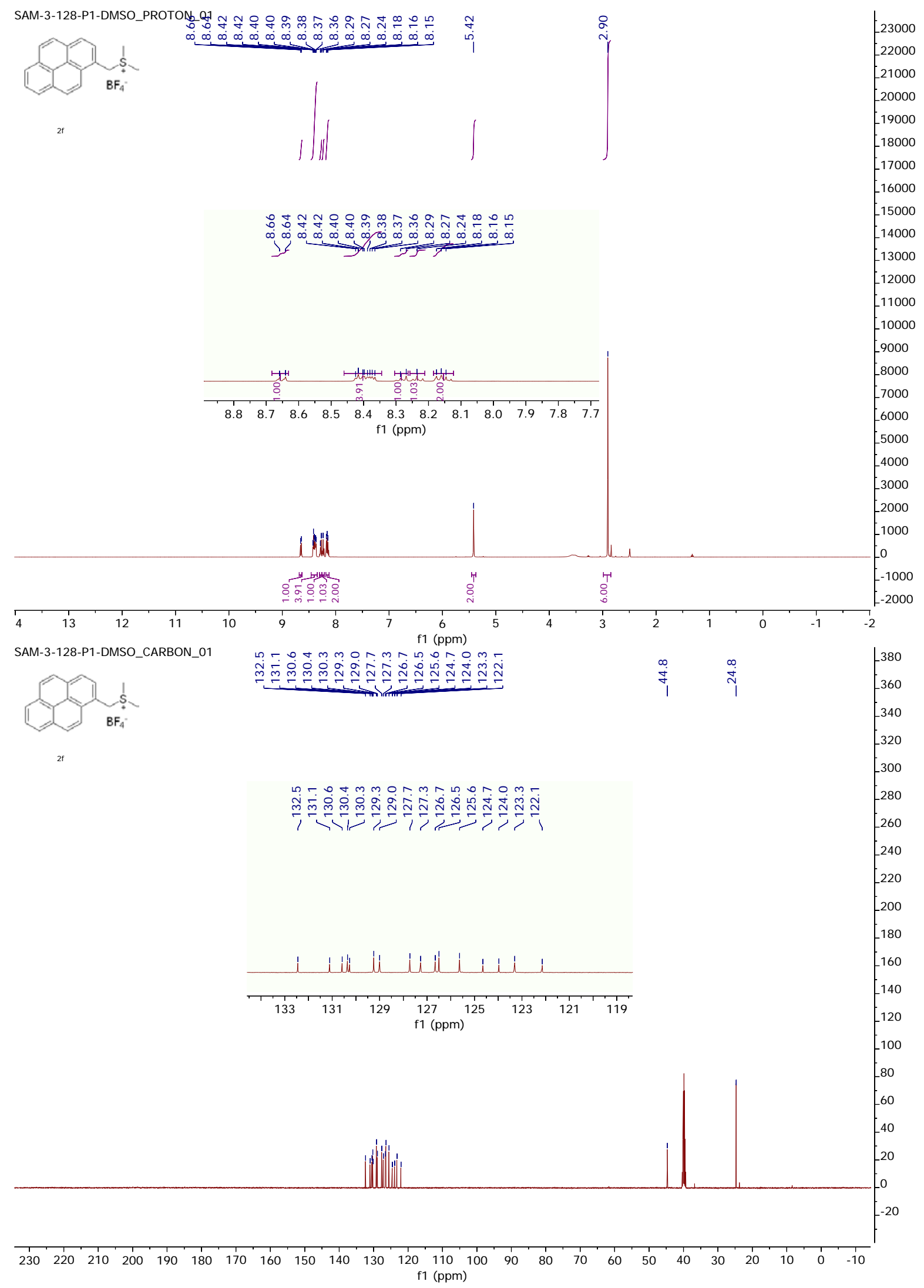




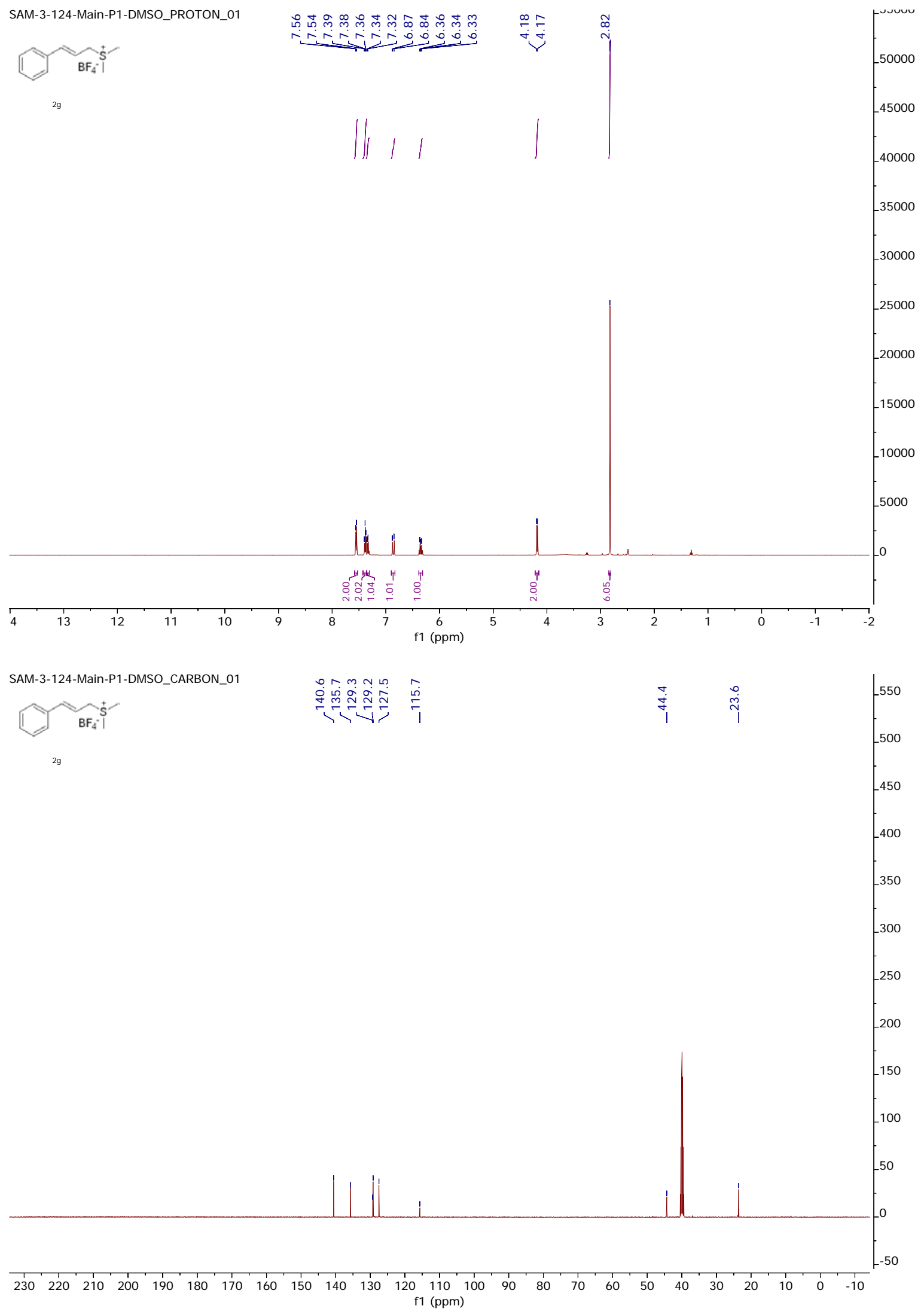




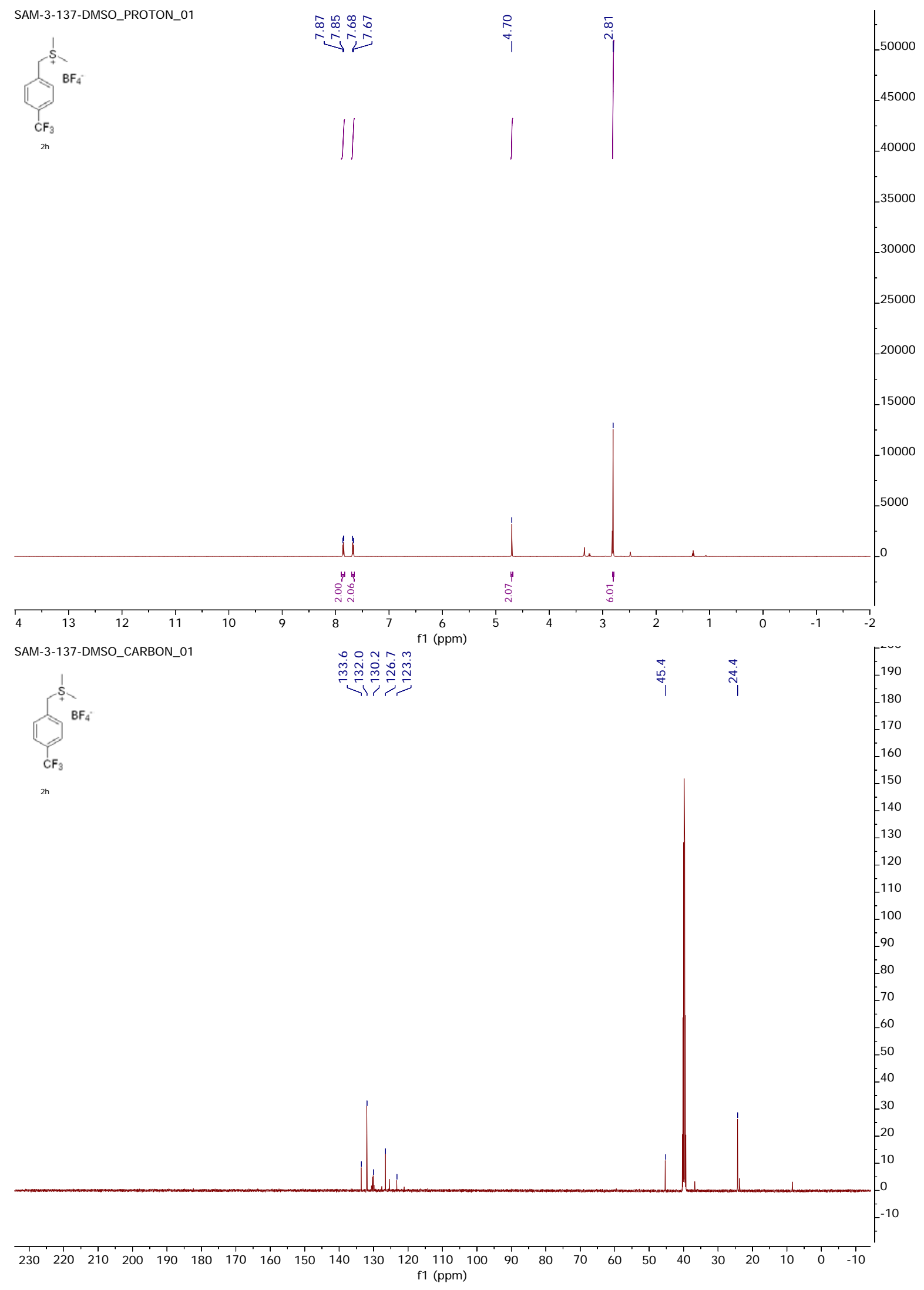




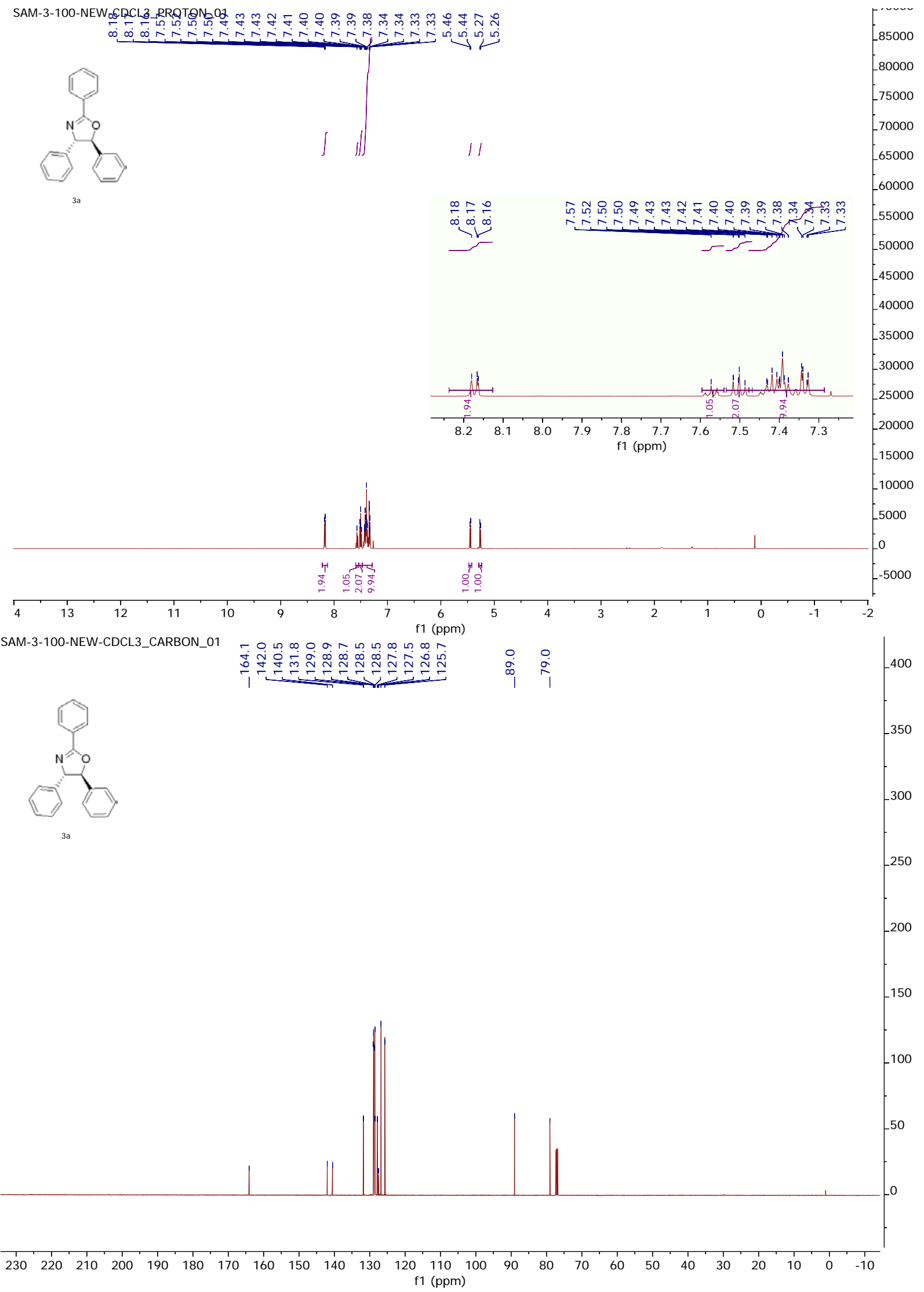




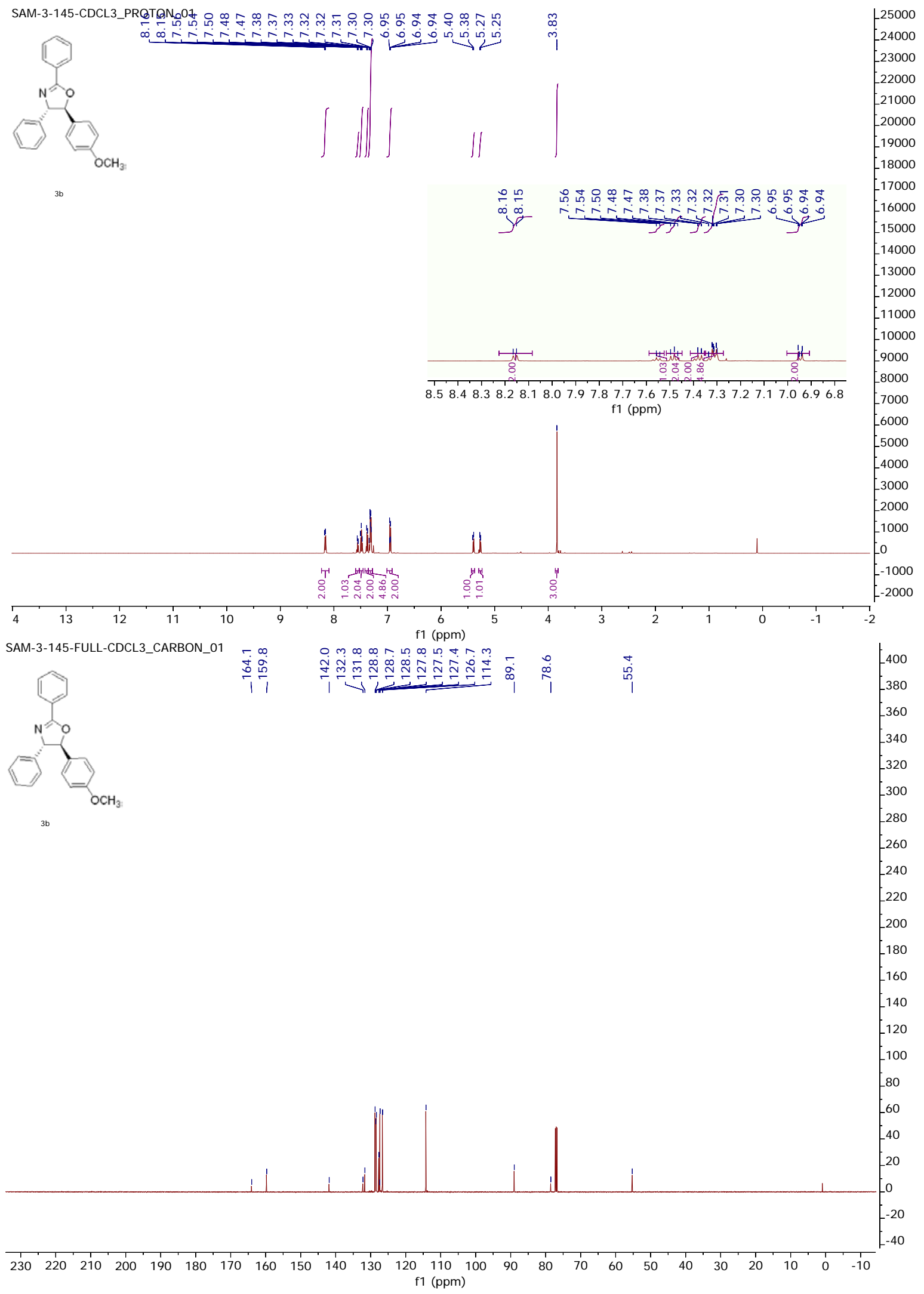




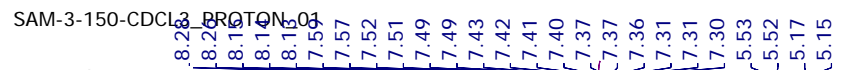
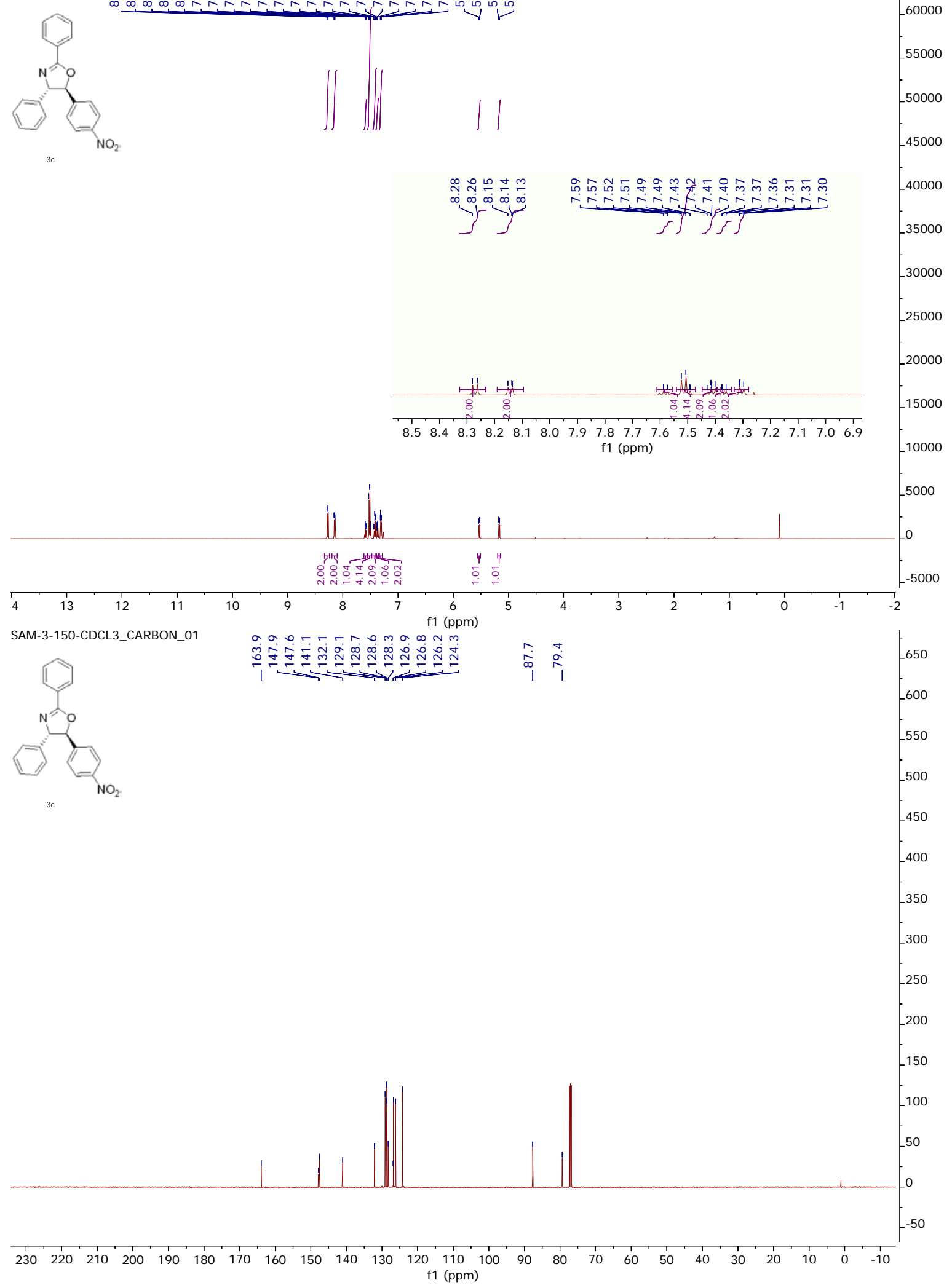


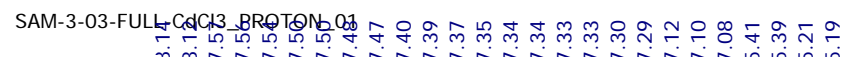

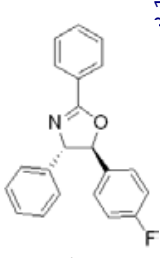

o

$3 d$
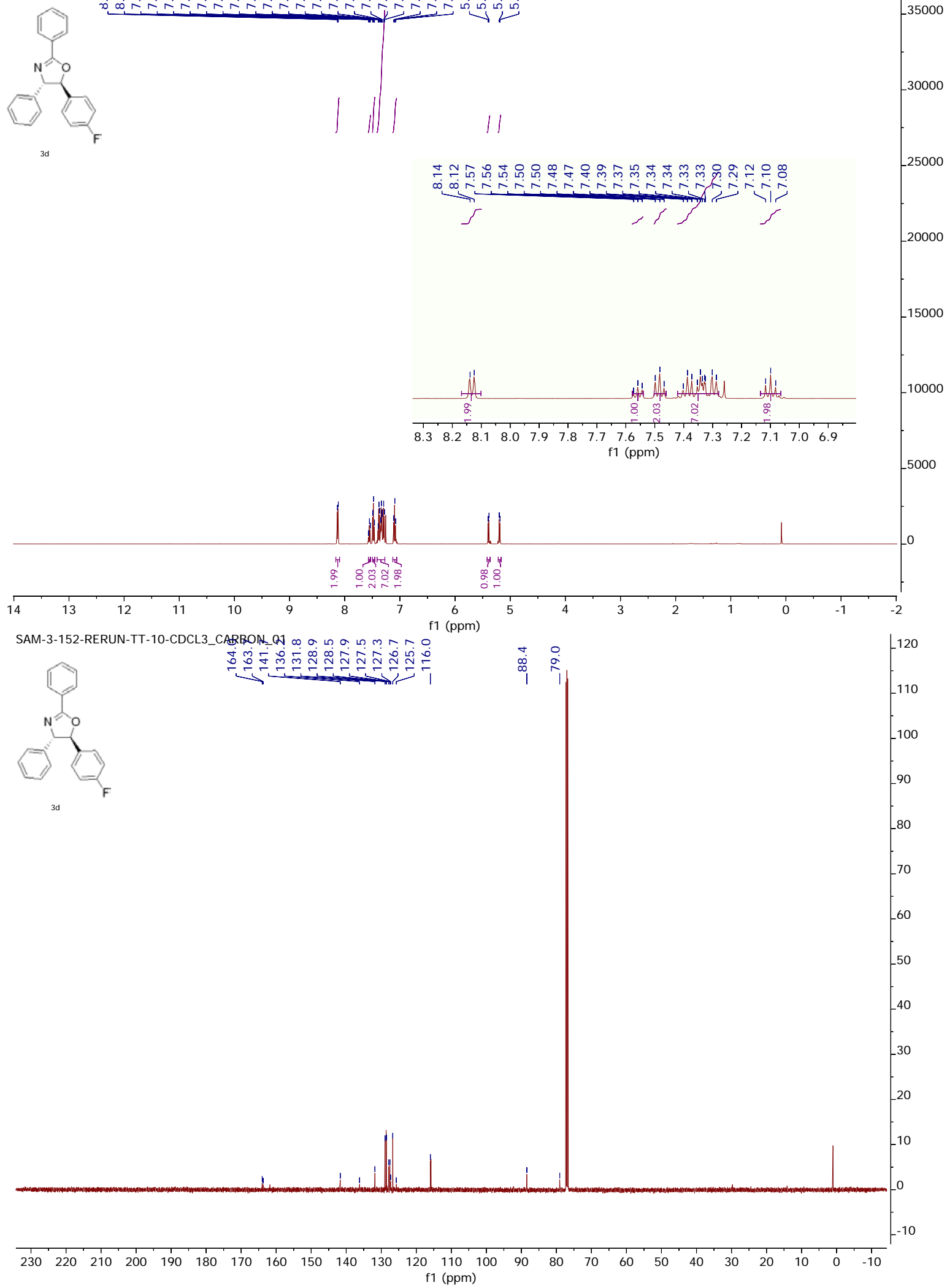
SAM-3-146-CDGL 3.

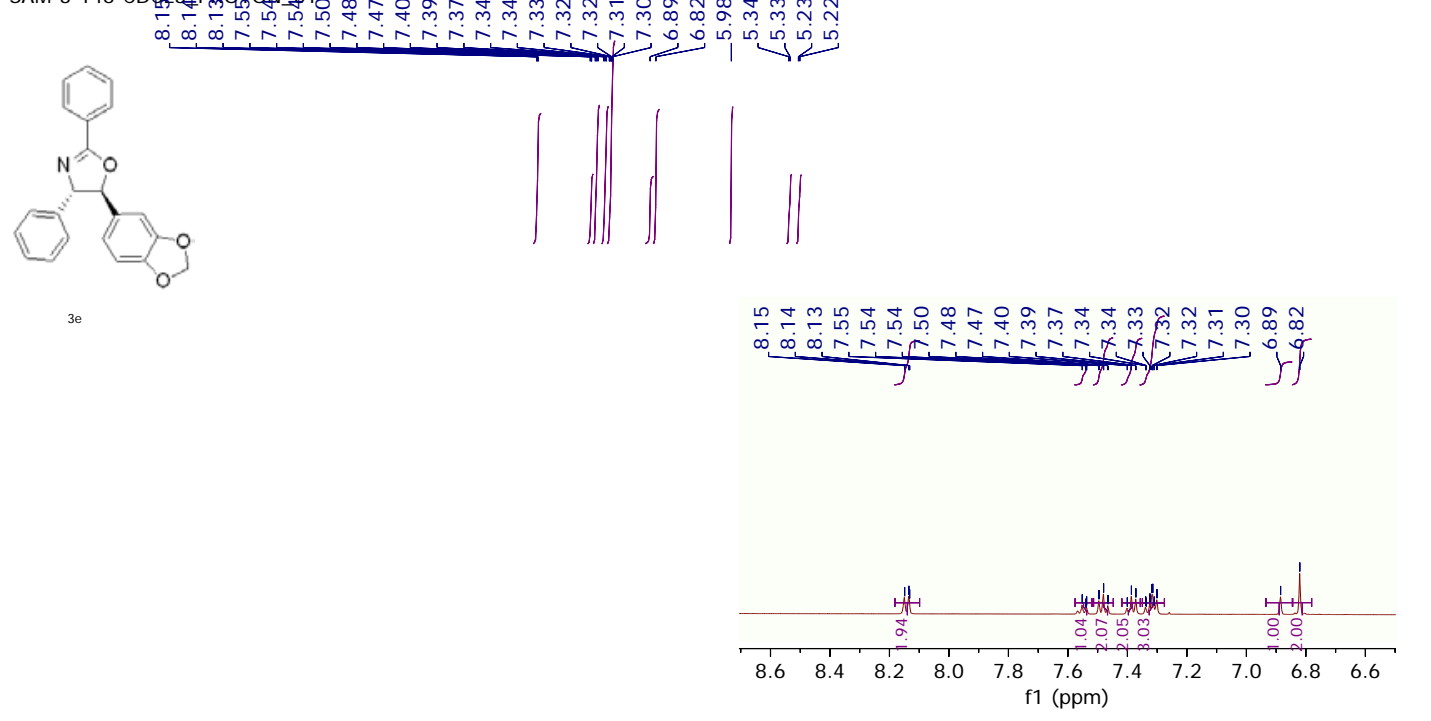

\begin{tabular}{lllllllllllllllll}
\hline & 13 & 12 & 11 & 10 & 9 & 8 & 7 & 6 & 5 & 4 & 3 & 2 & 1 & 0 & -1 & -2
\end{tabular}

\section{SAM-3-146-CDCL3_CARBON_01}
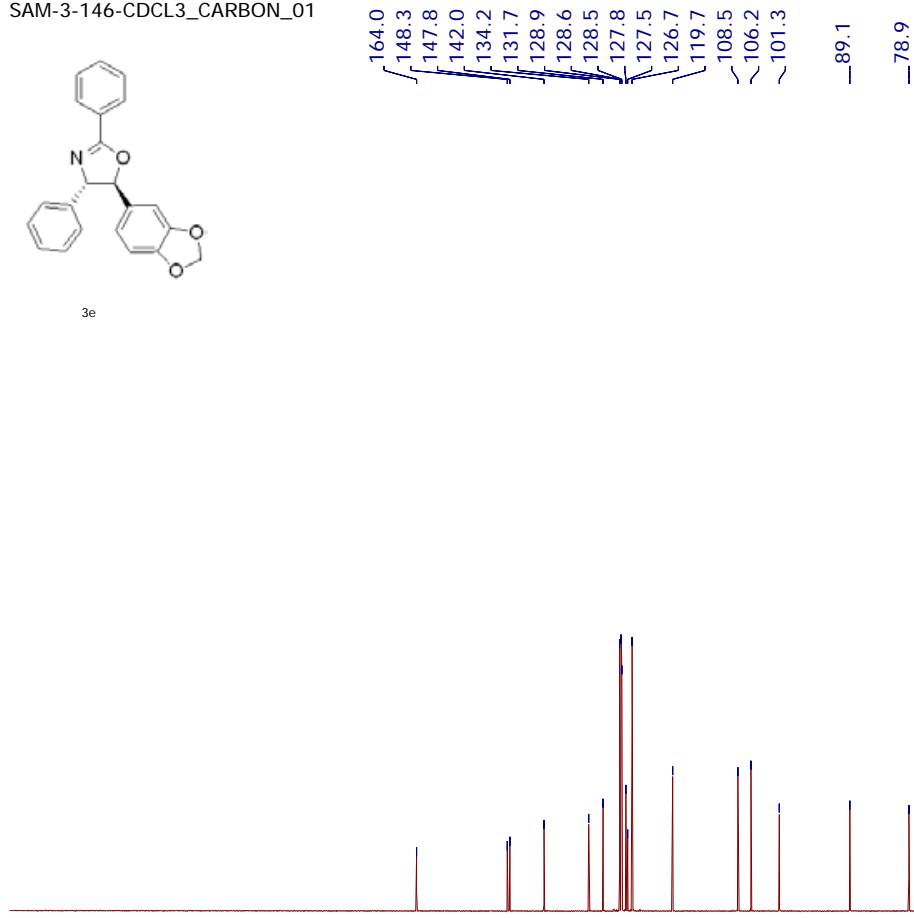


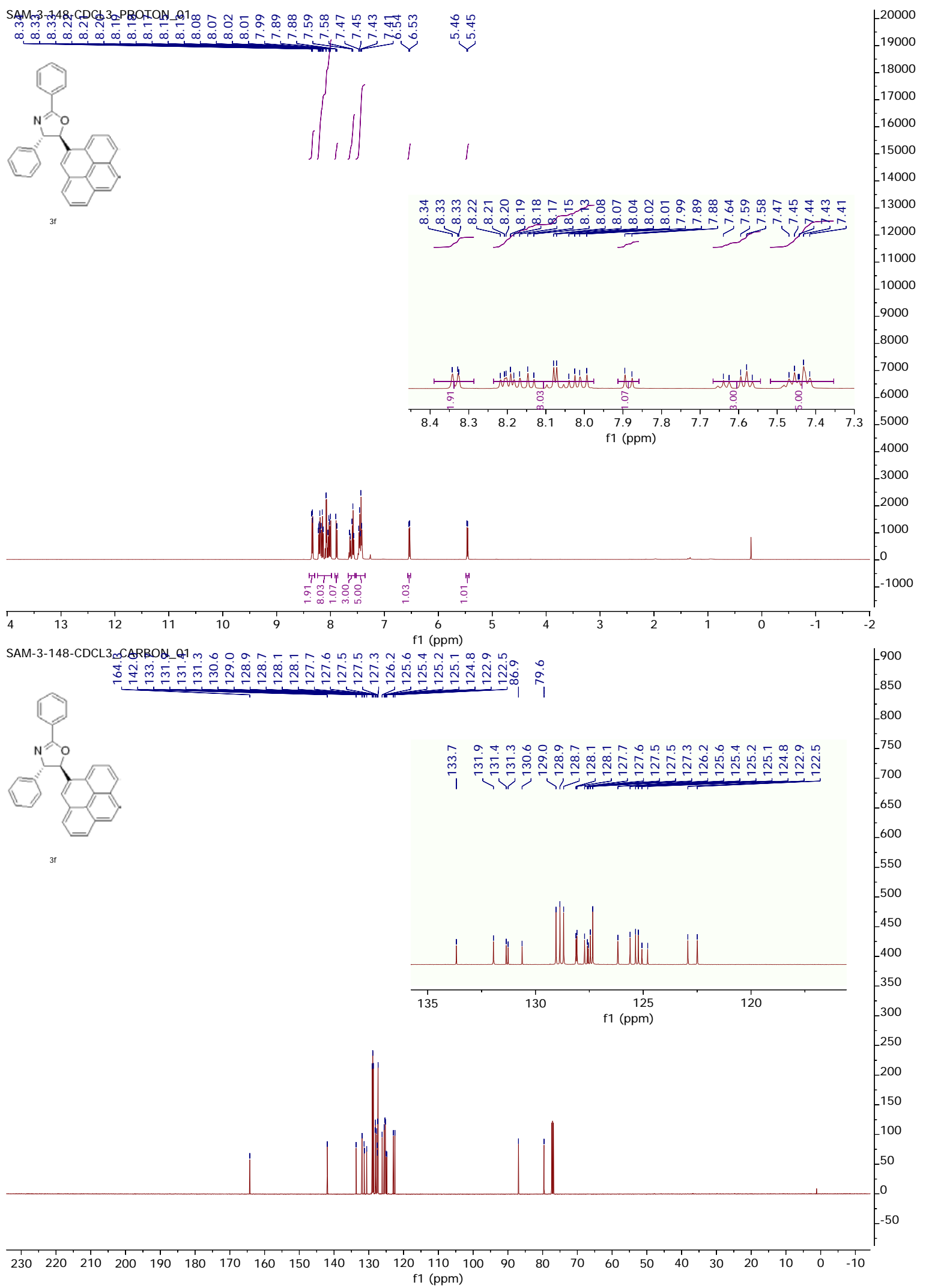


SAM-
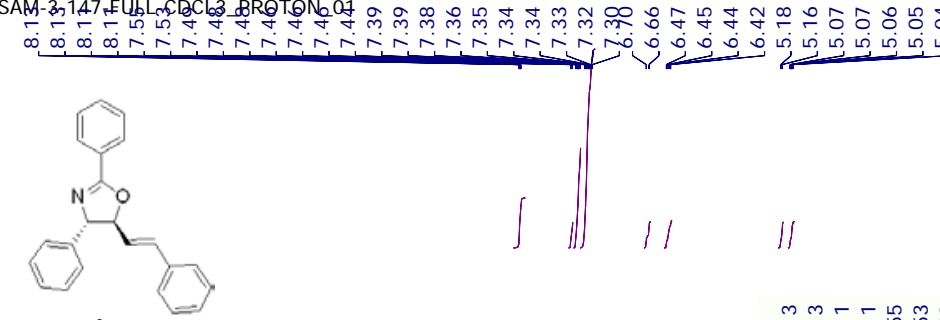

26000

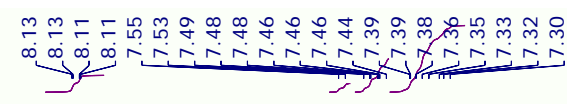

24000

22000

20000

18000

16000

14000

12000

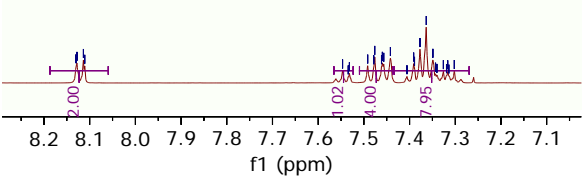

10000

8000

6000

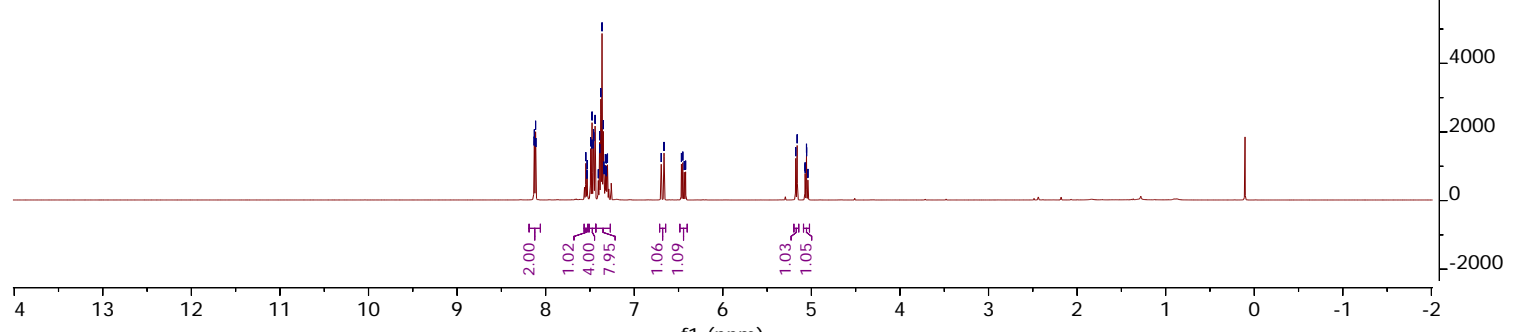

$\begin{array}{llllll}4 & 13 & 12 & 11 & 10 & 9\end{array}$

SAM-3-147-FULL-CDCL3_CARBON_01

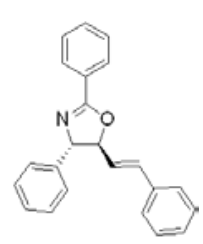

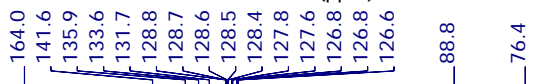

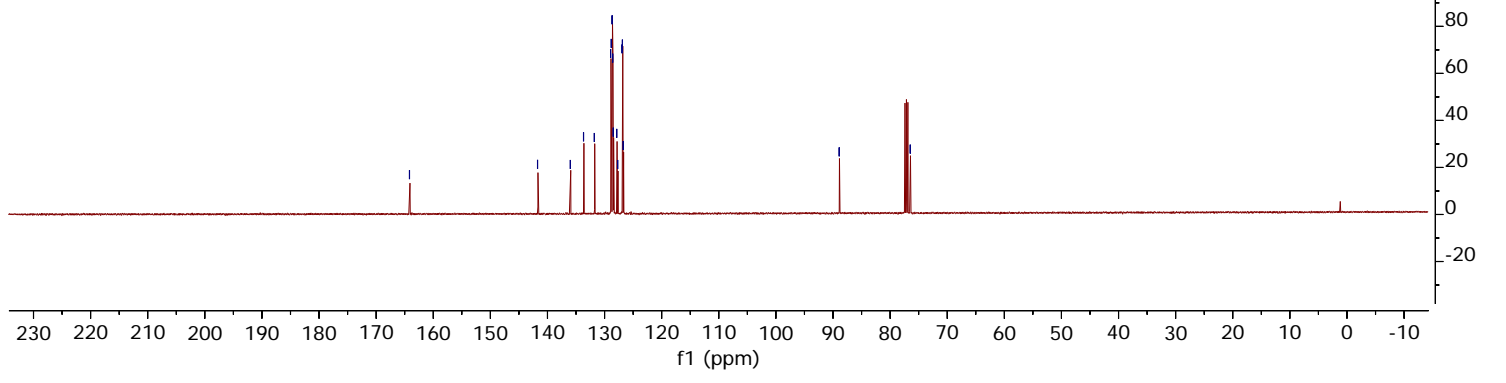




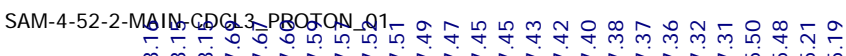
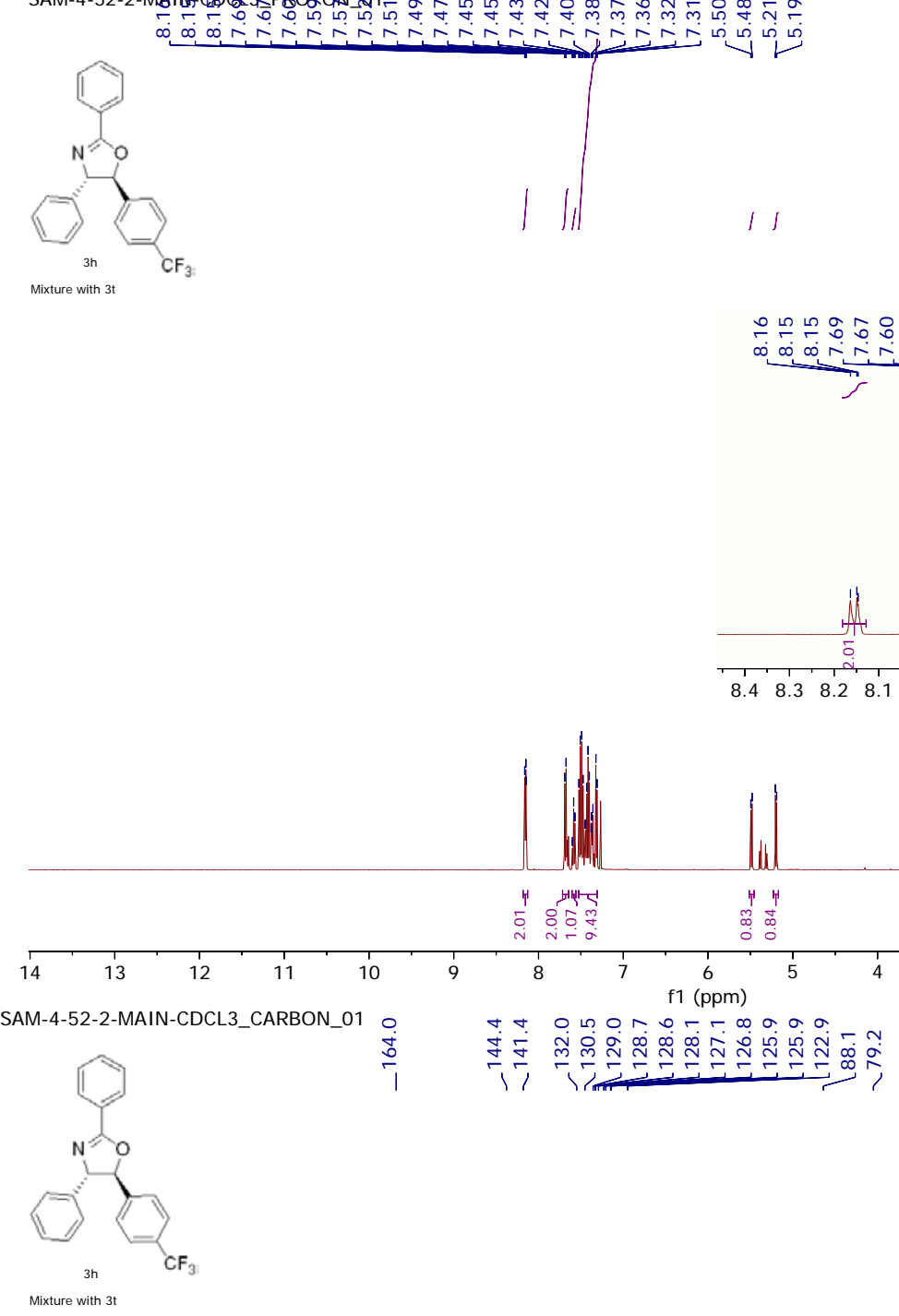

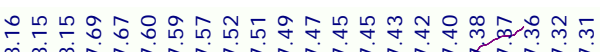

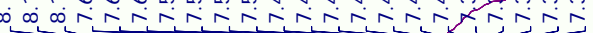
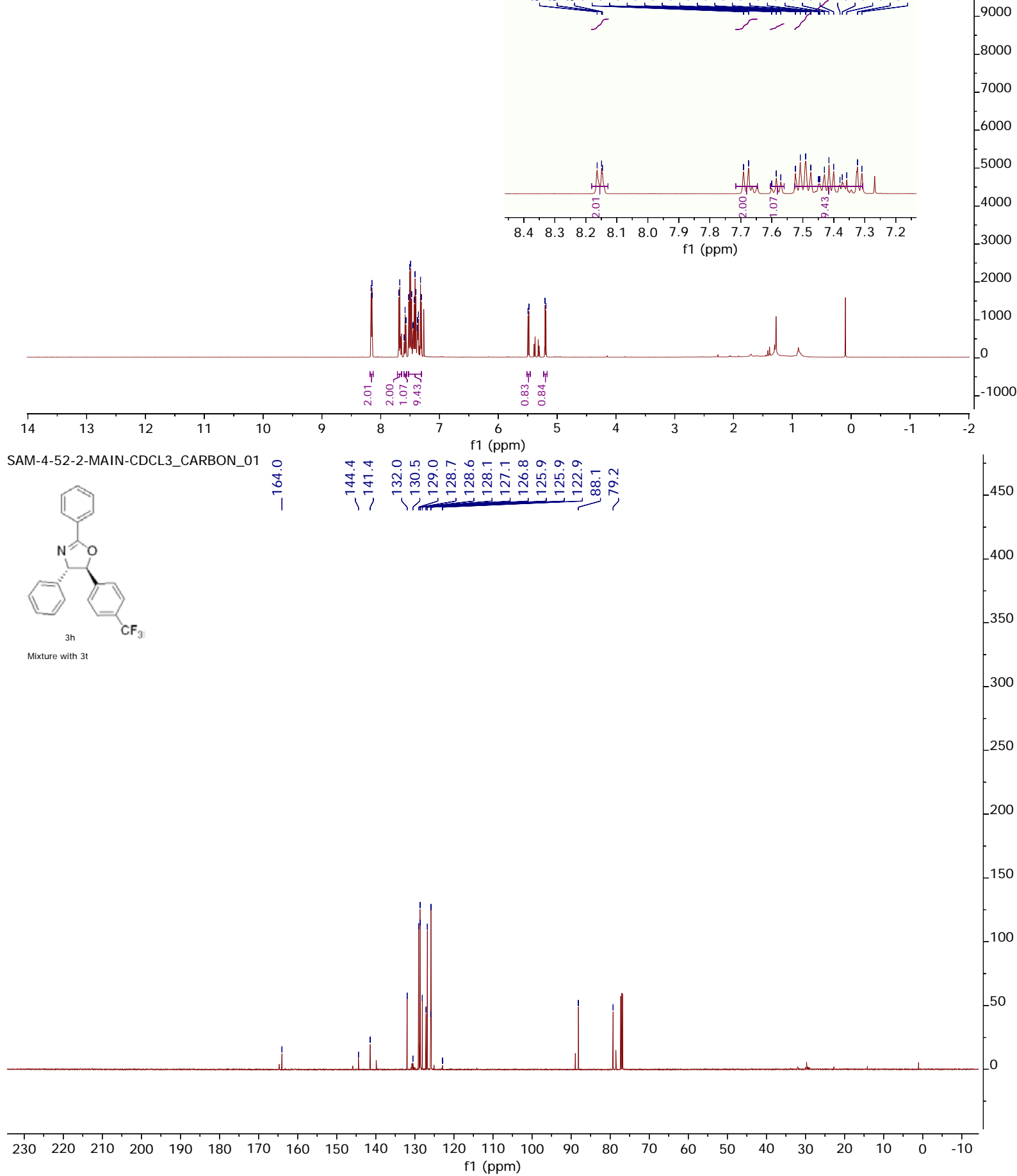


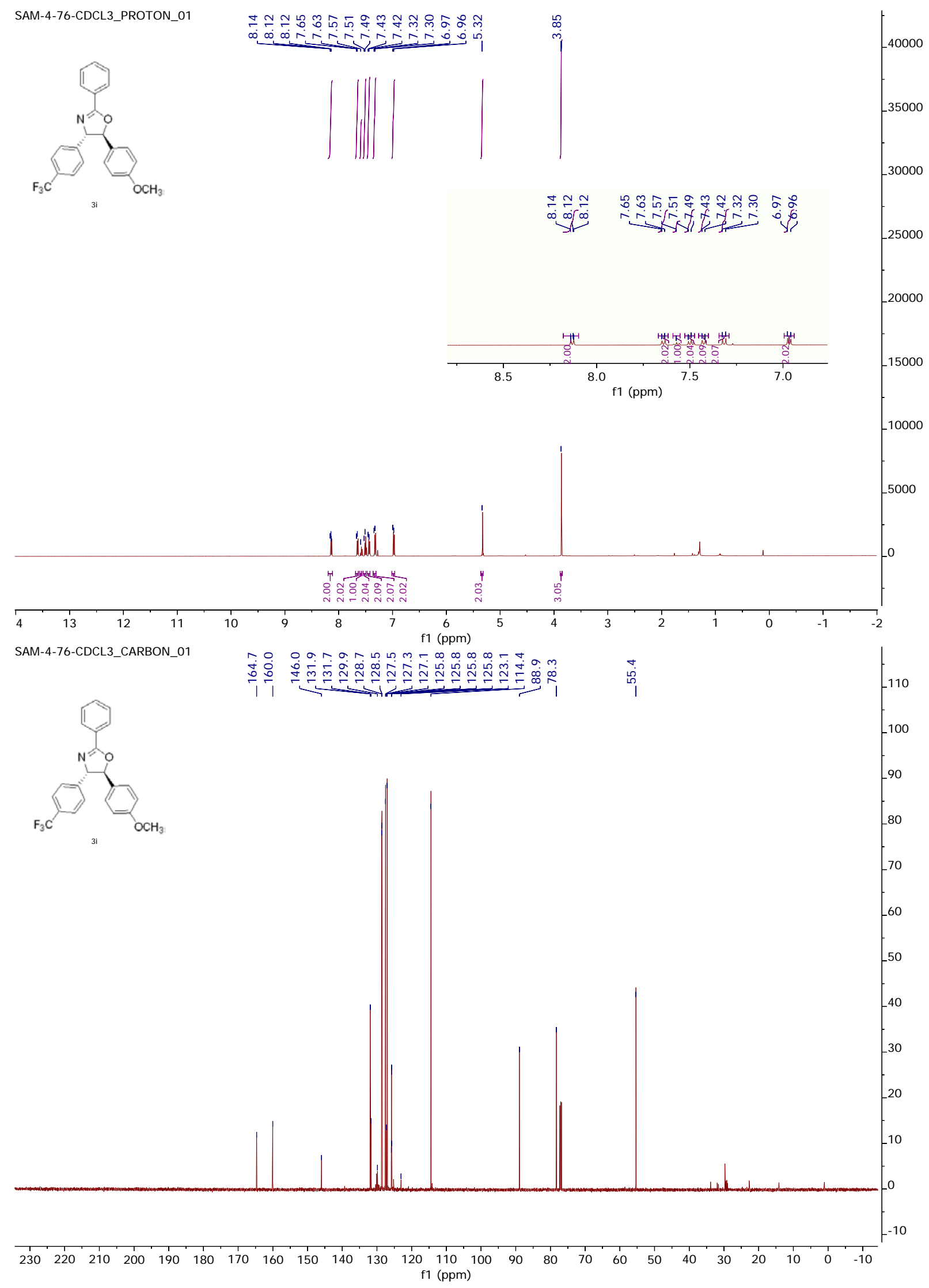




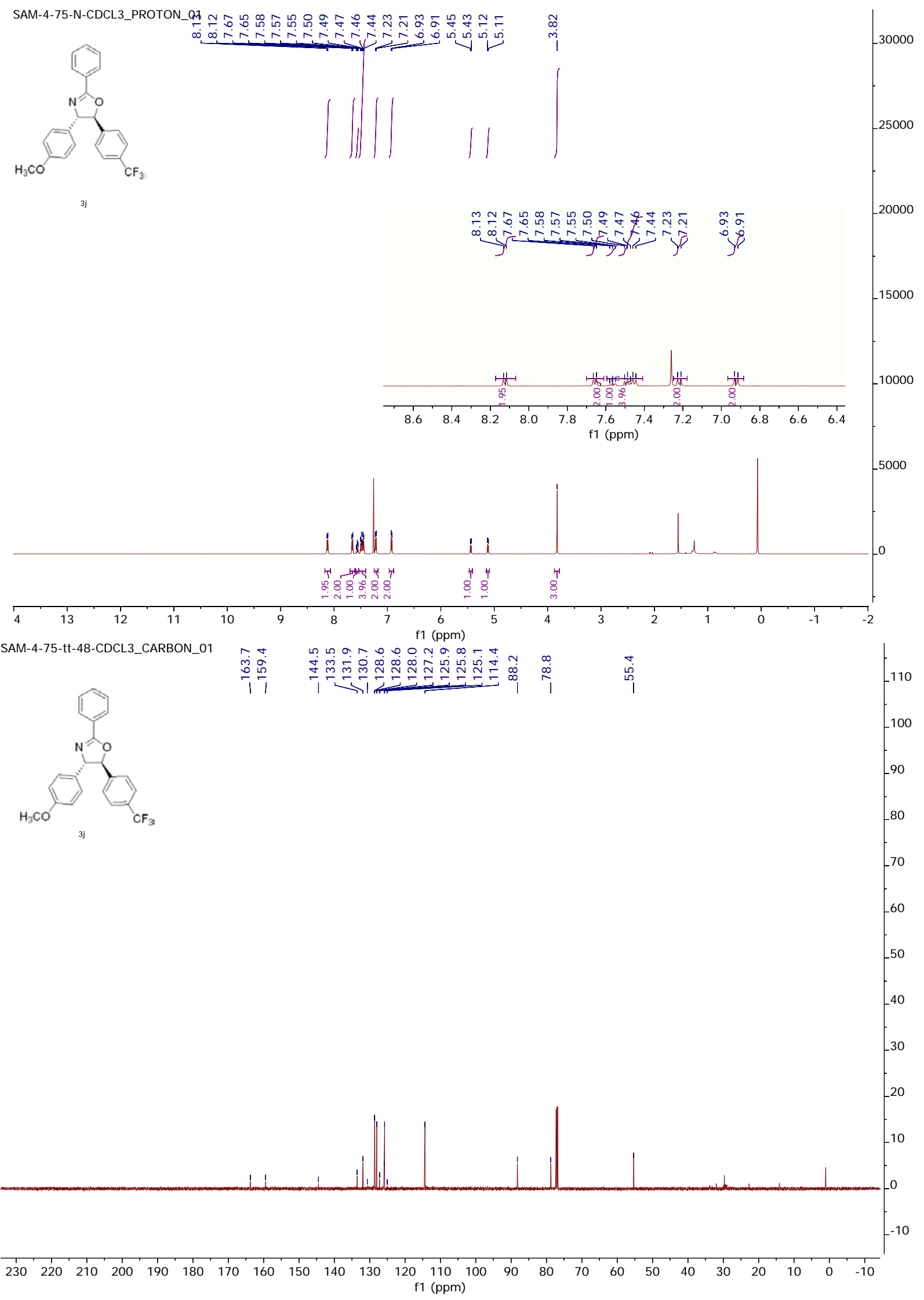


SAM-4-63-CDCL3_PROTQA_LA2m m
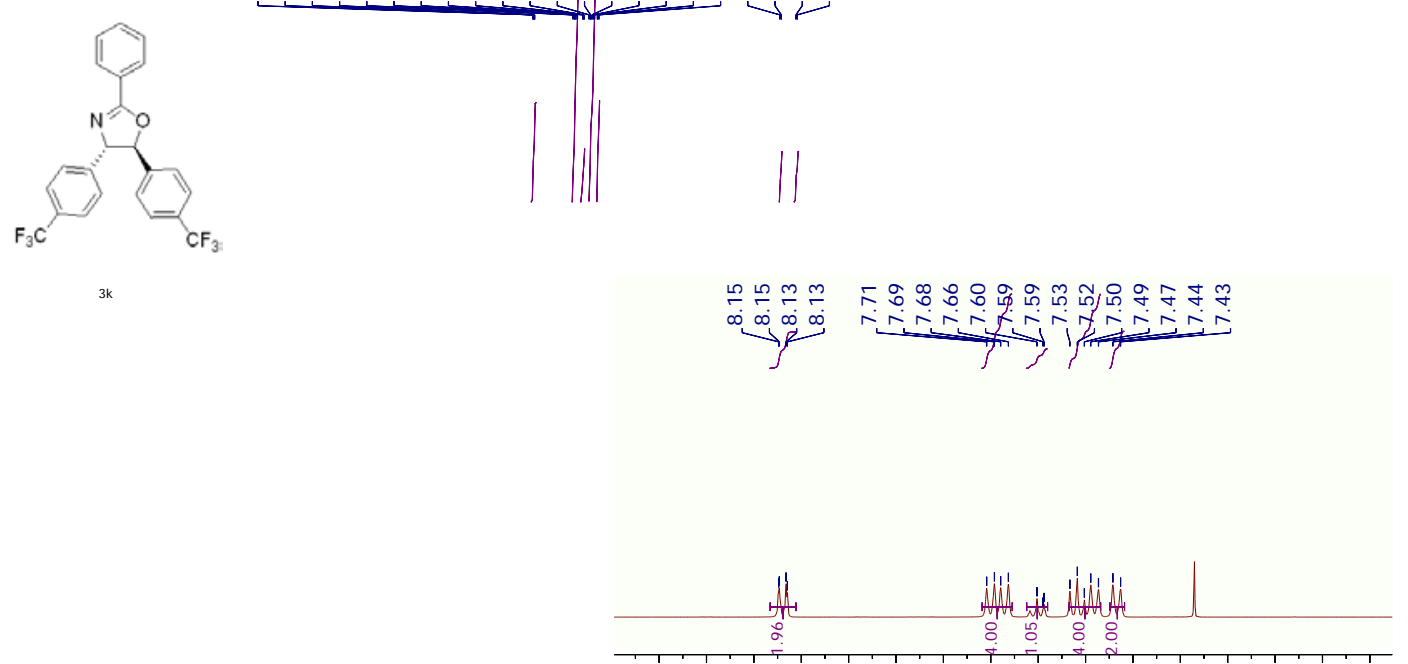

3000

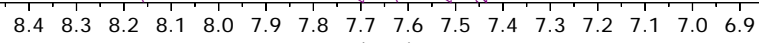

f1 (ppm)

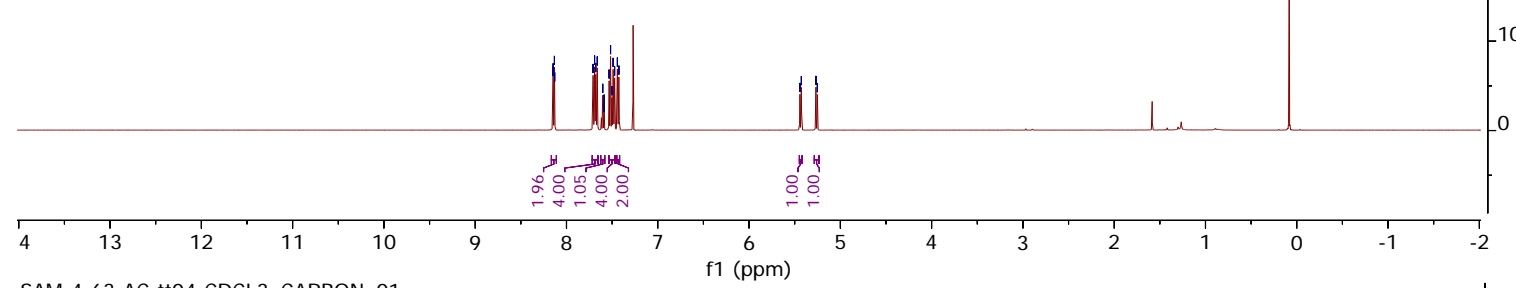

SAM-4-63-AC-tt04-CDCL3_CARBON_01

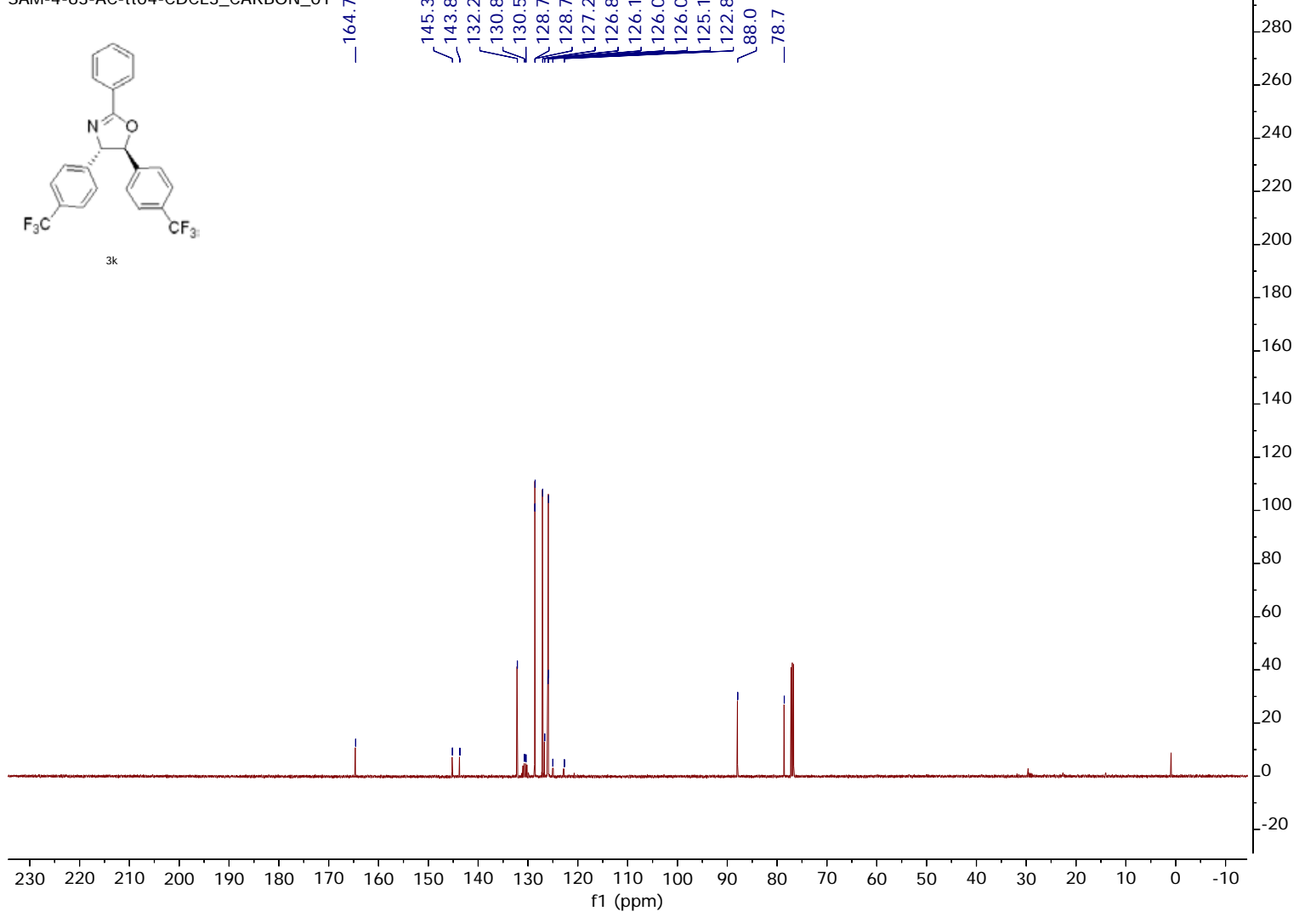




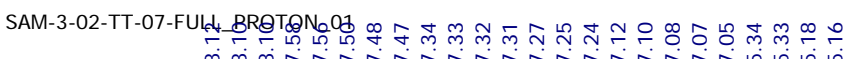

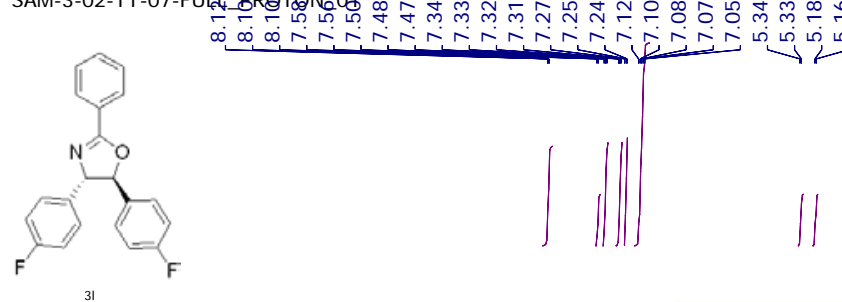

55000

50000

45000

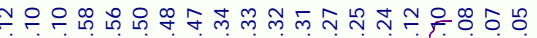

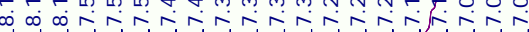

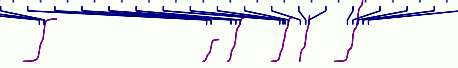

40000

35000

30000

25000

20000

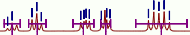

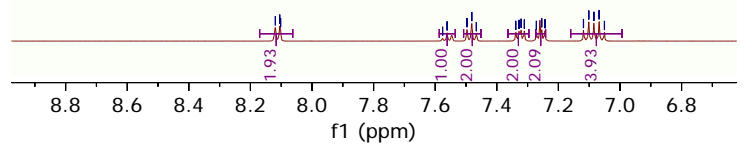

15000

10000

5000

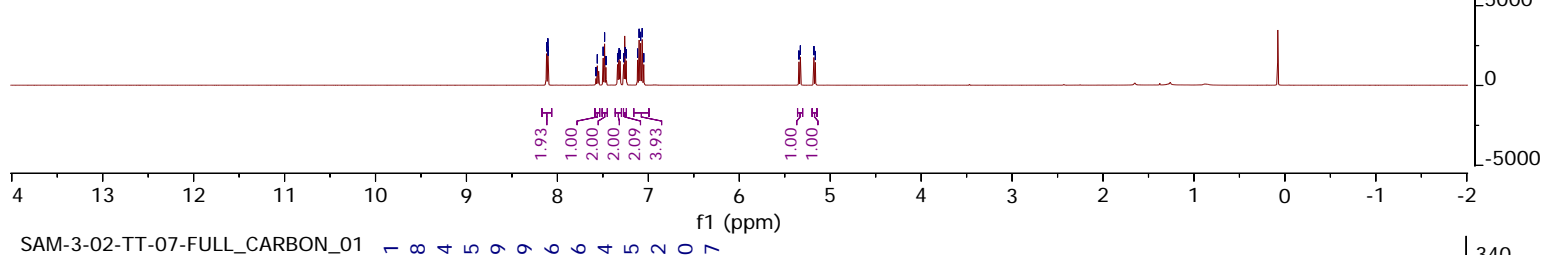

SAM-3-02-TT-07-FULL_CARBON_01 $-\infty+\infty$ ก $90000 \%$ f1 (ppm)

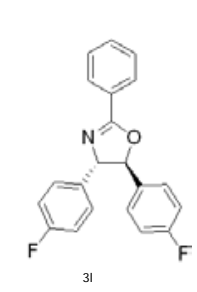

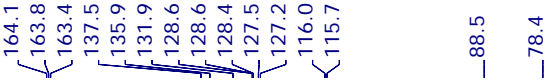

340

320

300

280

260

240

220

200

180

160

140

120

100

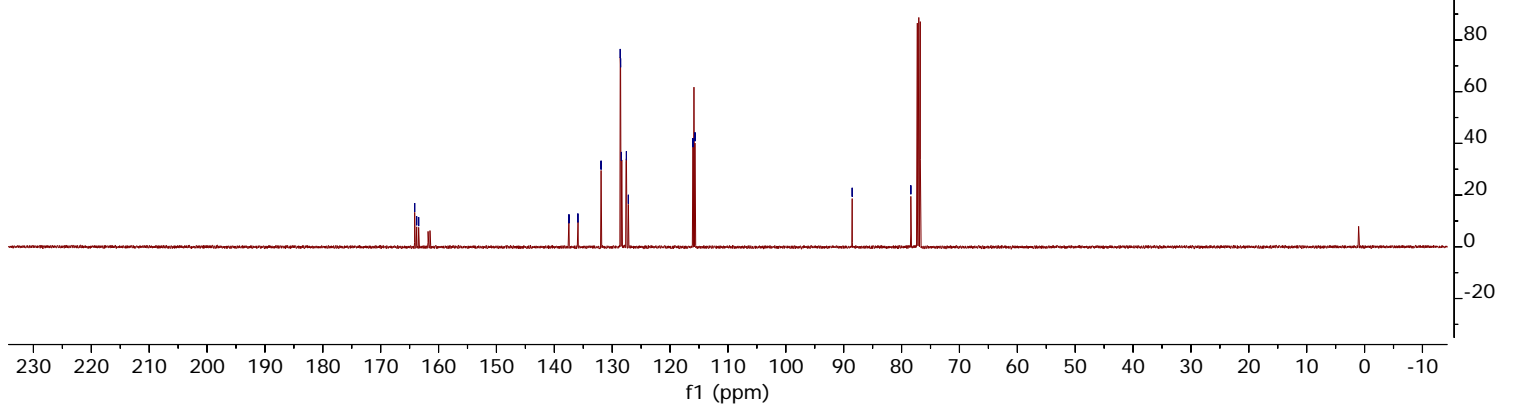




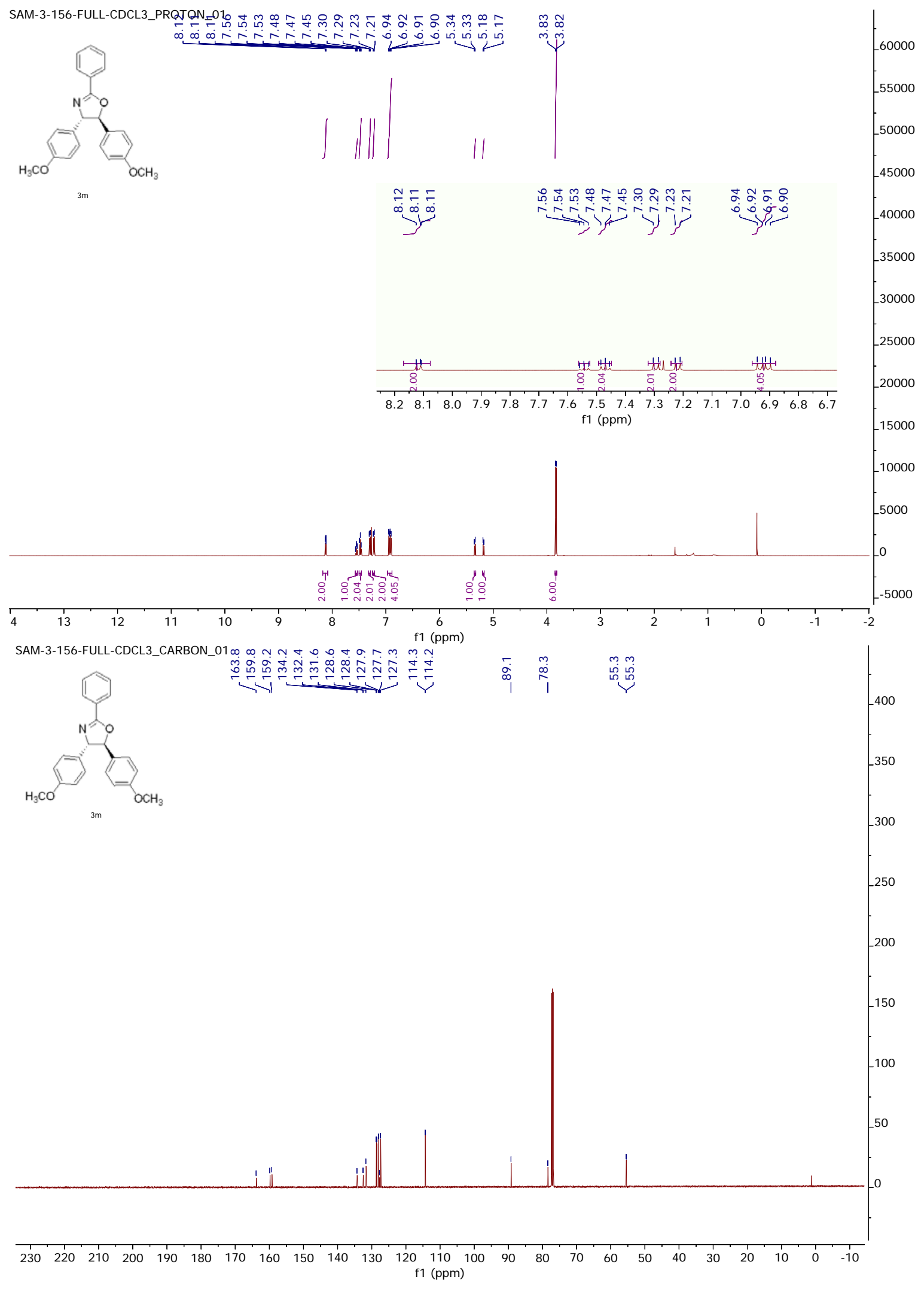




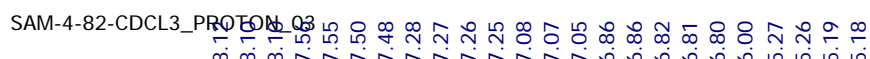

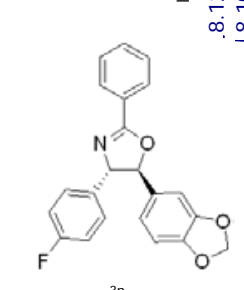

虽

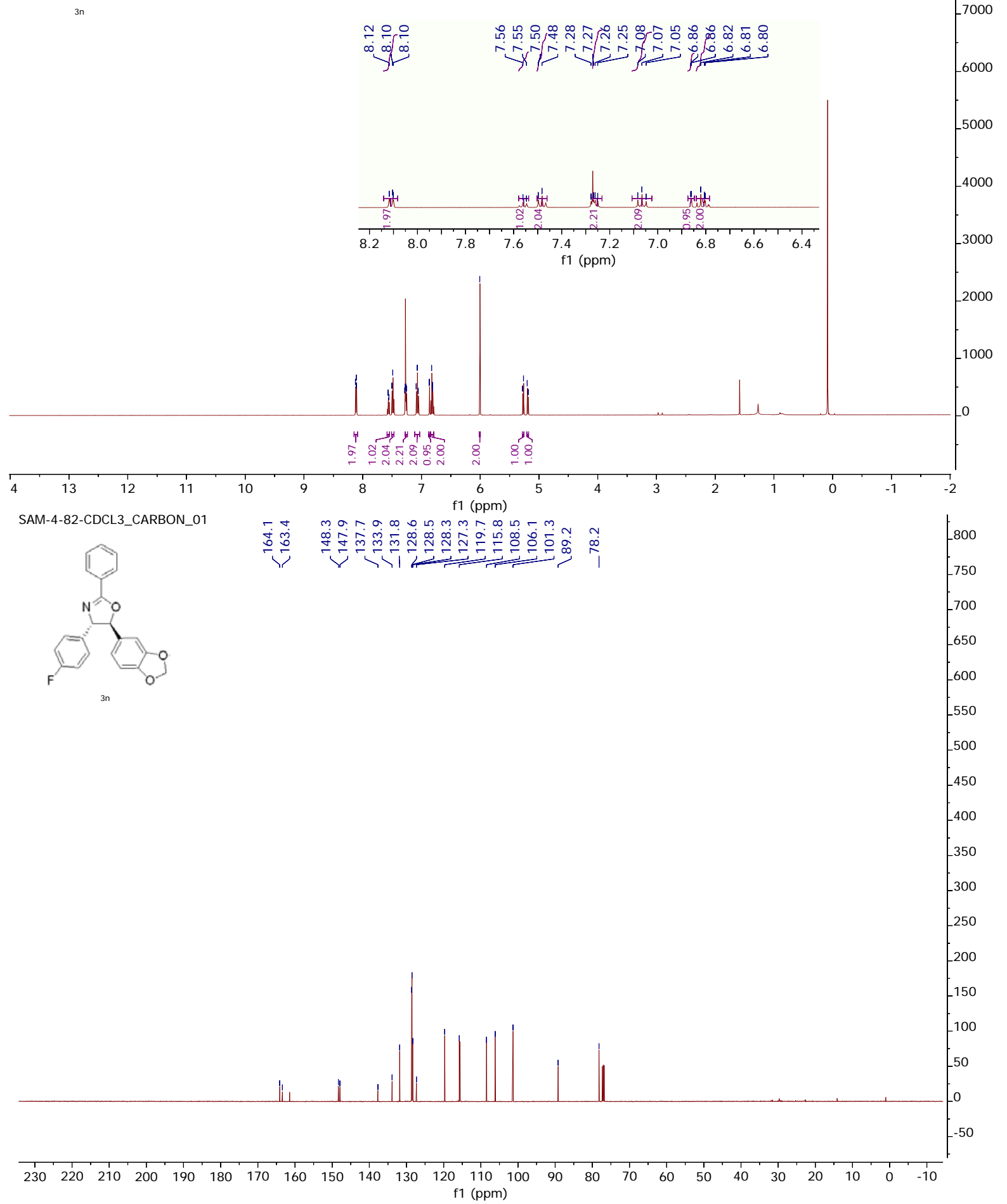




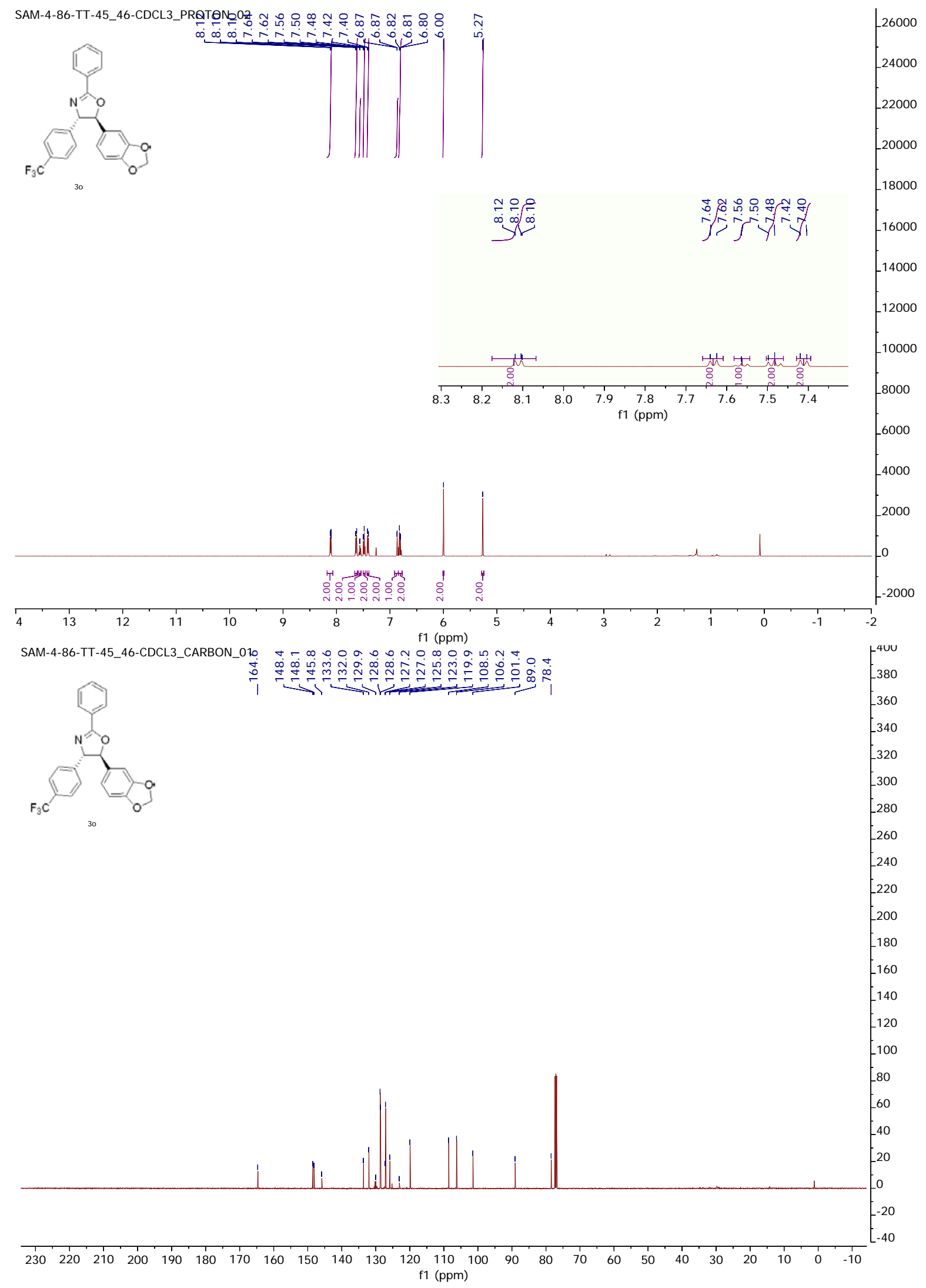



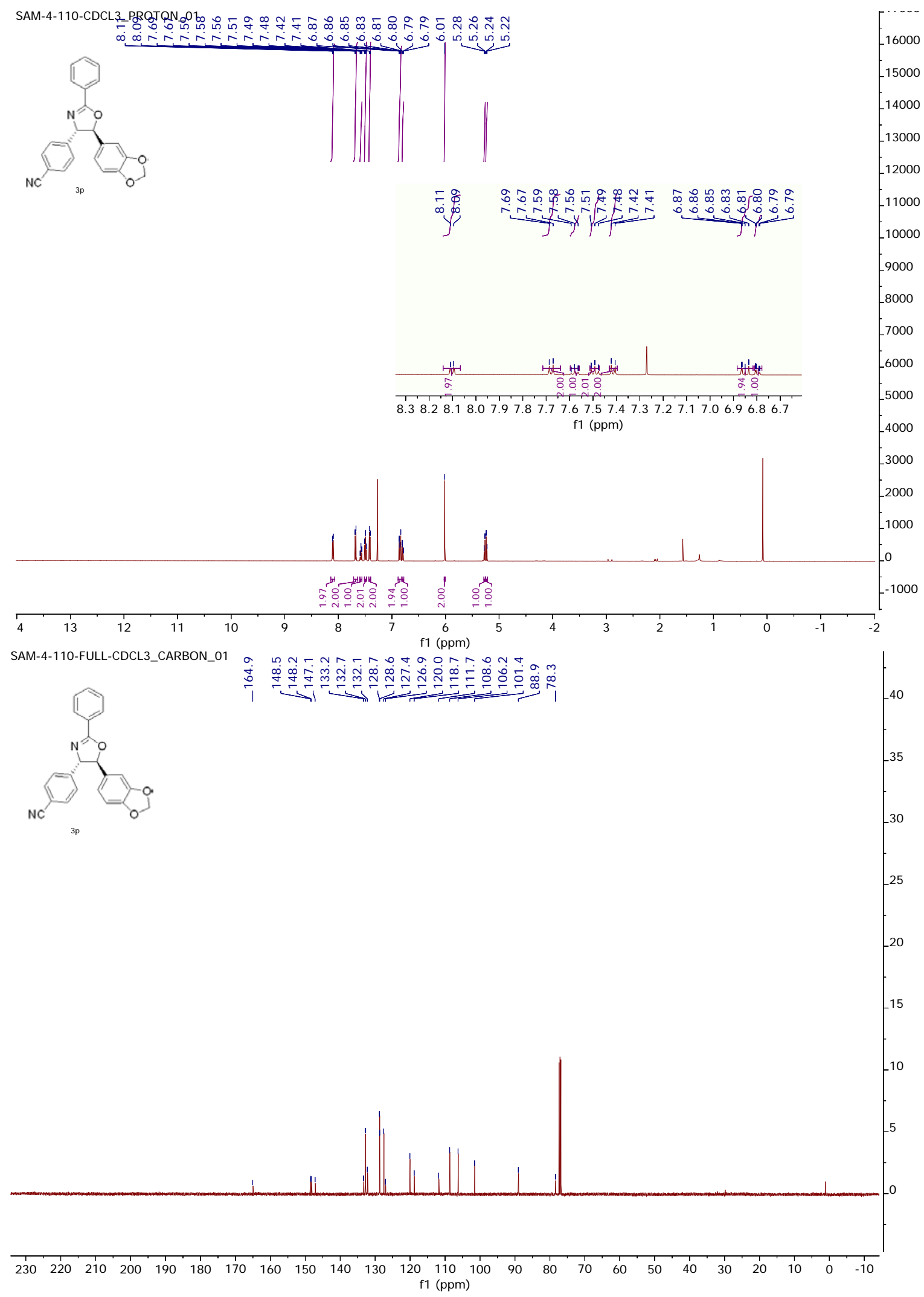


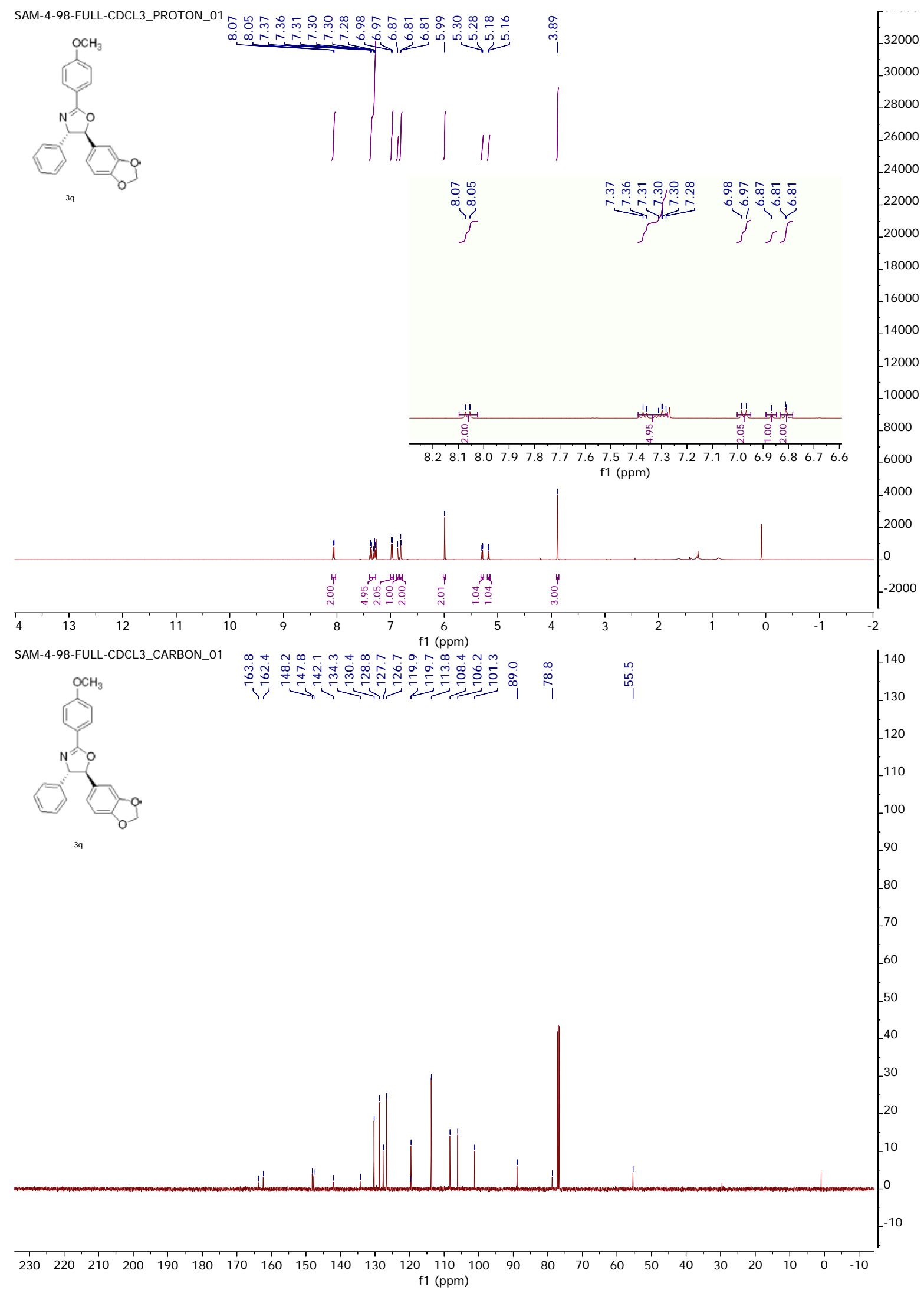


SAM-4-99-AC-TT-53-CDCL3_PROTON⿴囗大)
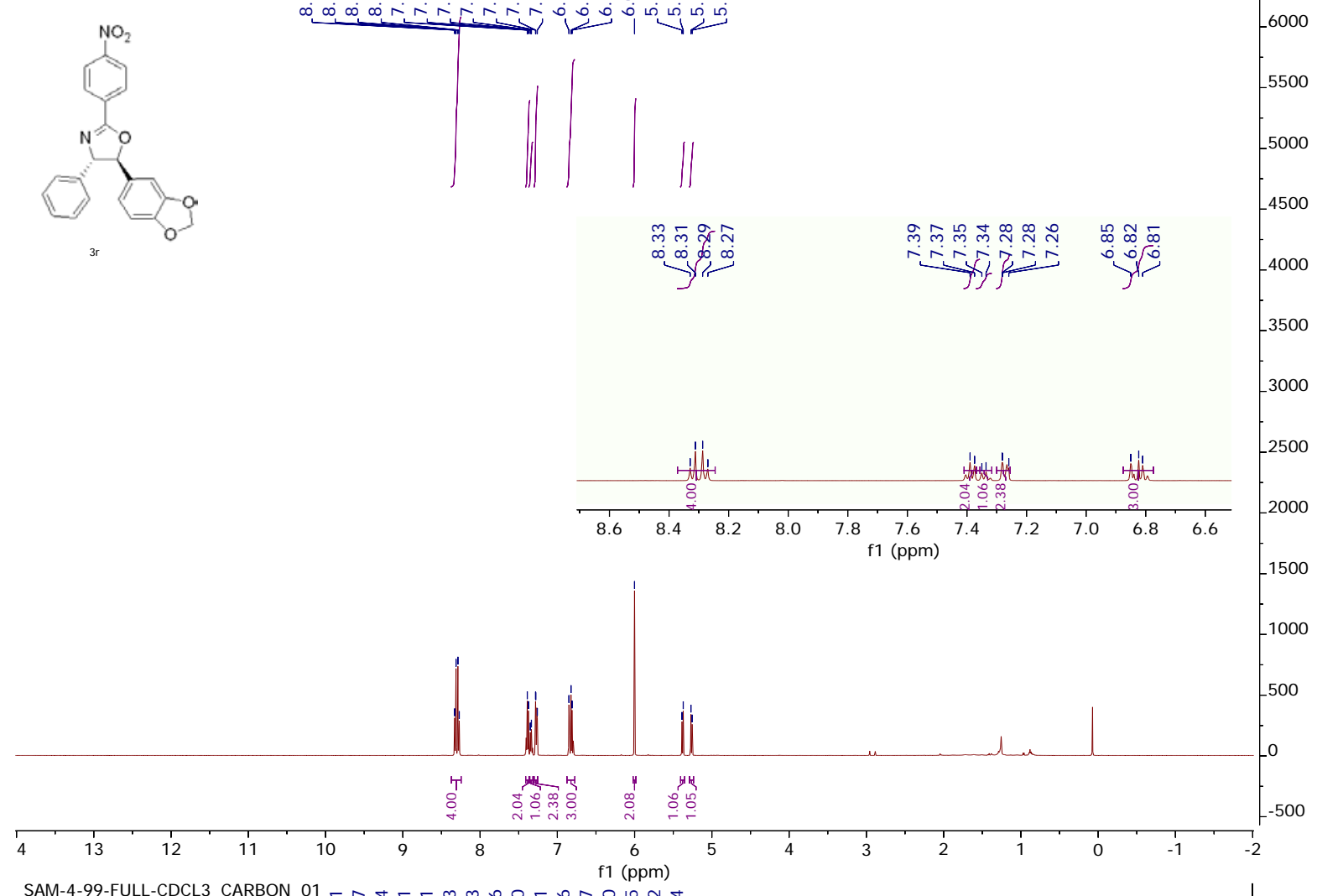
SAM-4-99-FULL-CDCL3_CARBON_01 H A-4
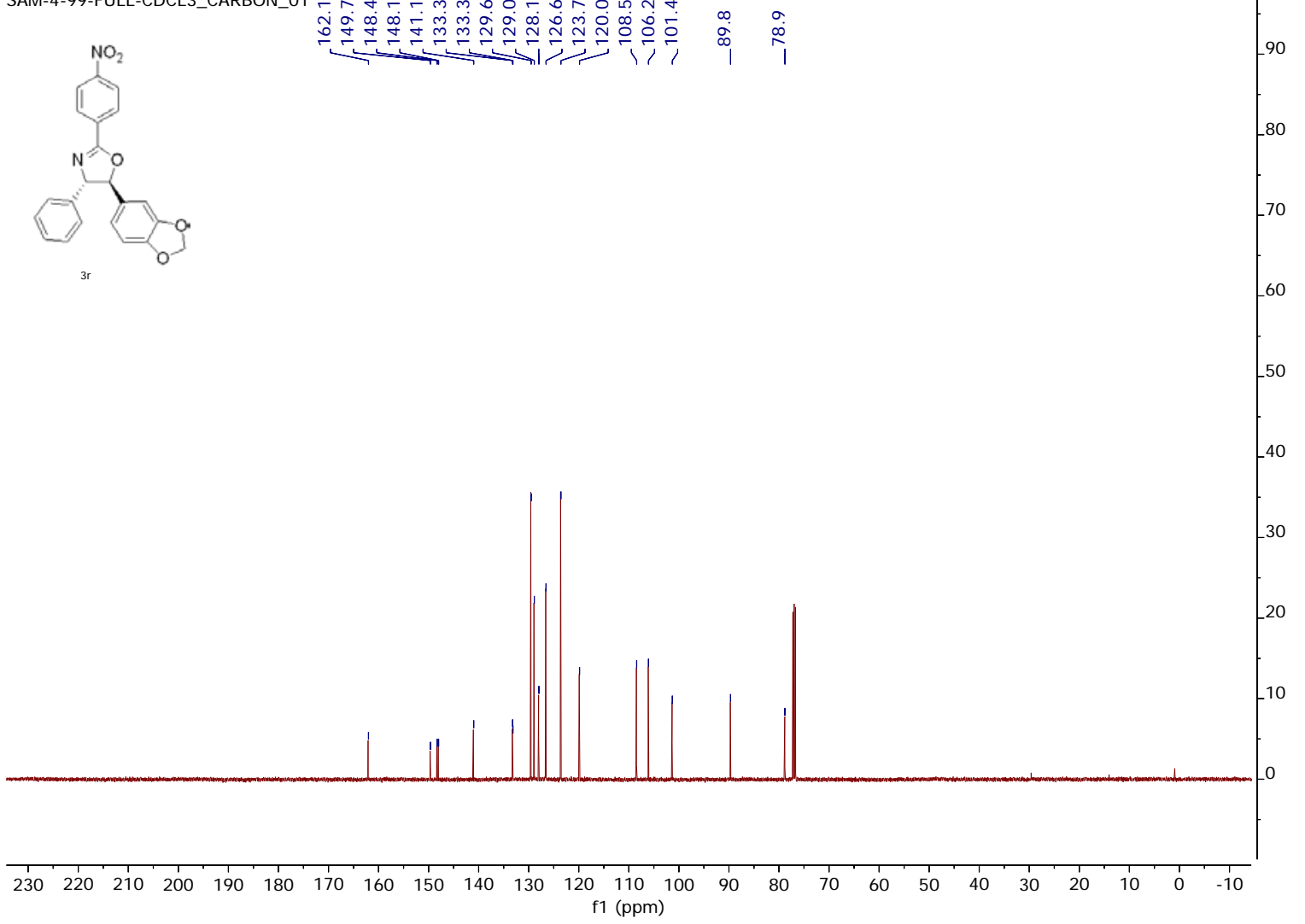


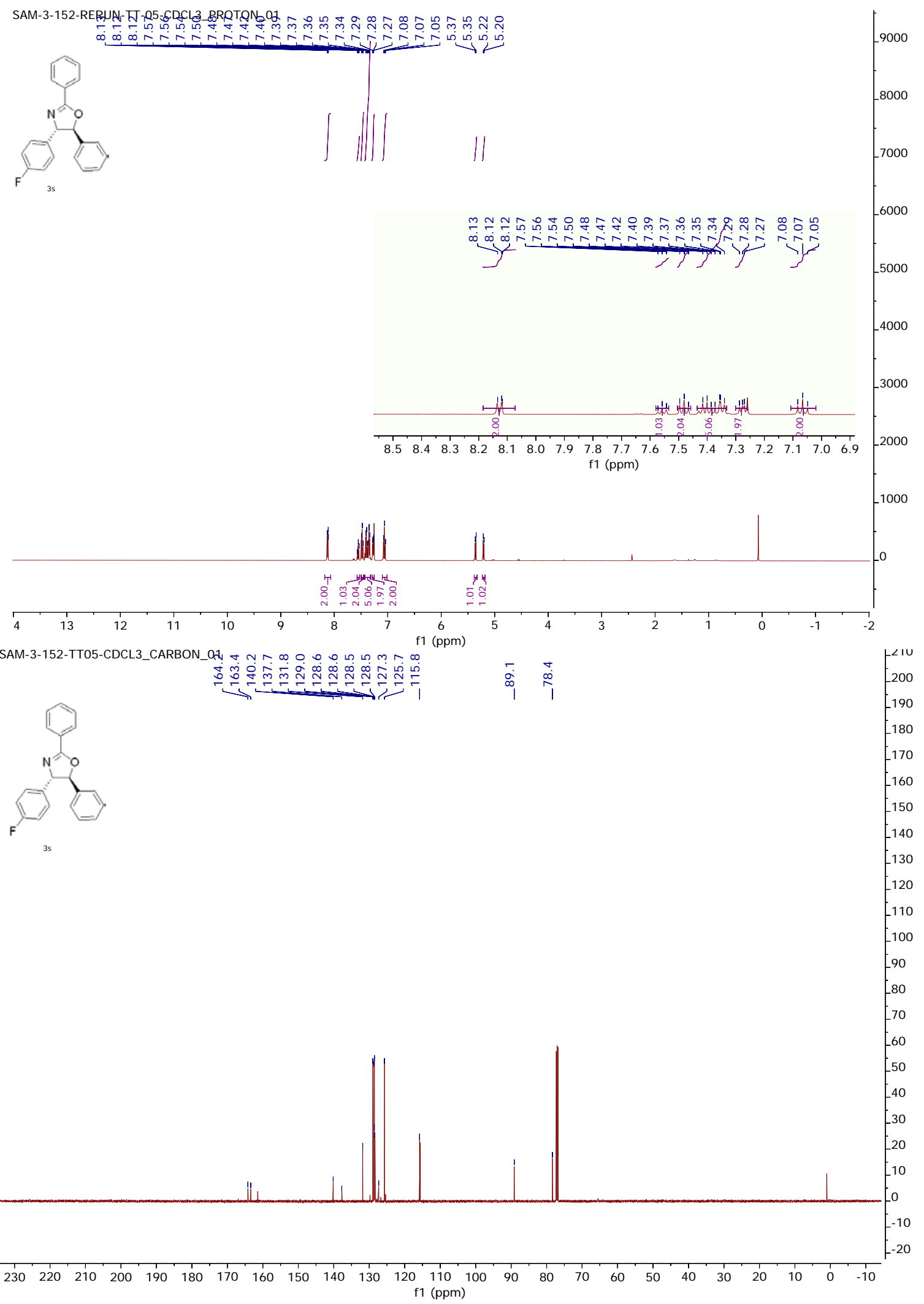




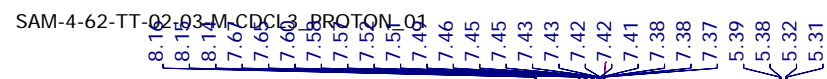
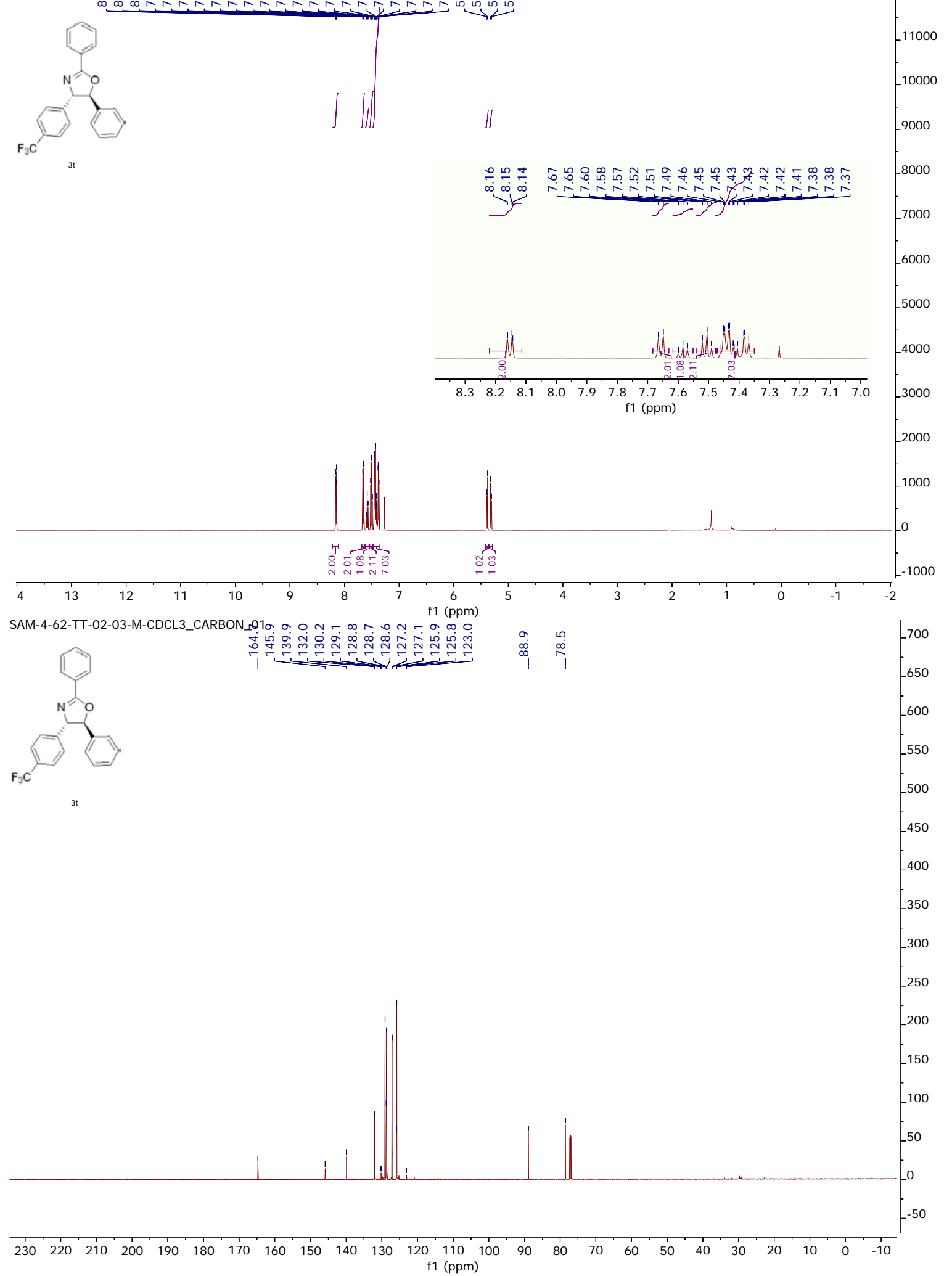


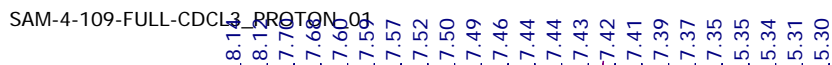

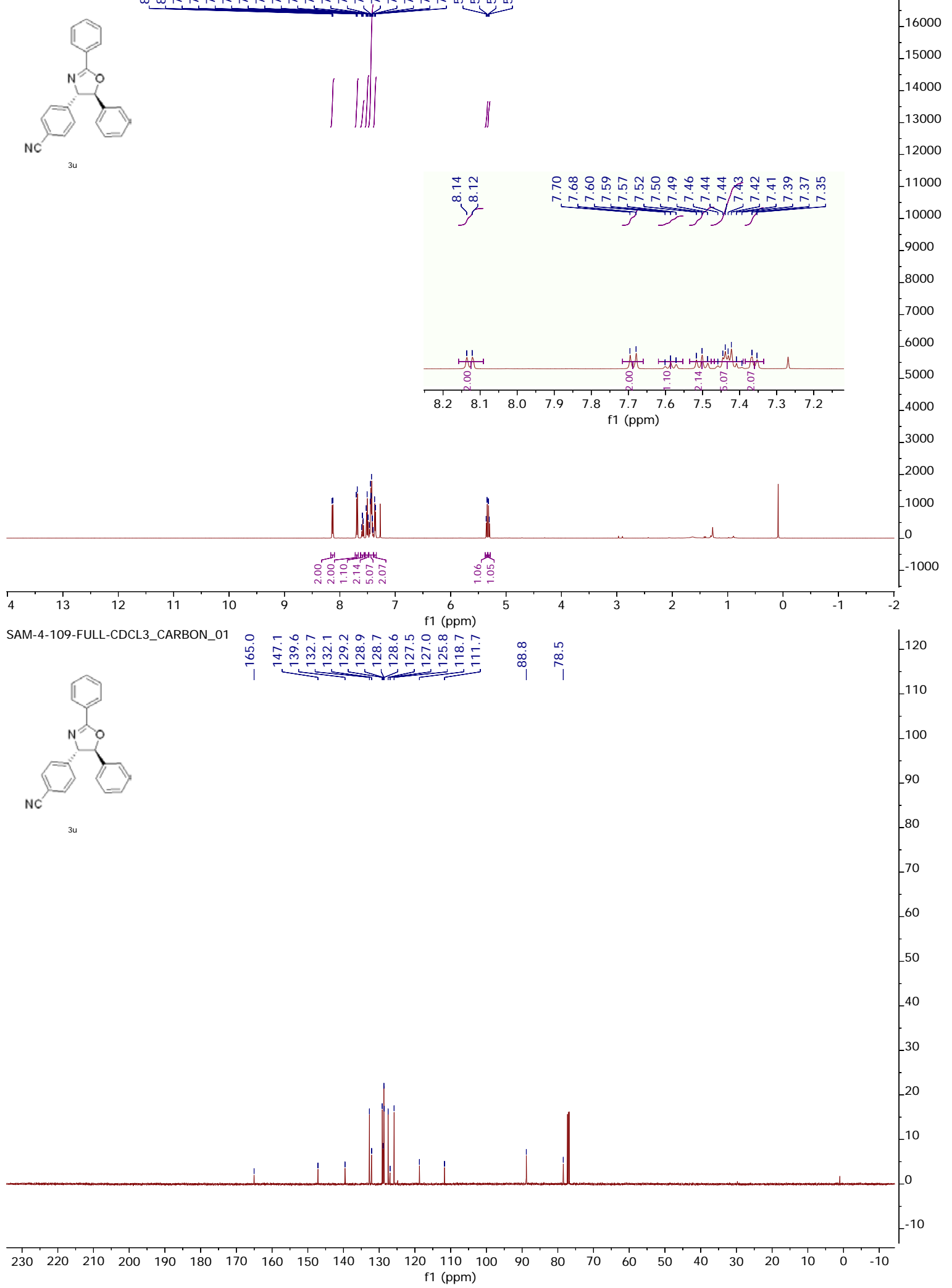




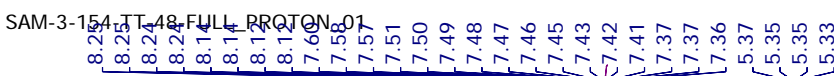
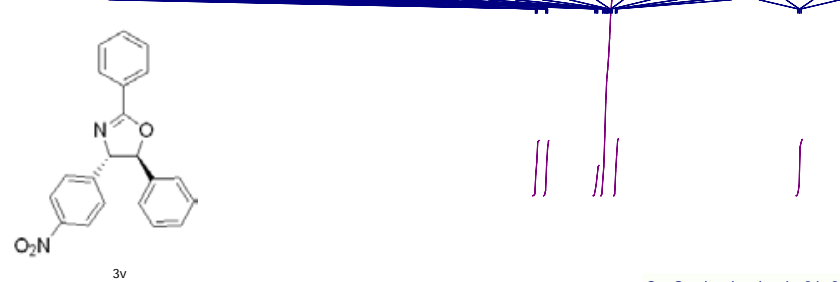

26000

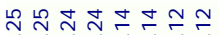

$\infty$

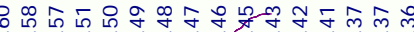

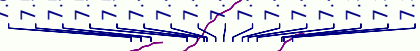

16000

14000

12000

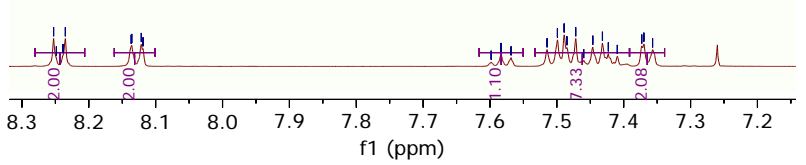

10000

8000

6000

4000

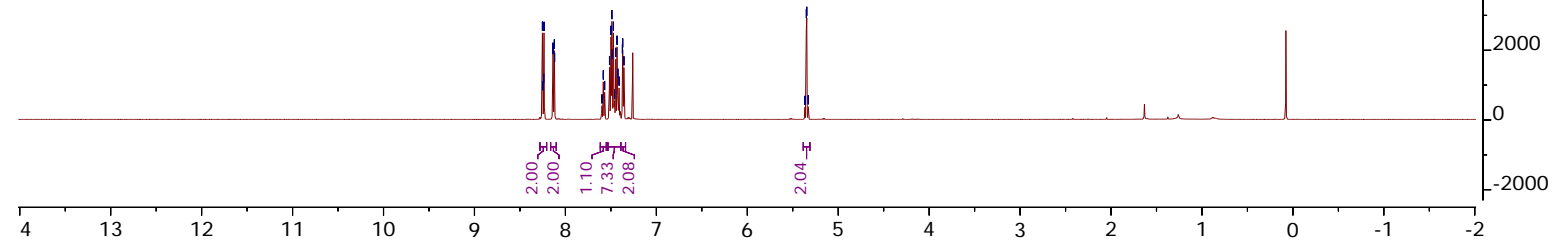

$\begin{array}{llllll}4 & 13 & 12 & 11 & 10 & 9\end{array}$

SAM-3-154-TT-48-FULL_CARBON_01
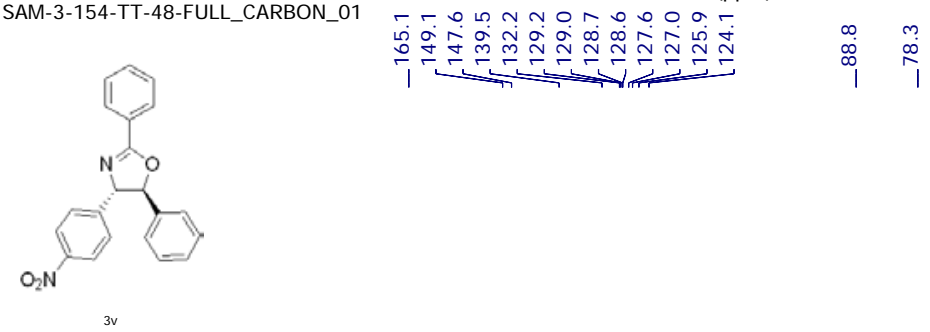

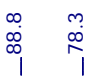

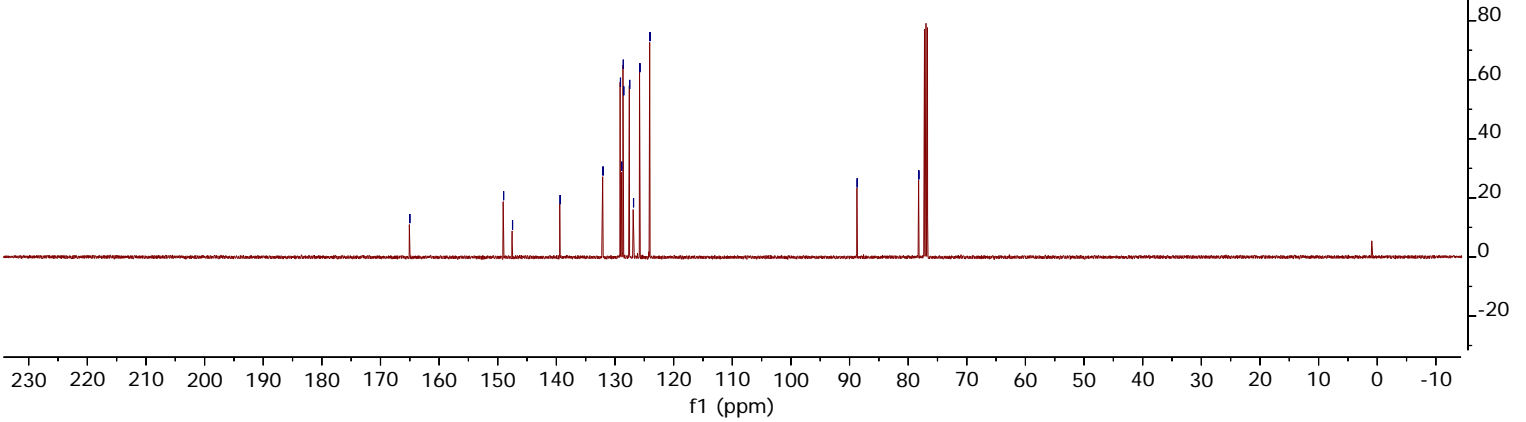




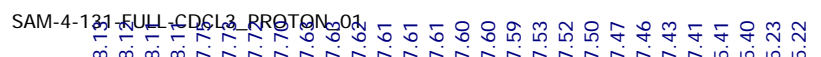
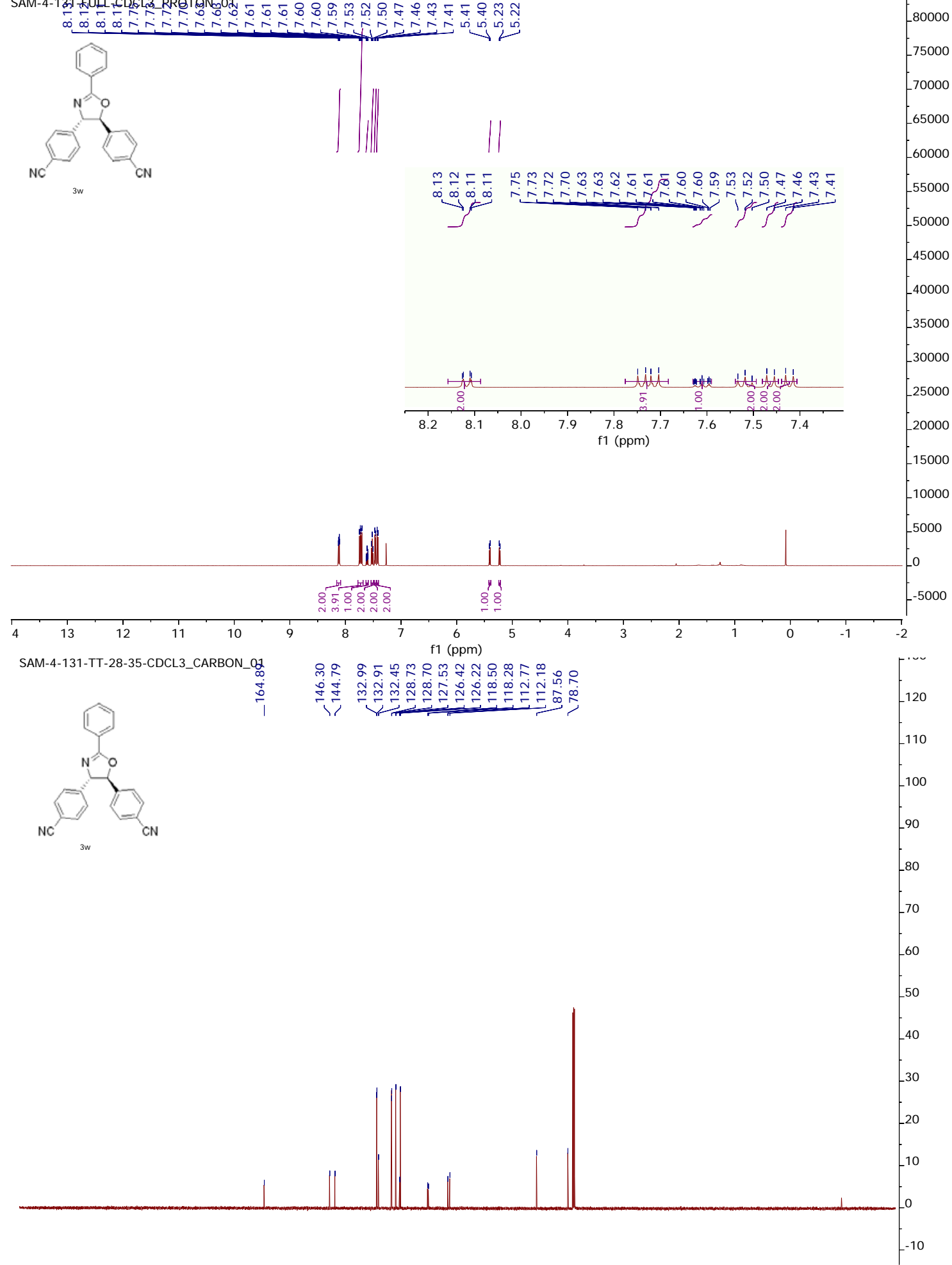

$\begin{array}{lllllllllllllllllllllllllllll}230 & 220 & 210 & 200 & 190 & 180 & 170 & 160 & 150 & 140 & 130 & 120 & 110 & 100 & 90 & 80 & 70 & 60 & 50 & 40 & 30 & 20 & 10 & 0 & -10\end{array}$ 


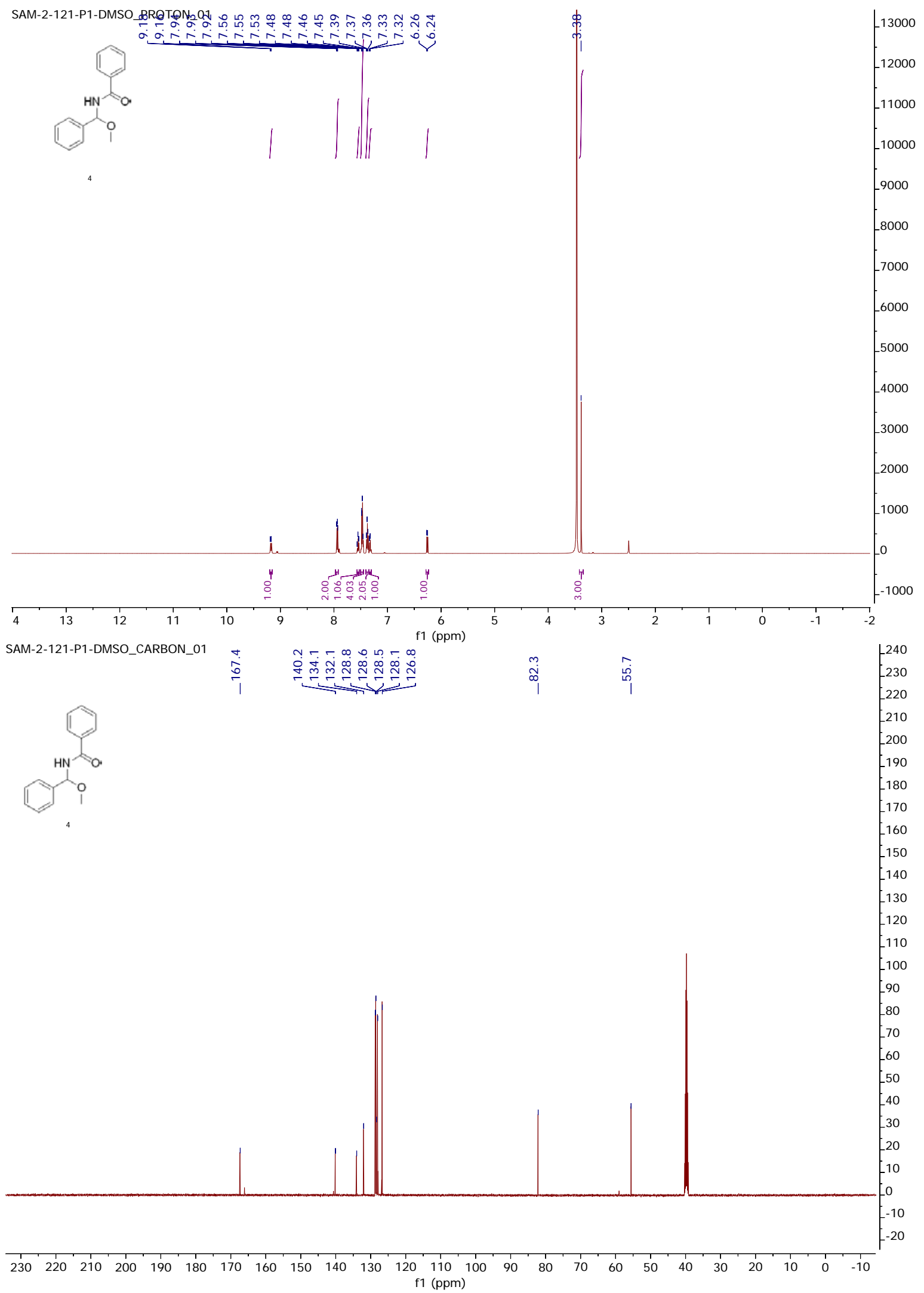




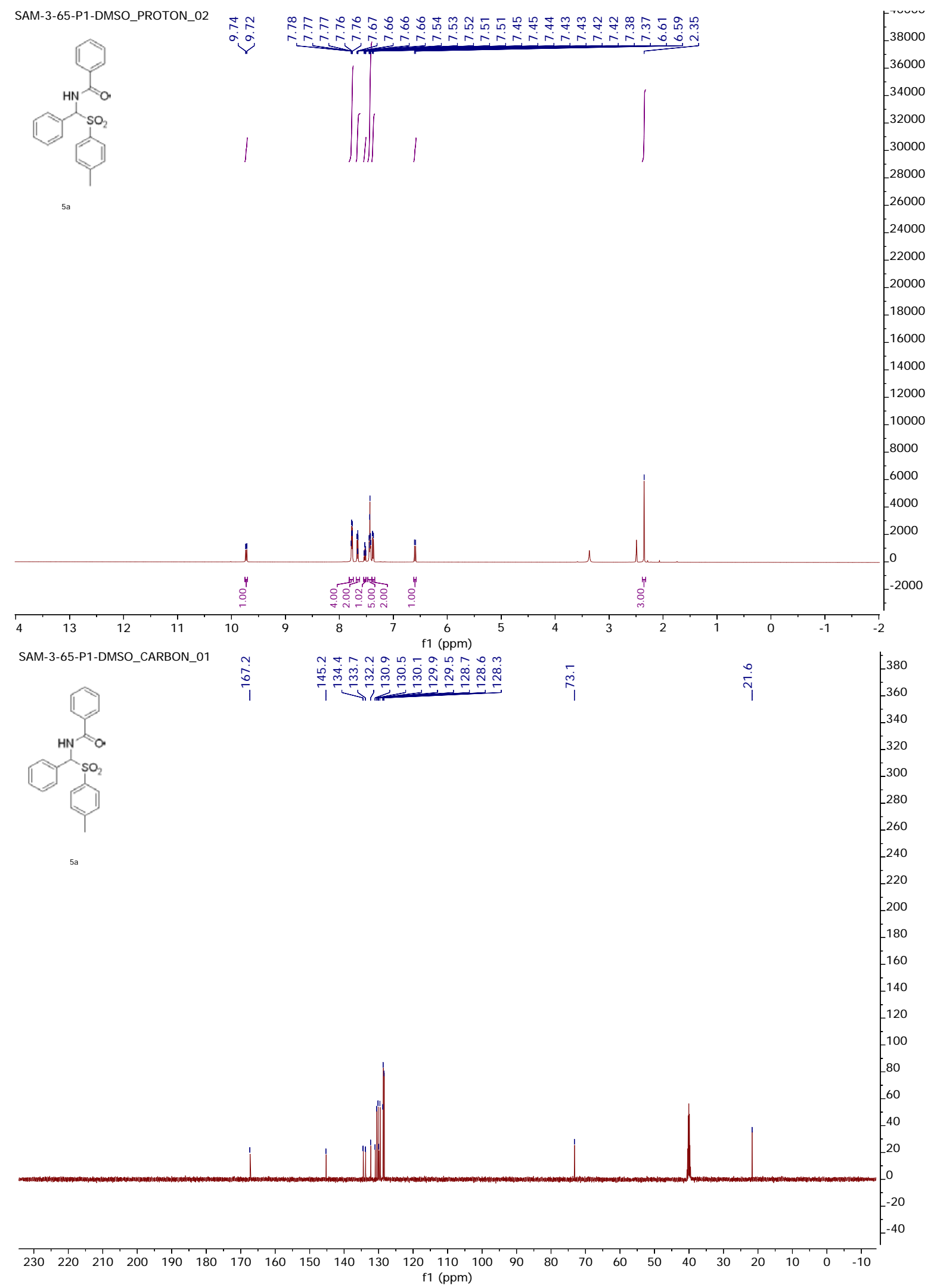




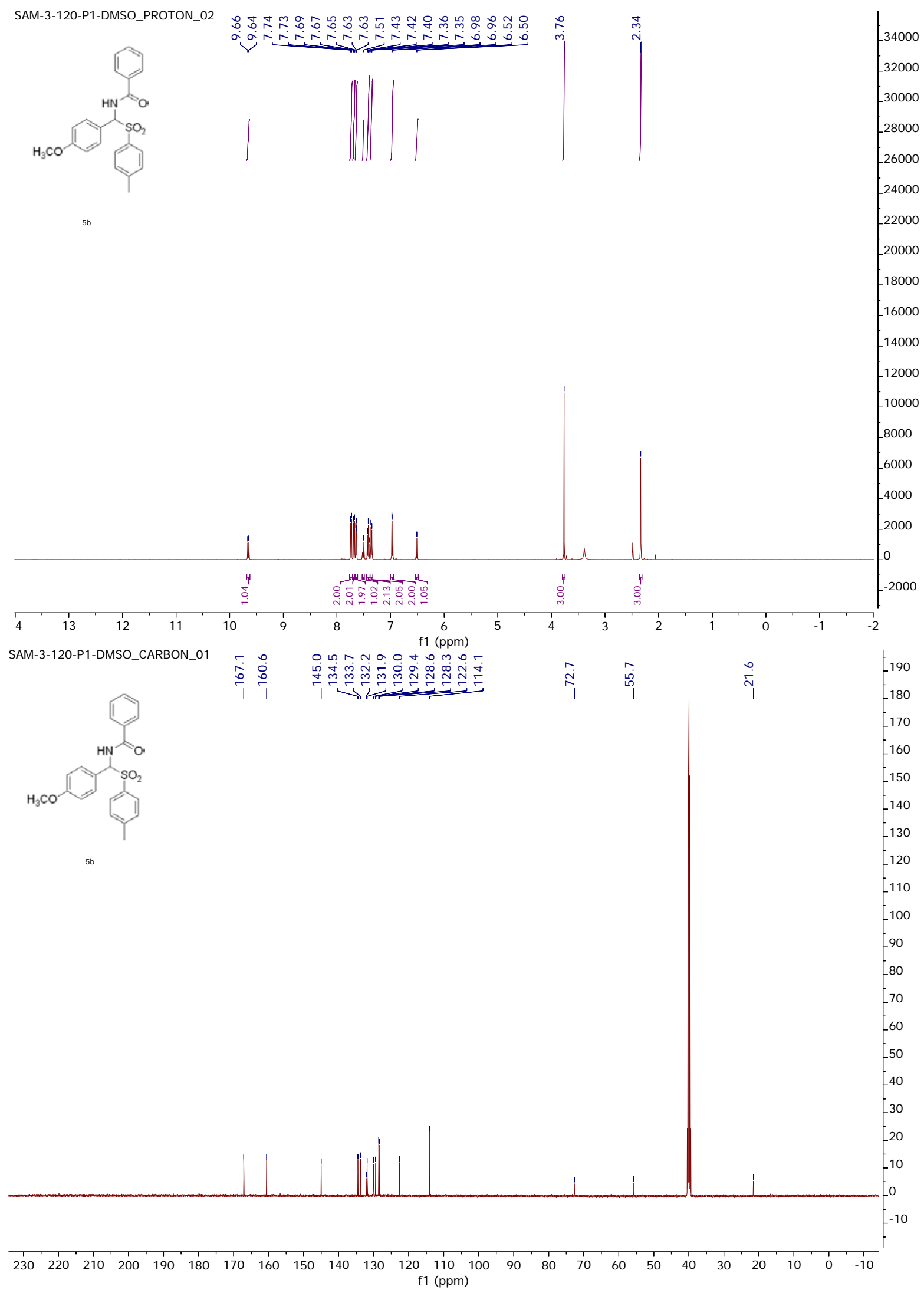




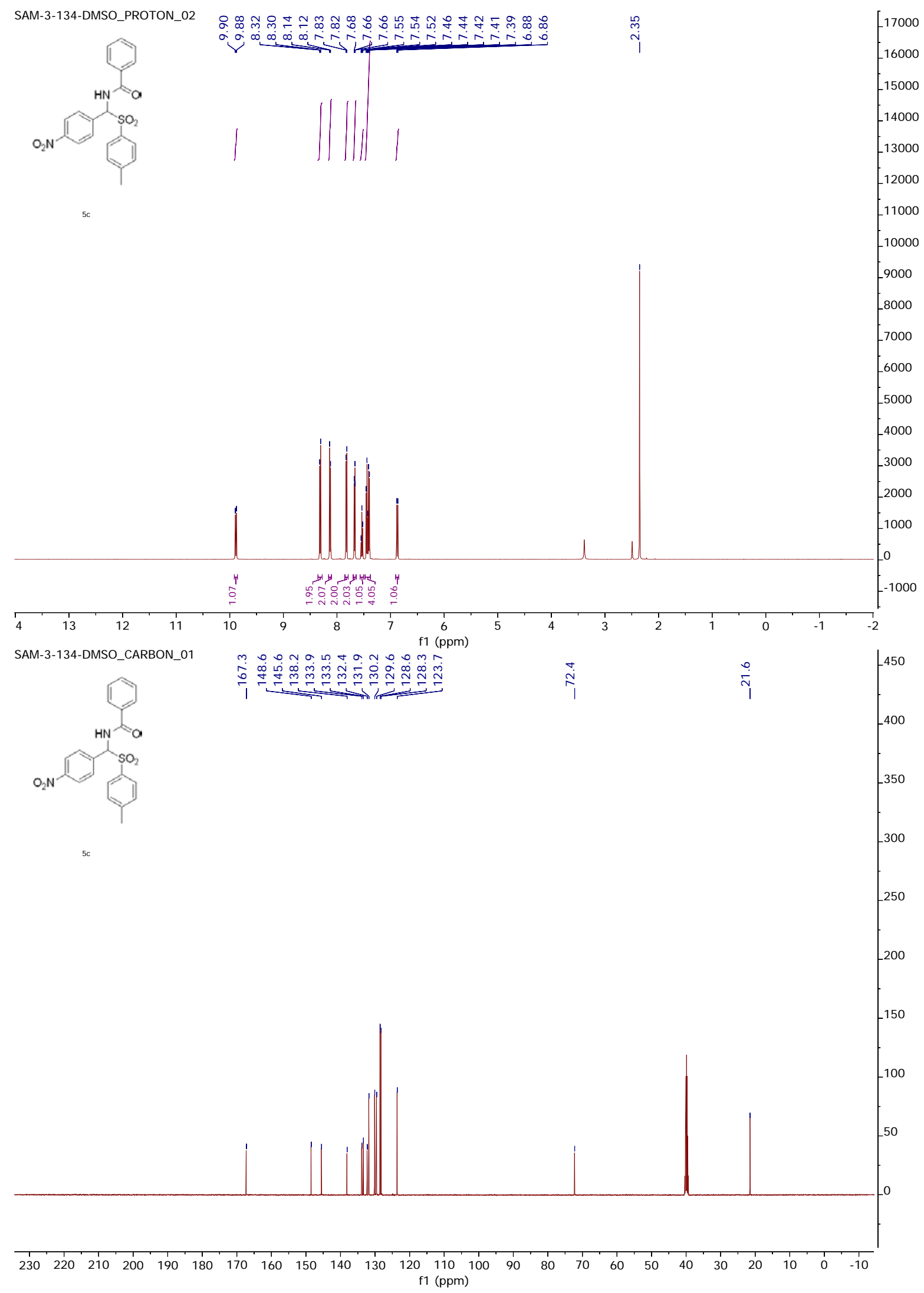



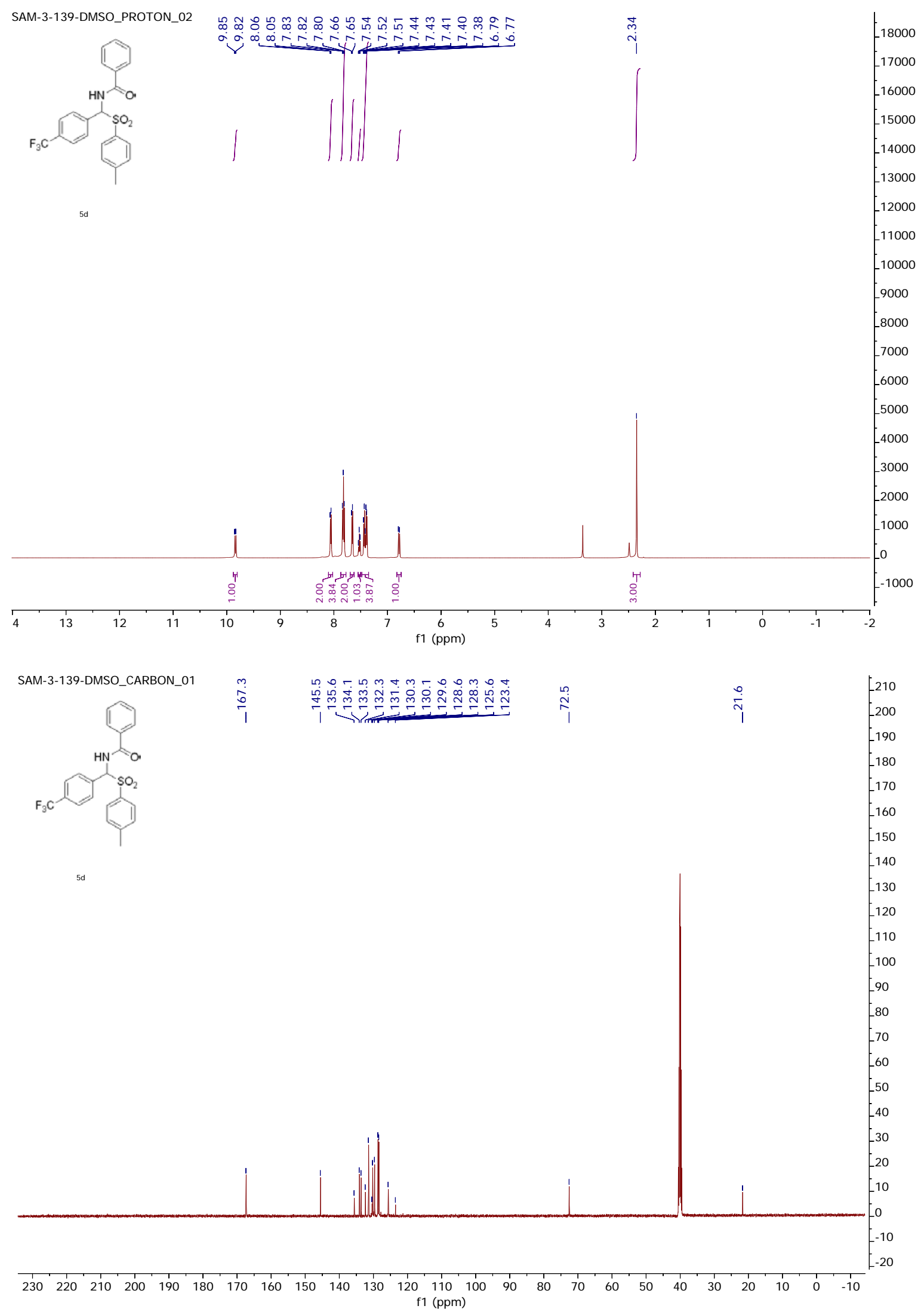

S51 


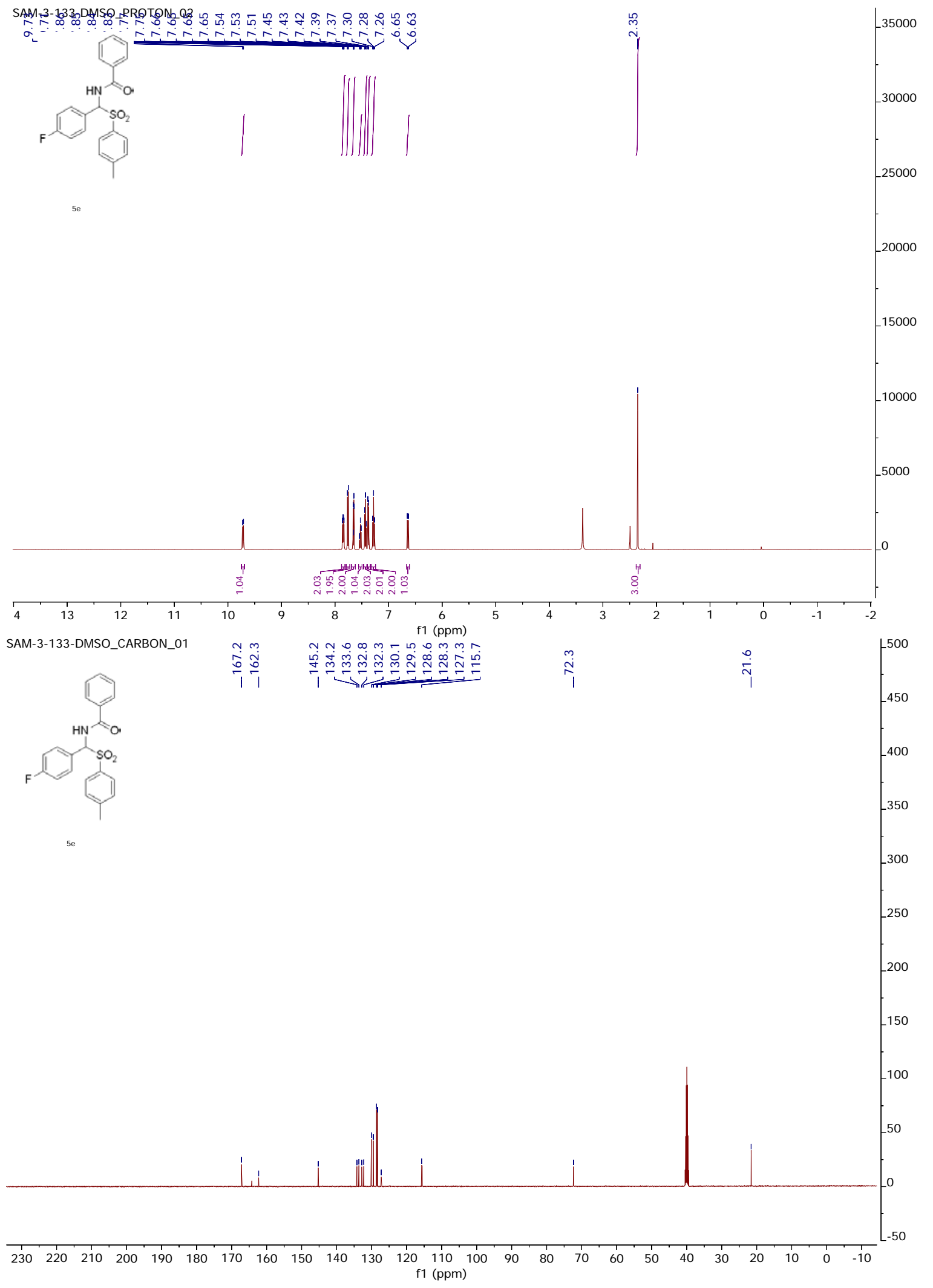




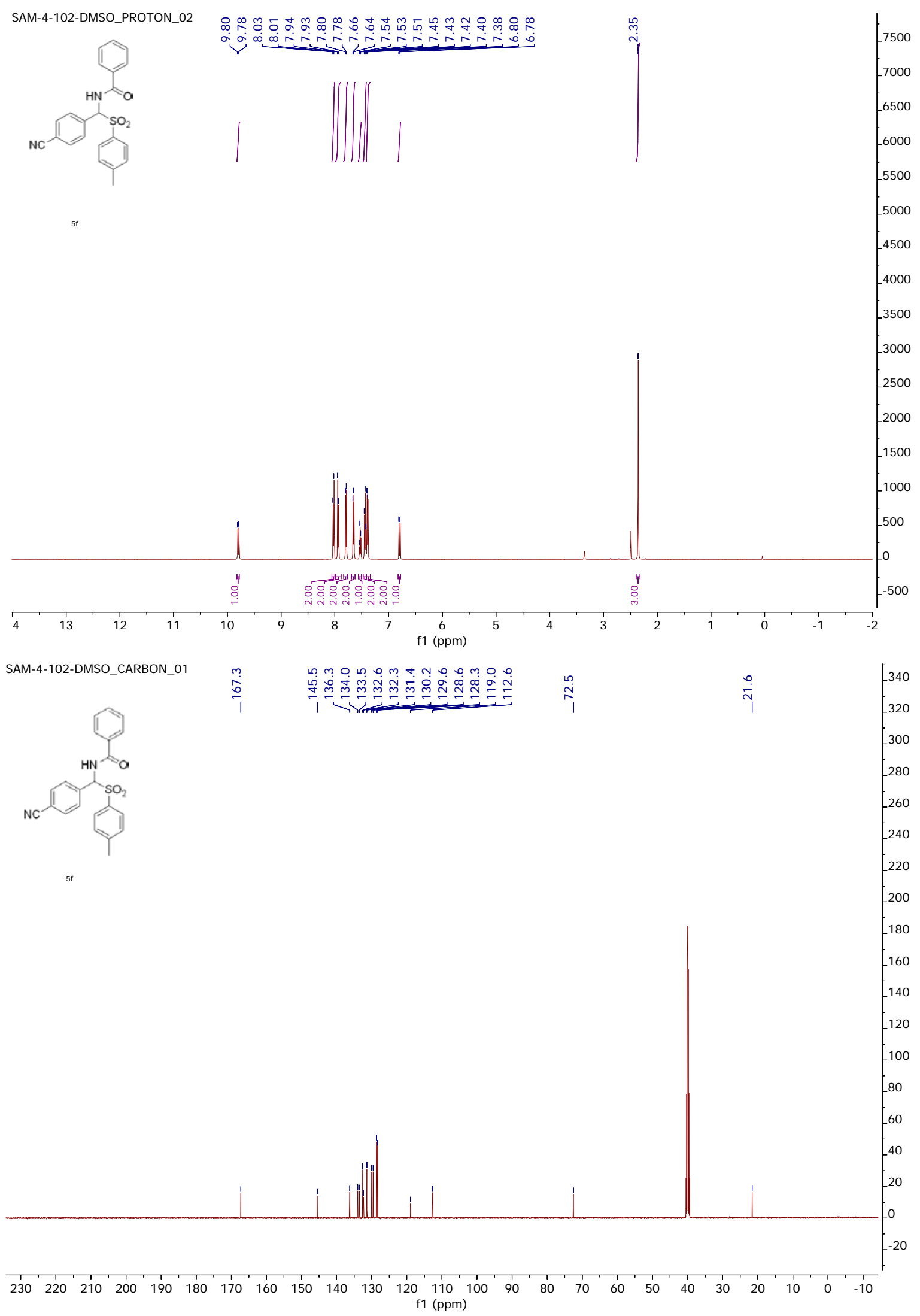




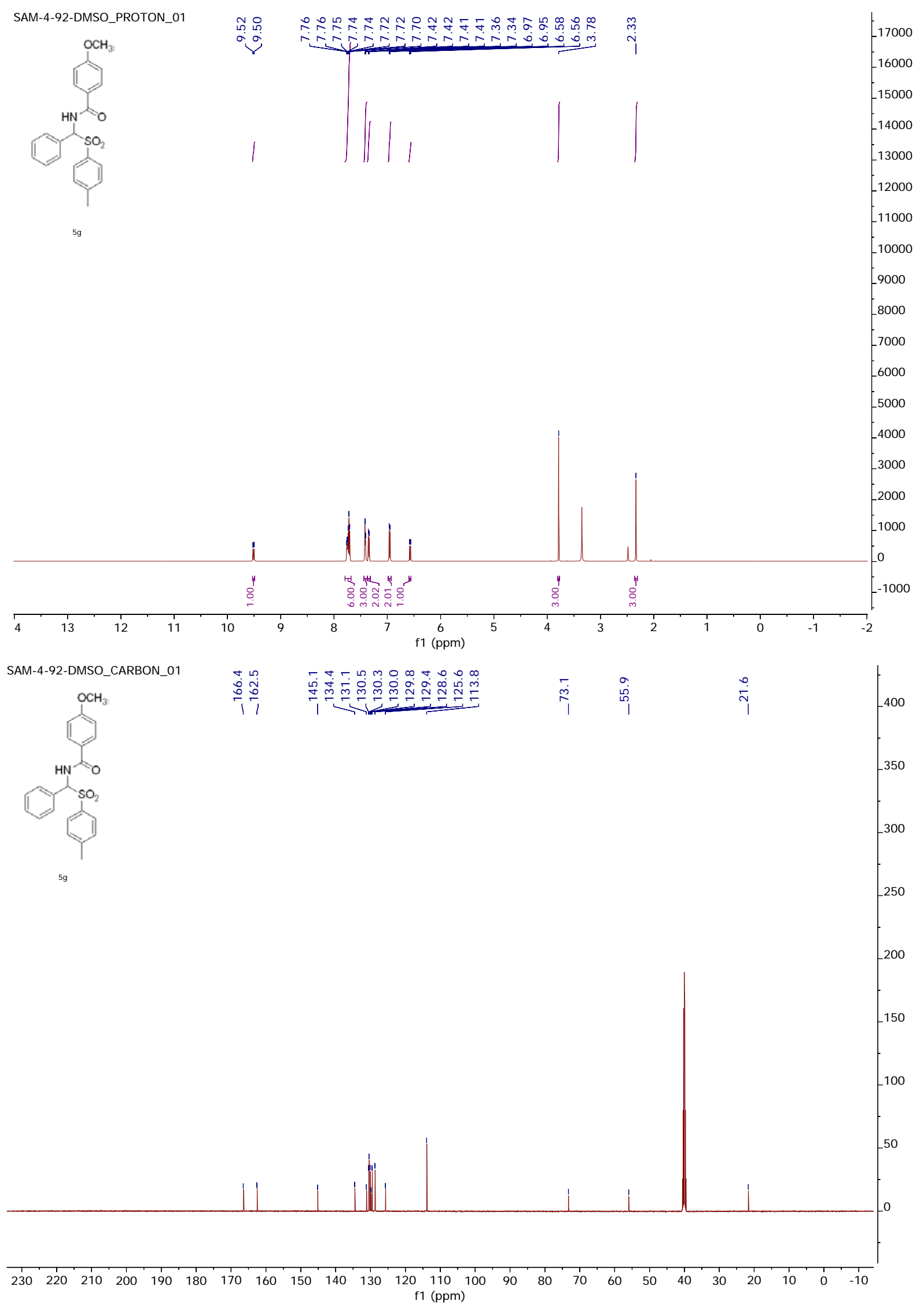




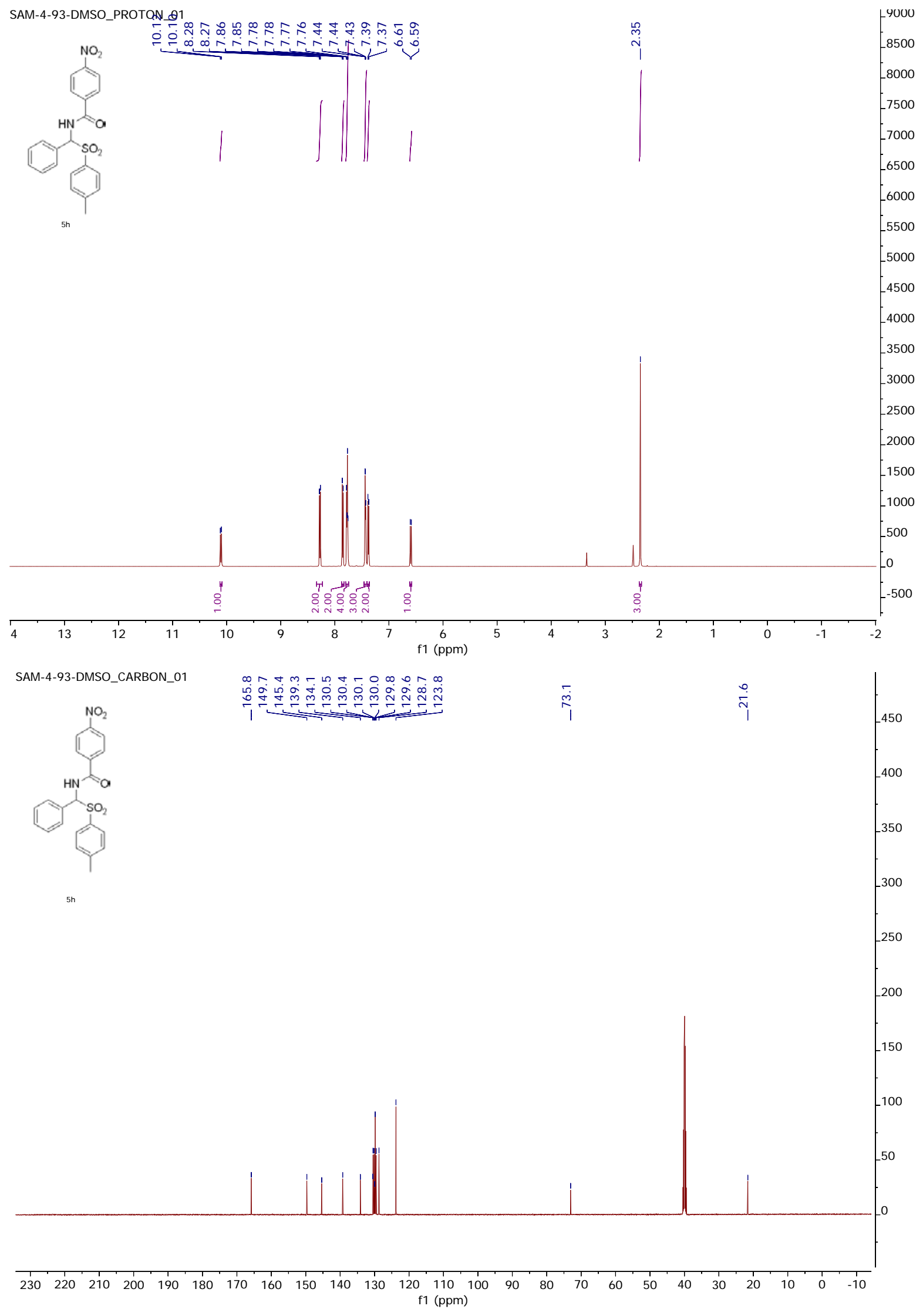




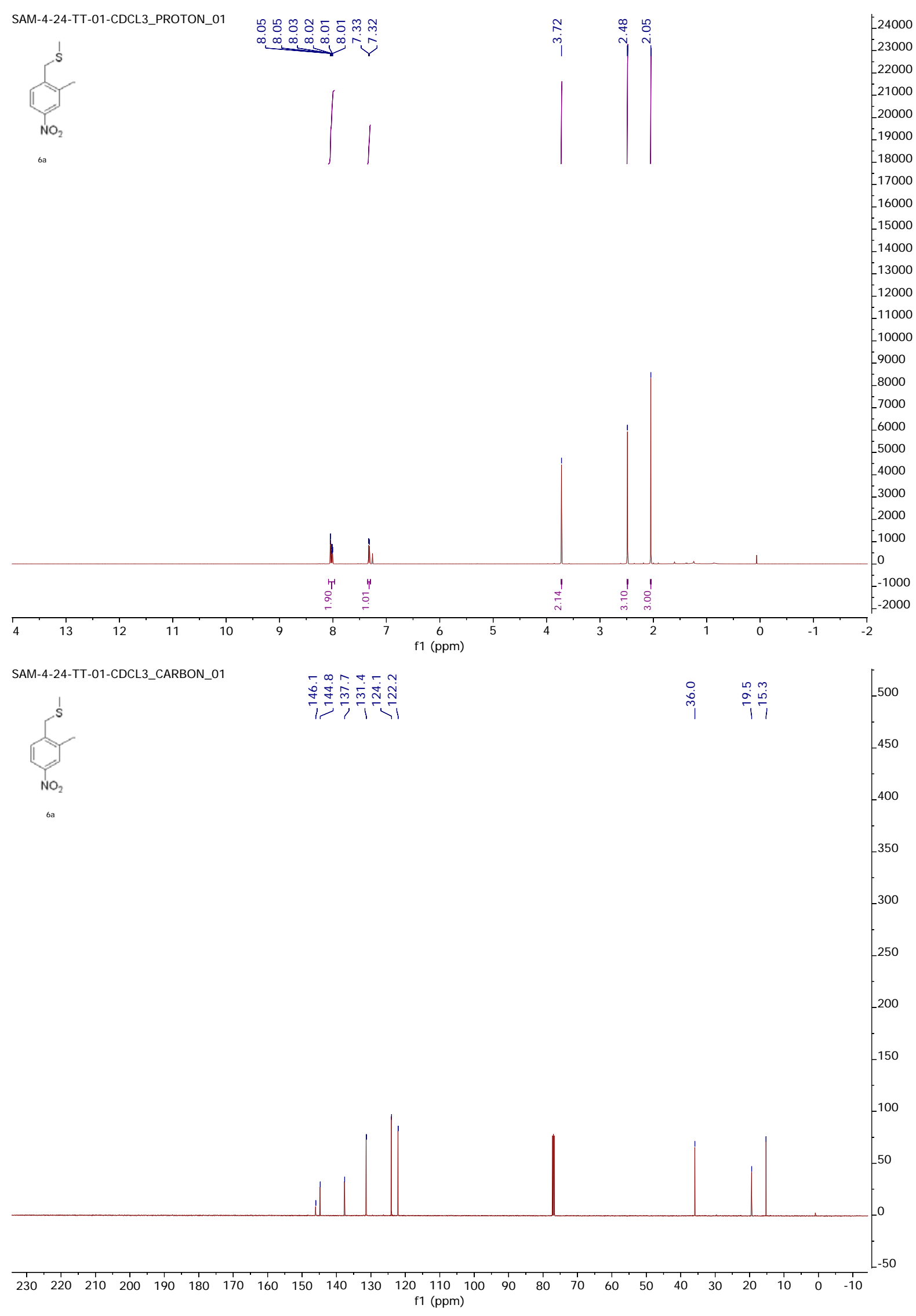




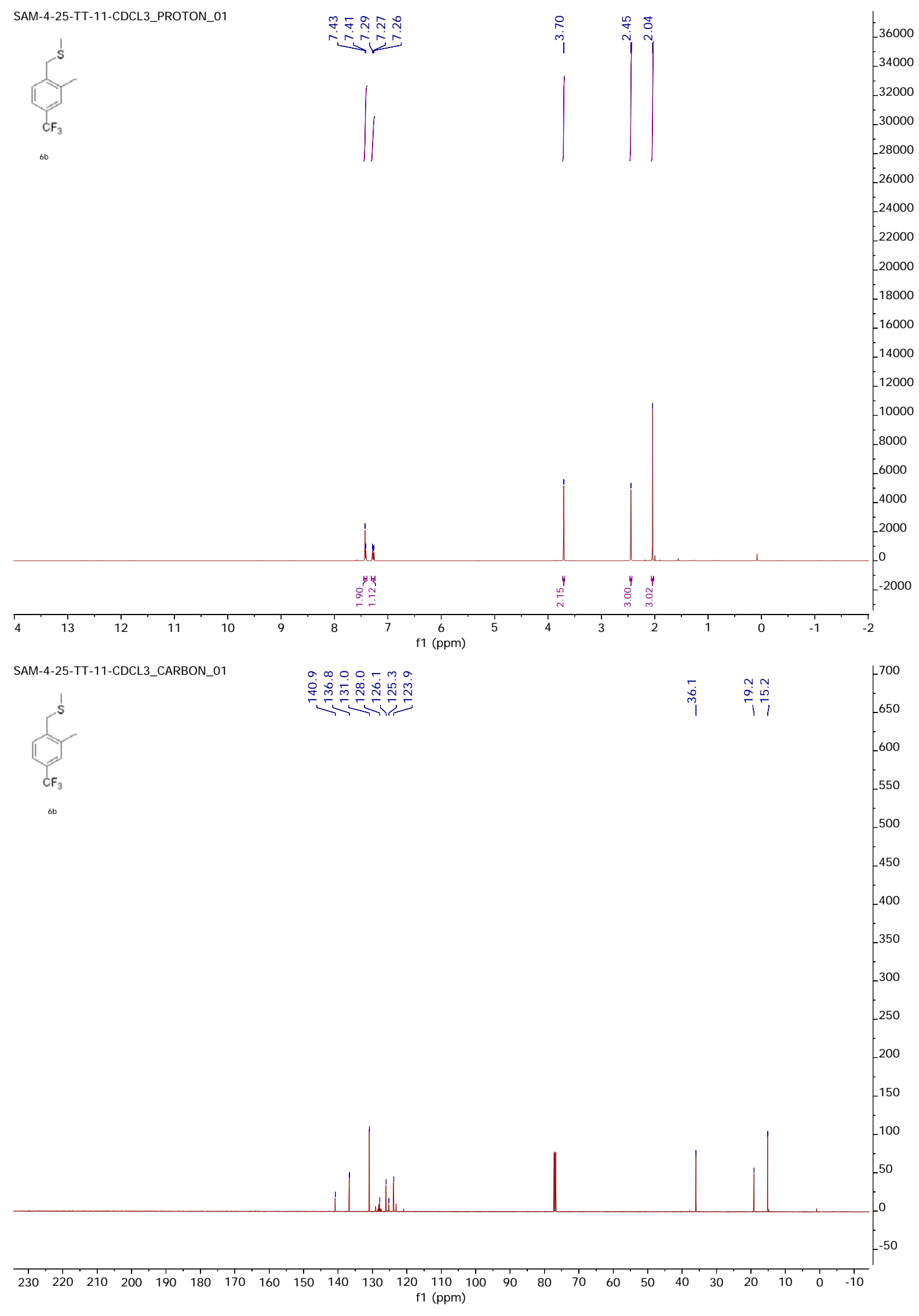




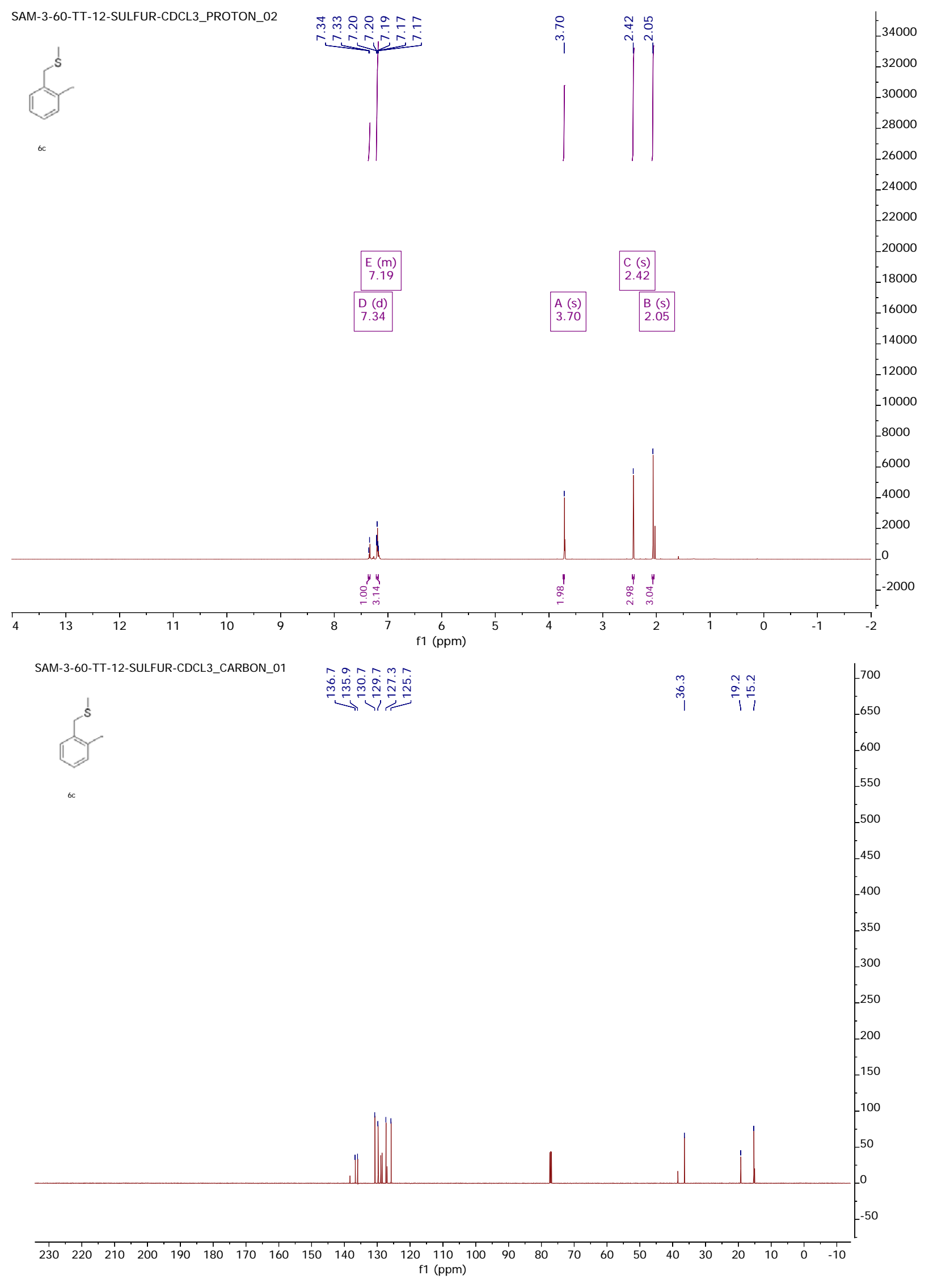




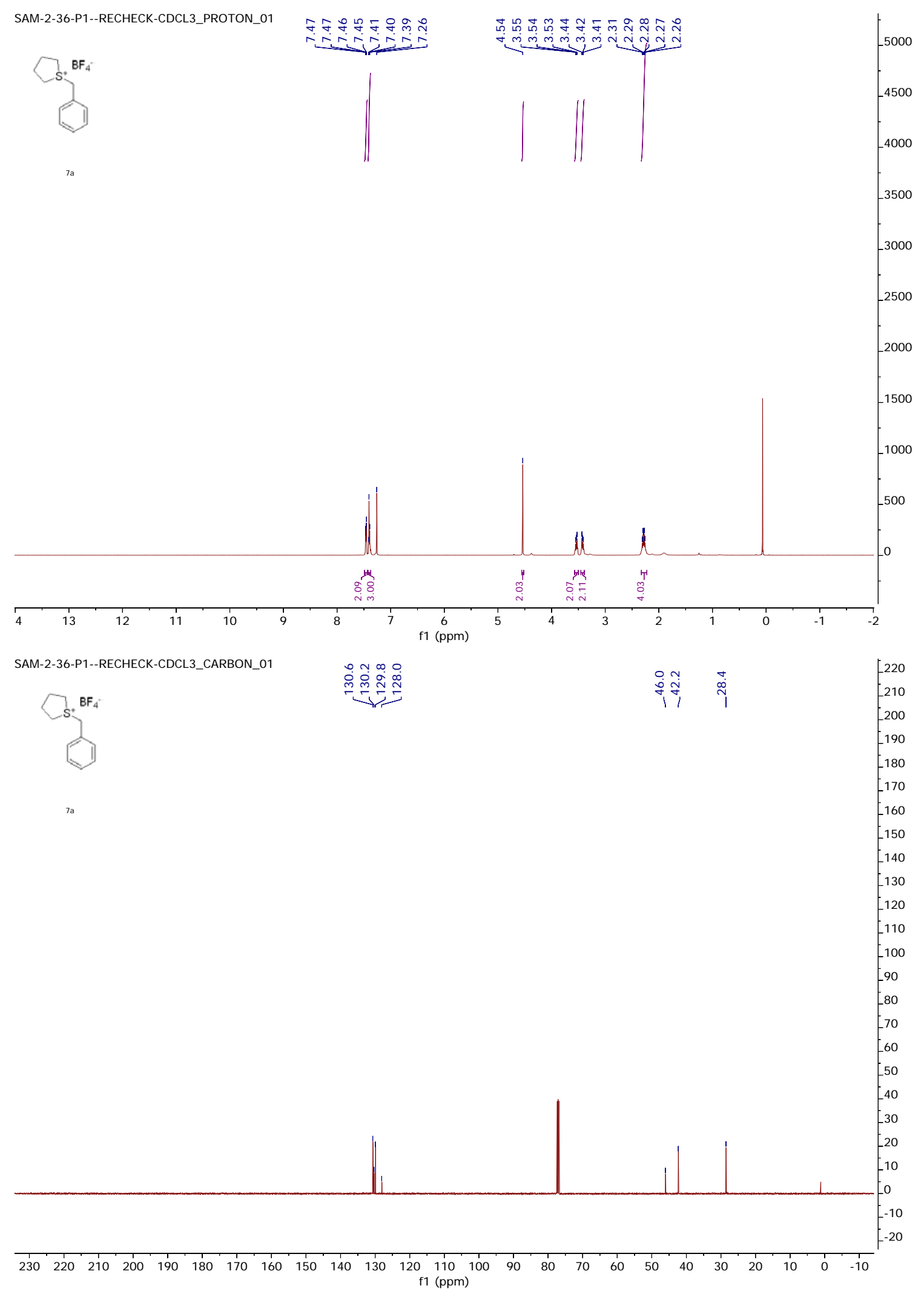




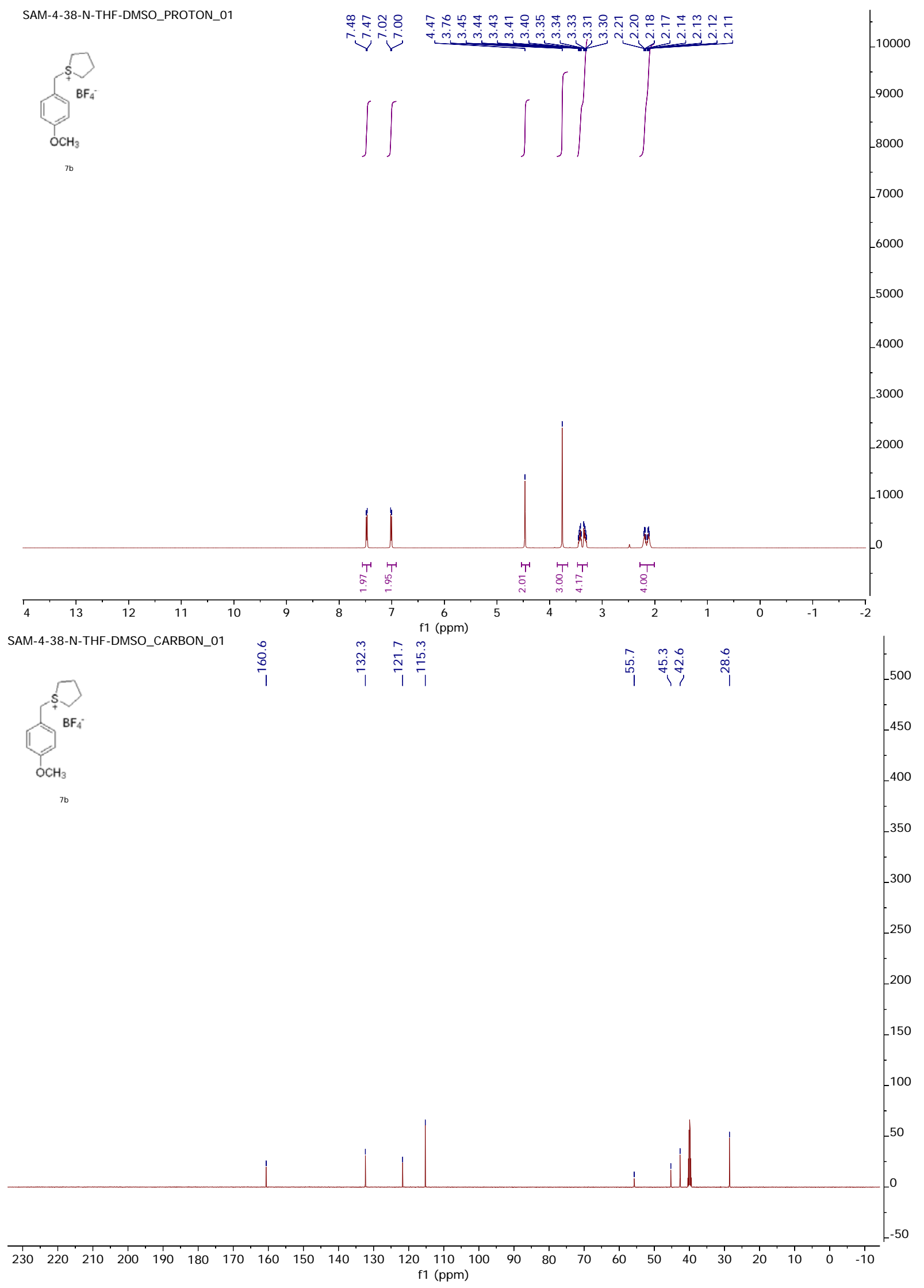




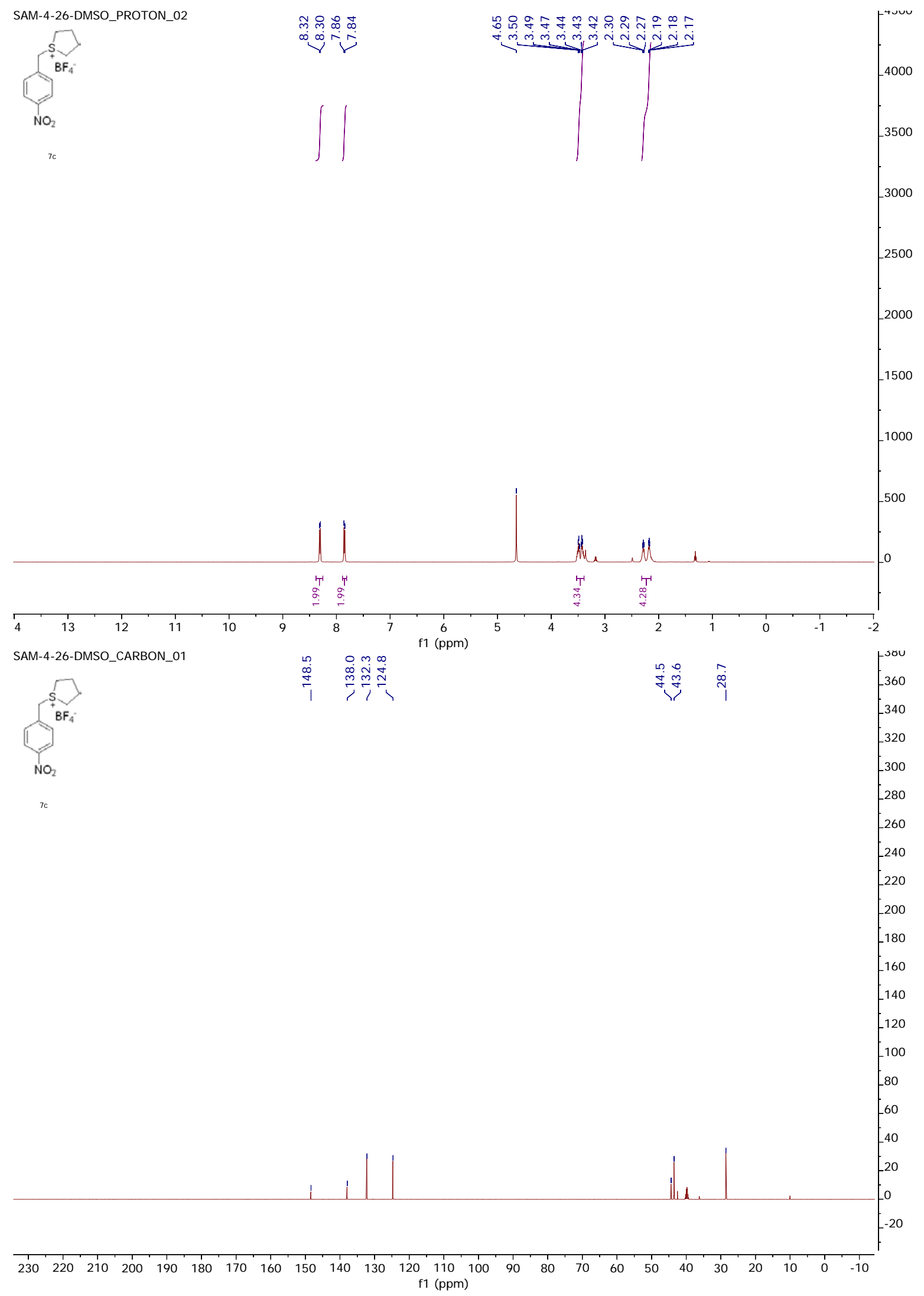

S61 


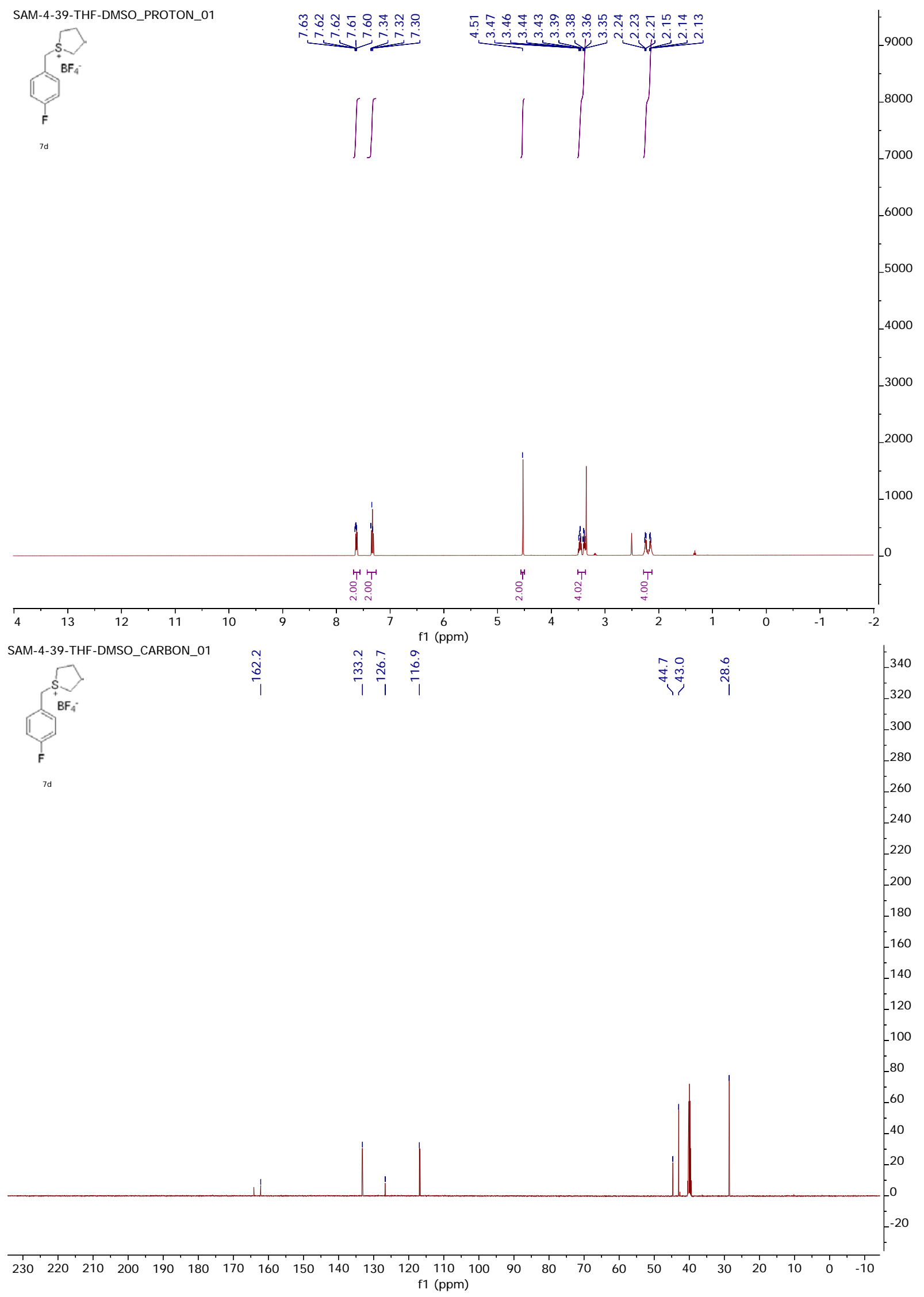




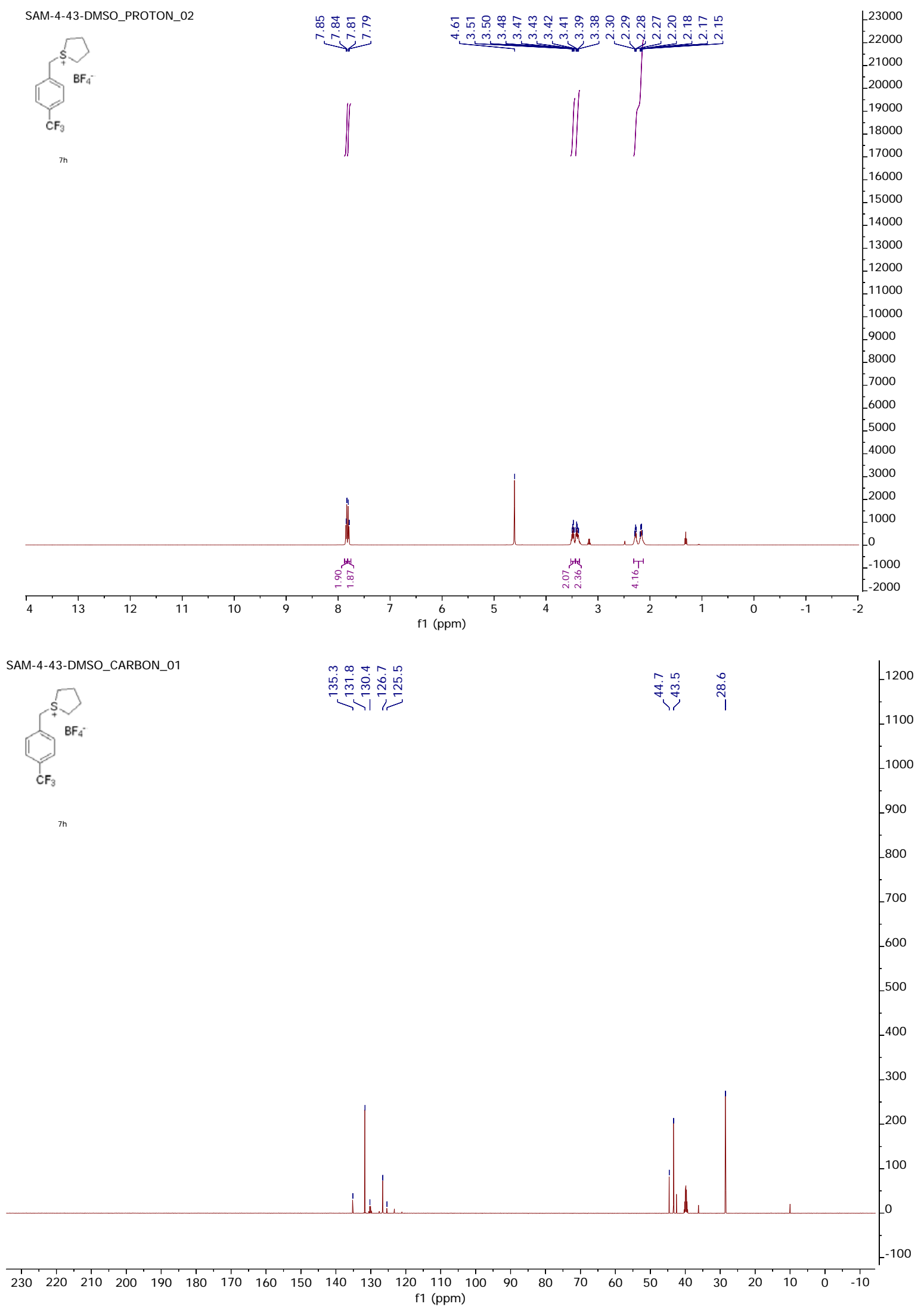




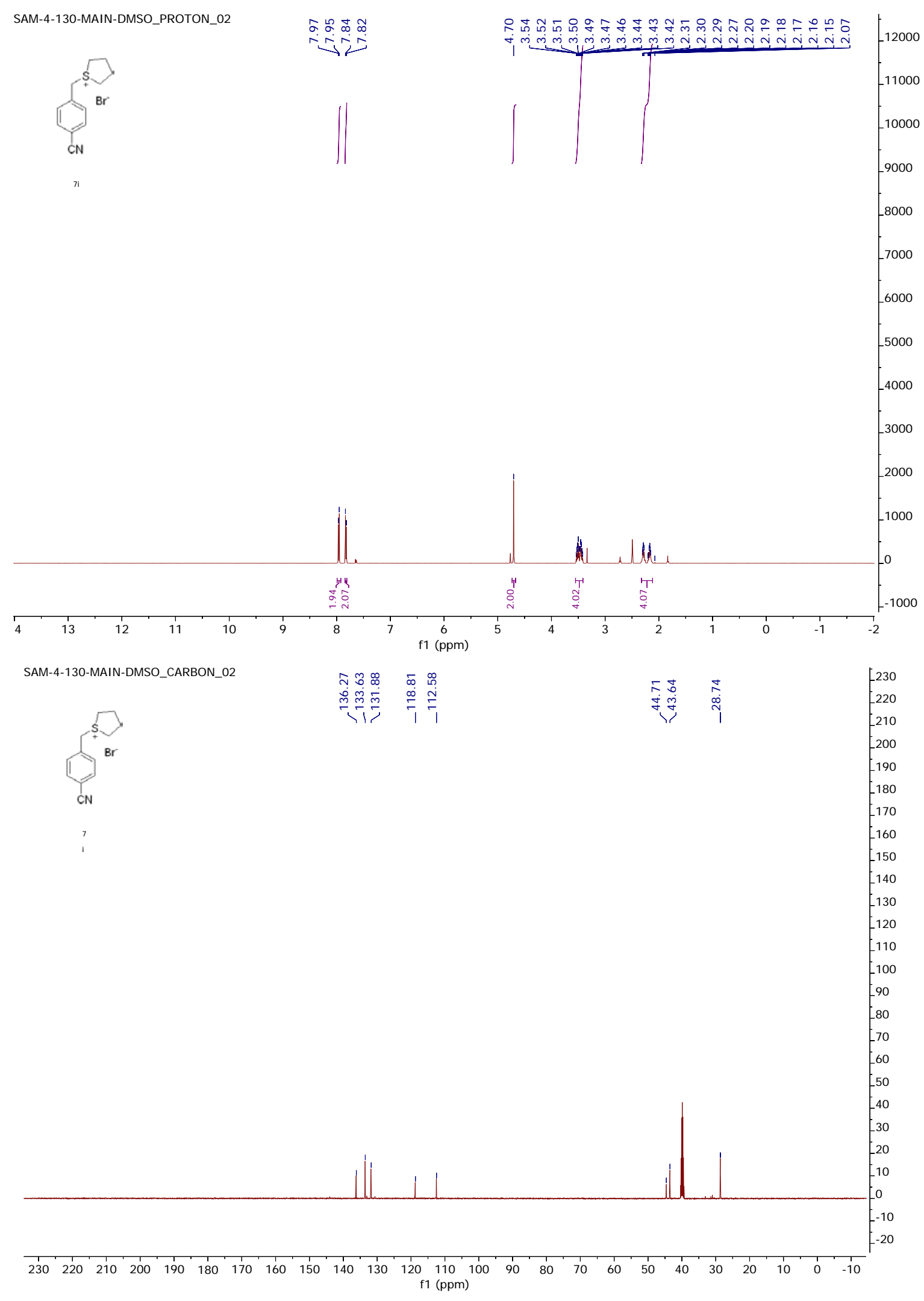




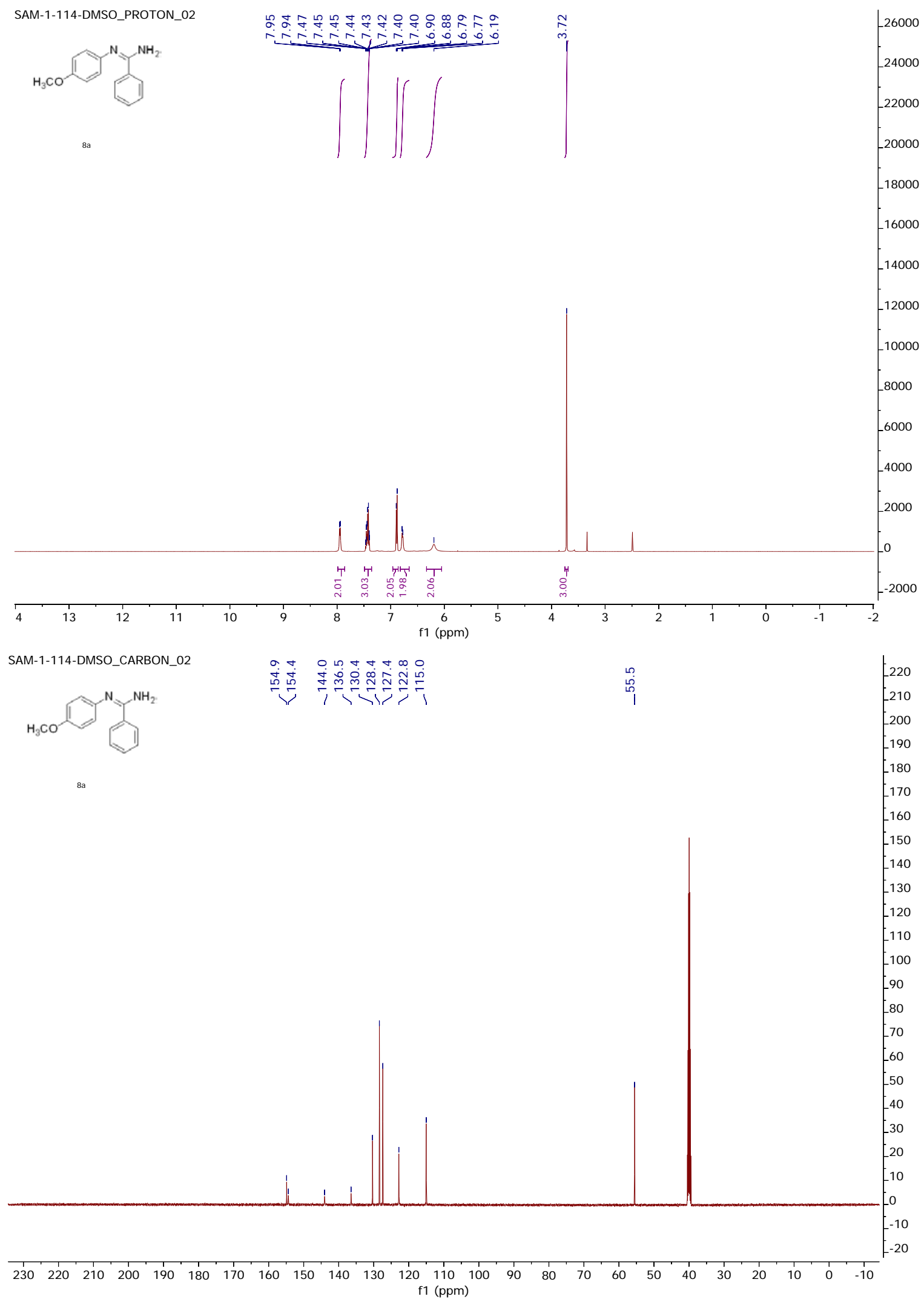




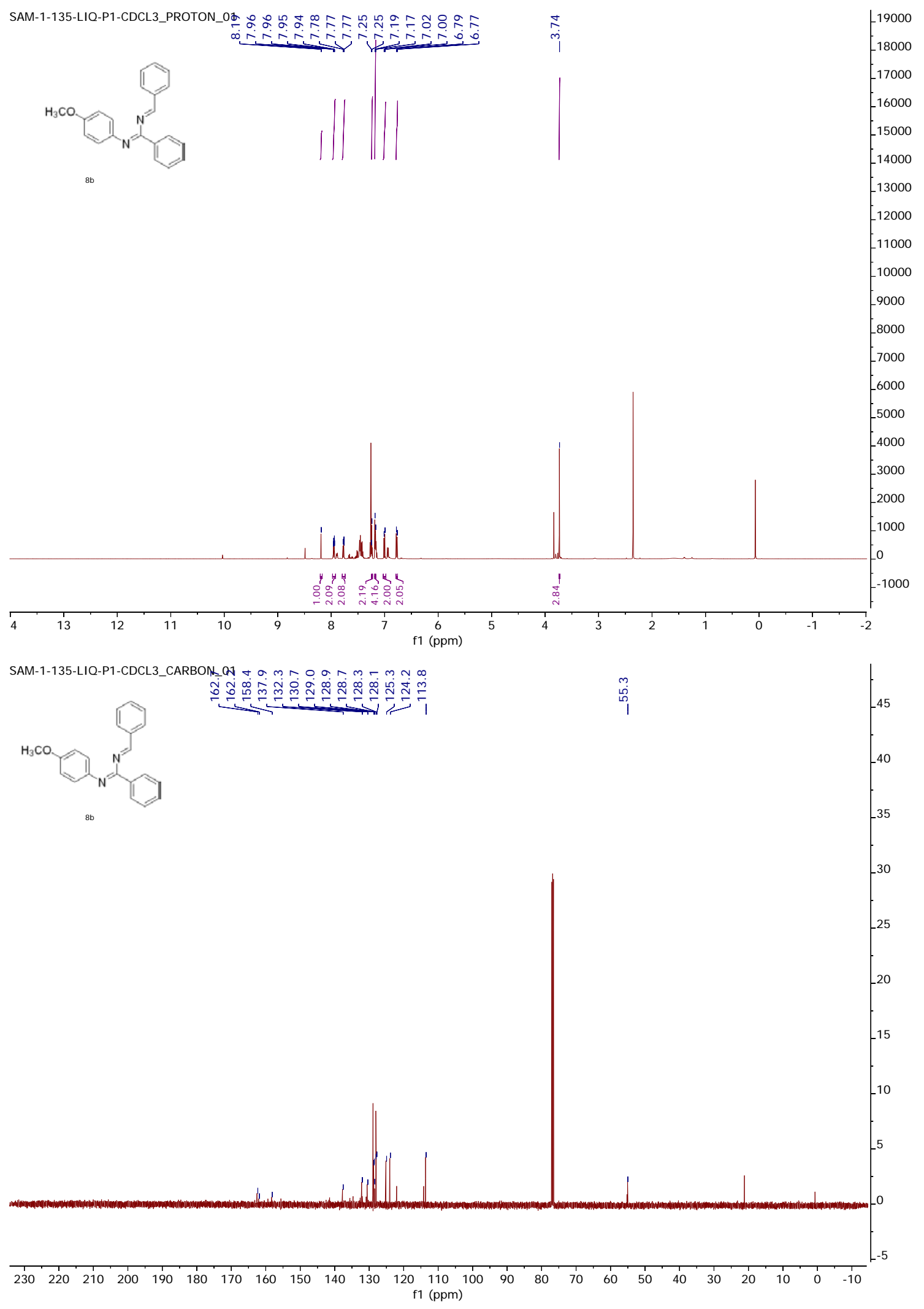



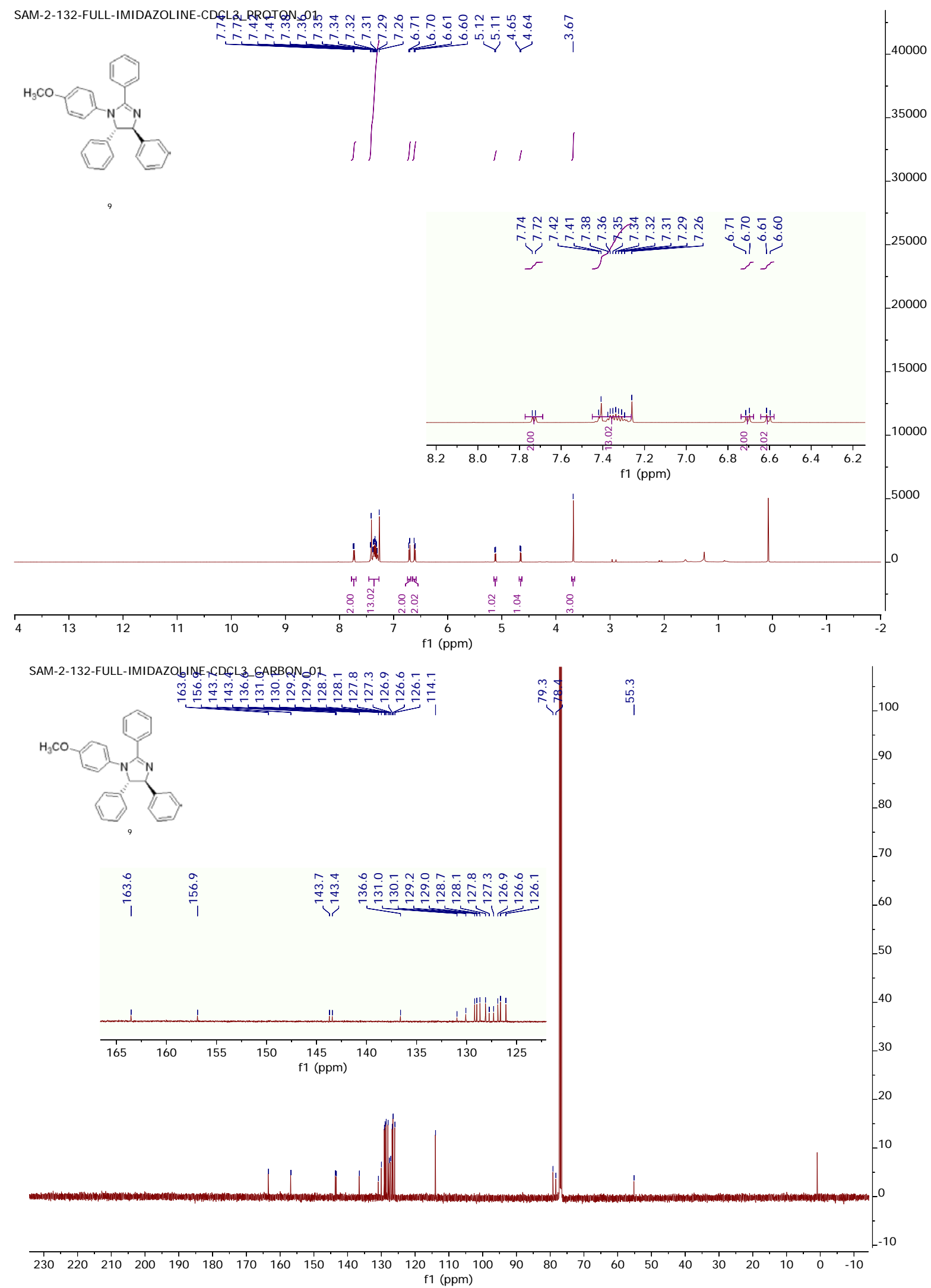